\title{
HIGH-DIMENSIONAL QUANTUM COMMUNICATION
}

\author{
Hoog-dimensionale \\ kwantumcommunicatie
}


Promotiecommissie

Voorzitter en secretaris

Promotor

Overige leden decaan TNW

Prof. Dr. P.W.H. Pinkse

Prof. Dr. Ir. J.W.M. Hilgenkamp

Prof. Dr. A. Lagendijk

Dr. C. Marquardt

Prof. Dr. D. Bouwmeester

Cover image: edited photograph of laser beams by Tom Wolterink, Ravitej Uppu, Yin Tao and Tristan Tentrup.

The work described in this thesis was carried out at the Complex Photonic Systems chair, Department of Science and Technology and MESA+ Institute for Nanotechnology, University of Twente, P.O. Box 217, 7500 AE Enschede, The Netherlands. 


\section{HIGH-DIMENSIONAL QUANTUM COMMUNICATION}

\section{PROEFSCHRIFT}

ter verkrijging van

de graad van doctor aan de Universiteit Twente, op gezag van de rector magnificus,

Prof. Dr. T.T.M. Palstra,

volgens besluit van het College voor Promoties

in het openbaar te verdedigen

op vrijdag 20 juli 2018 om 12.45 uur

door

\section{Tristan Bernhard Horst Tentrup}

geboren op 14 juni 1989

te Völklingen, Duitsland 
Dit proefschrift is goedgekeurd door de promotor Prof. Dr. P.W.H. Pinkse 


\section{Contents}

1 Introduction 1

1.1 History of Cryptography . . . . . . . . . . . . . . . . . 1

1.2 Large-alphabet quantum communication . . . . . . . . . . . . 3

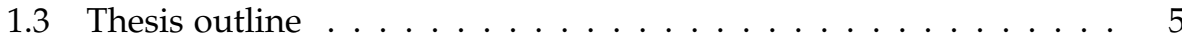

2 Concepts 7

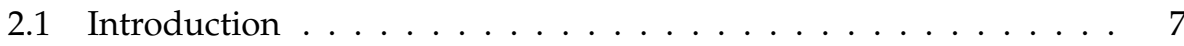

2.2 Shannon Entropy . . . . . . . . . . . . . . . . 7

2.3 Mutual Information . . . . . . . . . . . . . . . . . . . 8

2.4 Measurement operator . . . . . . . . . . . . . . . . . . 10

2.5 State discrimination . . . . . . . . . . . . . . . . . 10

2.6 Quantum information and Holevo bound . . . . . . . . . . . . . 13

2.7 Mutually unbiased bases . . . . . . . . . . . . . . . . . . 13

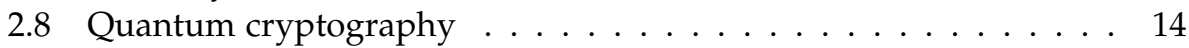

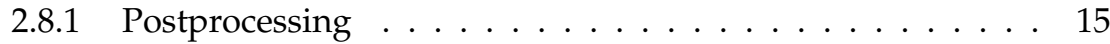

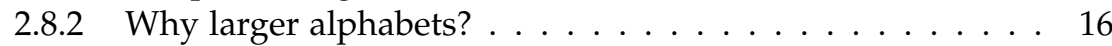

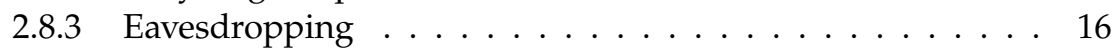

2.9 Encoding . . . . . . . . . . . . . . . . . . 19

2.10 Conclusion . . . . . . . . . . . . . . . . 21

3 Upper bound on the mutual information 23

3.1 Introduction . . . . . . . . . . . . . . . . . 23

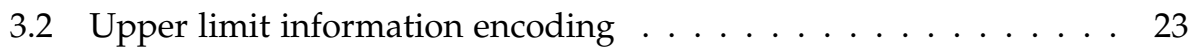

3.2.1 Upper limit including detector noise . . . . . . . . . . 26

3.2.2 Upper limit including channel noise . . . . . . . . . . . . . 29

3.3 Conclusion ...................... 31

4 Transmitting more than 10 bit with a single photon 33

4.1 Introduction . . . . . . . . . . . . . . . . 33

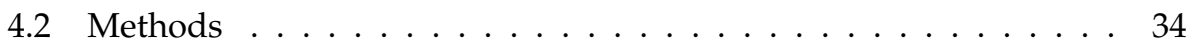

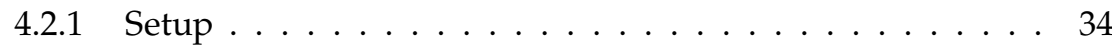

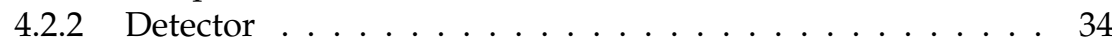

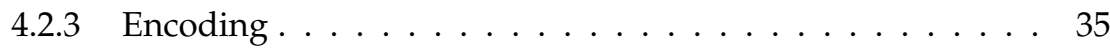

4.3 Result ...................... 36

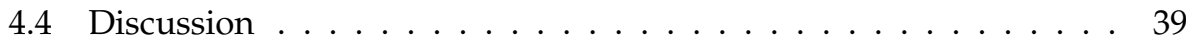

4.5 Conclusion ........................ 41

$5 \quad$ Large-Alphabet QKD 43

5.1 Introduction . . . . . . . . . . . . . . . . . . 43

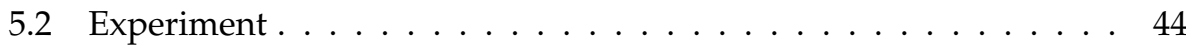

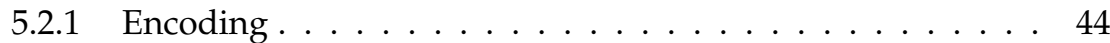

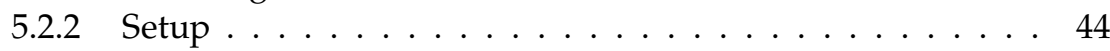


5.2 .3 Detection . . . . . . . . . . . . . . . 47

5.2.4 Classical light measurements . . . . . . . . . . . . . . . . 47

5.2 .5 Timing and Losses . . . . . . . . . . . . . . . . . 51

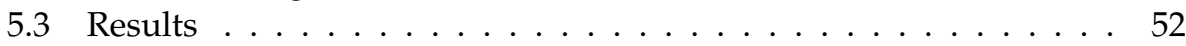

5.4 Discussion ............................ 54

5.4 .1 Intercept-resend attack . . . . . . . . . 56

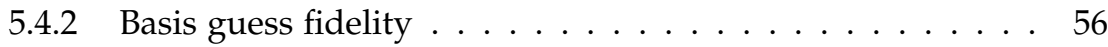

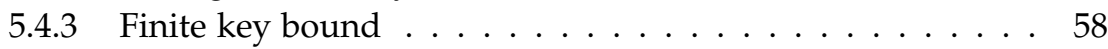

5.5 Conclusion ............................ 60

6 Multimode Fiber Communication 61

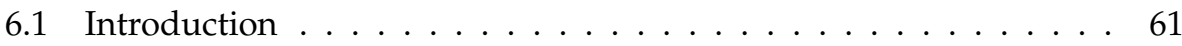

6.2 Result . . . . . . . . . . . . . . . . . 62

6.2.1 Experimental demonstration of secure communication . . . 63

6.2.2 Security with a single-photon light source . . . . . . . . . 67

6.2.3 Security with a weak coherent light source . . . . . . . . . 67

6.3 Methods . . . . . . . . . . . . . . . . . 69

6.3 .1 Experimental setup . . . . . . . . . . . . 69

6.3.2 Calibration and wavefront shaping procedure . . . . . . 71

6.3 .3 Image processing . . . . . . . . . . . . . 72

6.3.4 Information gained by Bob ............ 72

6.3 .5 Attacker model . . . . . . . . . . . . . 73

6.3.6 Security analysis for a single-photon Fock state . . . . . . . 74

6.3.7 Security analysis for coherent state - intensity measurement 76

6.3.8 Security analysis for coherent state - field measurement, unknown basis . . . . . . . . . . . . 76

6.3.9 Security analysis for coherent state - field measurement, known basis . . . . . . . . . . . . . . . 77

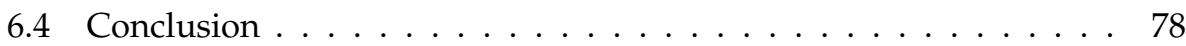

7 Comparison of Quantum Communication Methods 81

7.1 Introduction . . . . . . . . . . . . . . . . 81

7.2 Quantum-Secure Authentication . . . . . . . . . . . . . 81

7.3 Quantum Data Locking . . . . . . . . . . . . . . . . . 83

7.4 QSA in terms of QDL . . . . . . . . . . . . . . . 84

7.5 PEAC and MMFC . . . . . . . . . . . . . . . 86

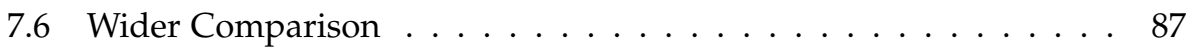

7.7 Conclusion . . . . . . . . . . . . . . . . 88

8 Summary and Outlook 89

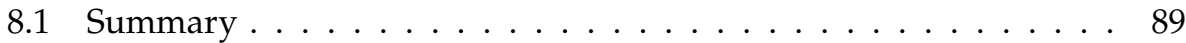

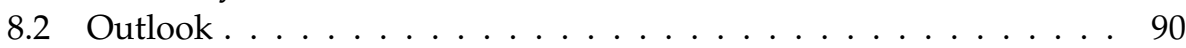


References

Samenvatting

105

Acknowledgements 



\section{Introduction}

\subsection{History of Cryptography}

From the early days of human civilization, certain written information needed to be kept secret in order to keep political, economical and military advantages. Even if an attacker captures the text, he shall not be able to extract its meaning. This motivated the development of cryptography.

The word cryptography originates from the Greek language, where kryptos translates into 'hidden' and graphia means 'writings'. Cryptography is the study of encryption, where encryption is the transformation of information such as plaintext with a key into a seemingly meaningless ciphertext. The inverse process is called decryption. One of the first cryptography methods has been used by the ancient Greeks [1]. The Spartan military used a device called a scytale, a wooden cylinder with a strip of leather wound around it, to encrypt their messages during battles. Both sender and receiver use a cylinder with the same diameter to encode and decode the message. The message is written on the leather lengthwise and the diameter of the cylinder is the key for encryption and decryption. Effectively, the text is rearranged in $x$ columns and read from top to bottom to generate the ciphertext. This is an example of a transposition cipher, where the encoding is done by permutations of the letters of the plaintext.

In the roman empire, the Caesar cipher was used for cryptography [1]. It was named after Julius Caesar, who used it in his private correspondence ${ }^{1}$. In the encryption, each letter of the plaintext is shifted $x$ positions in the alphabet to generate the ciphertext. The number $x$ of shifted positions is the key. An attacker can easily obtain $x$ either by brute force (only 23 shifts to test for the classical Latin alphabet) or by monitoring the distribution of letters of the ciphertext and comparing it to the distribution of letters in a typical text in this language. This characteristic distribution will be observed to be shifted by $x$ positions. To prevent this kind of analysis, the Vigenère cipher was developed $[1,2]$. Instead of shifting the message letters a fixed number of positions, the plaintext letters are shifted by a series of Caesar ciphers based on the letters of a keyword. For decryption and encryption, the Vigenère table, a table of shifted alphabets, was used. Although being rather simple, breaking this encryption took three centuries and was finally achieved by Friedrich Wilhelm Kasiski in 1863 [3].

The spread of telegraph networks raised the need for automatic cryptography machines. Gilbert Vernam patented using the XOR operation of a key tape on the plaintext to create the ciphertext, which can be read out with an identical key tape [4]. If this key tape contains random bits of the same length as the plaintext, the key is called a one-time pad and is proven to be secure [5]. The disadvantage is that one has to share as many key bits as there are message bits

1 Suetonius, Vita Divi Julii 56 
in advance to communicate.

To avoid the problem of heavy and large key tapes to encrypt and decrypt intense message traffic, especially in times of war, the rotor machine was independently developed by a number of inventors. Edward Hebern invented one of the first rotor machines in 1917 and patented the idea in 1921 [6]. A rotor machine is an electromechanical encryption and decryption device. It consists of at least one rotor and ring with electrical contacts, closing a wire connection between a key of a typewriter and a light bulb labeled with a letter. When a key is pressed, one bulb lights up. So far this would be a rather simple substitution of letters, which would easily be detected by frequency analysis. This is why after each key stroke, the rotor rotates one position. In this way, after 26 key strokes, the same substitution is used again. Adding $x$ rotors, the substitution repeats only every $26^{x}$ key strokes. One of the most famous rotor machines was the Enigma designed by Arthur Scherbius [7]. The Enigma additionally employed a plugboard, where the connections could be interchanged. The Enigma code was broken during World War II by the Turing bombe named after the British mathematician Alan Turing.

Claude Shannon started the field of information theory in 1948 [8] by introducing a measure of the uncertainty in a message, the information entropy. This had great influence on how information is encoded to transmit from a sender to a receiver. His main contribution to cryptography was realizing that all unbreakable ciphers need a one-time pad [5]. Cryptography based on one-time pads is "symmetric", meaning that the same key used for encryption needs to be used for decryption.

Ron Rivest, Adi Shamir and Leonard Adleman published one of the first public-key cryptosystems (RSA) in 1978 [9]. RSA is an asymmetric cryptography scheme consisting of two mathematically linked keys, a public key and a private key. As the name suggests, the private key needs to be kept secret. Either one of the keys can be used for encryption, where the other one will decrypt the message, therefore being asymmetric. The security relies on one-way functions, which are hard to invert, e.g., multiplication of two large prime numbers. The inverse process is factoring of large numbers, which is computationally hard. RSA is potentially threatened by quantum computers running Shor's algorithm [10].

One solution to this thread of quantum computers would be to generate a one-time pad between two parties by using concepts of quantum mechanics. The idea rose from the coin flipping problem [11]. In this problem two parties Alice and Bob don't trust each other, but still want to share the result of a coin toss via telephone. The new insight gained from this problem and Wiesner's work on quantum banknotes [12] had been used by Charles Bennett and Gilles Brassard to suggest the first Quantum Key Distribution (QKD) protocol BB84 [13] in 1984 after they met swimming at a beach [14] in 1979. Notably, 1983 is seen as the official birth year of QKD with the first description of BB84 in a presentation [15]. 
Its security is based on the no-cloning theorem [16], stating that an unknown quantum state can't be perfectly cloned. The first experimental realization of BB84 followed a few years later [17, 18]. Before that, the field didn't attract much attention. This changed when Artur Ekert proposed his protocol in 1991 [19]. In this protocol, instead of sending one qubit from a sender (Alice) to a receiver (Bob), two entangled photons from the same source are used, one qubit to Alice and the other one to Bob. It is based on the Einstein-Podolsky-Rosen thought experiment (EPR) [20] and involves Bell's theorem [21] to ensure security. At first this entanglement-based protocol experienced some resistance, since it was argued that any variant of BB84 could be adapted to use an entangled photon source and that Bell measurements would not be necessary [22]. Eventually this conflict was solved [23]. The use of entangled photon sources enriched the field of quantum cryptography and eventually led to device-independent QKD [24].

From there on many different protocols have been proposed and demonstrated [25-27]. As an extension of the BB84 protocol the full Hilbert space can be employed by introducing a third basis, resulting in the six-state protocol [28]. Due to the risk of photon number splitting attacks on weak coherent lasers as sources [29, 30], the SARG04 protocol [31] was proposed. The SARG04 protocol uses the same states and measuremants on Bob's side as the BB84 protocol. In contrast to BB84, the information is encoded in the basis choice of Alice, resulting in a more complicated sifting step of the protocol. The SARG04 protocol only requires a software change compared to BB84. Many other practical realizations have been demonstrated, including fiber-based $[32,33]$ and free-space line-ofsight satellite based [34-37] implementations. Some QKD systems are already commercially available [38] and in an ongoing race against quantum hacking $[39,40]$.

\subsection{Large-alphabet quantum communication}

The standard implementation of the BB84 protocol uses the two-dimensional polarization basis to encode information in photons. Therefore the alphabet contains only two symbols: "0" and "1", limiting the information content per photon to 1 bit. Especially in encrypted video communication [37] this key generation rate is the bottleneck. There are two approaches to increase the key generation rate. One is to increase the repetition rates of photon generation [41] and detection [42], which is inherently limited by dead times and jitter of the detectors [43]. The other approach is to exploit properties of photons besides the polarization to increase the dimensionality of the Hilbert space. A higher-dimensional Hilbert space leads to a higher information content of the photons and finally increases the key generation rate. Increasing the dimension of the basis using a large alphabet increases the information content per photon together with an improvement in the security $[26,44]$. 
This is why larger alphabets are employed [45-47] using orbital angular momentum [48-51], time binning [52-54] or spatial translation [55-58]. In a practical scenario, assuming a sender-receiver configuration with apertures of finite size, a diffraction-limited spot translated in space or Laguerre-Gauss modes have a higher capacity limit than the subset of pure OAM states $[59,60]$. This makes spatial positioning of light or equivalently, tilting of plane waves, an ideal method for increasing the information content per photon. Interestingly, given infinite resources, there is no known upper bound for the information content transmitted by single photons. For example, using one mole $\left(6.022 \cdot 10^{23}\right)$ of ideal position-sensitive single-photon detectors leads to an information content of 79 bit per detected photon. Clearly, this is out of reach in a practical situation. A very relevant question therefore is what can be realized experimentally. A partial answer to that is discussed in this thesis. With this high-dimensional encoding, we experimentally demonstrate very-high-dimensional two-bases BB84 QKD with 1024 distinguishable symbols in two mutually unbiased bases with a shared information of 7 bit per sifted photon.

There are regimes where one requires a higher-dimensional Hilbert space or a larger number of modes inherently for the quantum protocol. One example is Quantum Secure Authentication (QSA) [61]. In this protocol a multiplescattering medium, which is sufficiently complex and large that technology does not allow to copy it, serves as an optical Physical Unclonable Key [62]. To transmit high-dimensional wavefronts one needs a free line of sight or a multimode or a multicore optical fiber. It is therefore a highly relevant question to what extent multimode fibers are suitable for the transmission of complex wavefronts with many spatial dimensions. In this thesis, a new type of quantum communication method is experimentally demonstrated. This MultiMode Fiber Communication (MMFC) method is based on a high-dimensional spatial alphabet encoded into the guided modes of a multimode fiber by wavefont shaping. The multimode fiber combines features of a Physical Unclonable Function (PUF) [63, 64] with the inherent ability to generate secret keys by configuration changes. The experimental realization of the method provides security in a simple setup and real-life implementations, which makes commercial applications conceivable. 


\subsection{Thesis outline}

This thesis is about the further development of high-dimensional spatial-alphabet quantum communications. It extends the spatial dimension to the edge of technology, devises protocols for more secure methods and combines it with multimode fibers for long-distance applications.

Chapter 2 introduces the physical concepts used in this thesis. Important tools in classical information theory are explained and compared to quantum information theory. The challenge of quantum state discrimination is posed. The BB84 protocol is introduced together with classes of attack strategies. Finally, the spatial encoding scheme used in chapter 4 and 5 is introduced.

Chapter 3 gives analytic expressions for the upper bound on the mutual information transmitted between a sender and a receiver under various scenarios.

Chapter 4 presents the results of spatially encoding 10 bit of information in single photons.

Chapter 5 shows the results with a BB84-like large-alphabet QKD scheme with spatially encoded photons. A method to hide the basis choice of the sender is presented and discussed. The setup is characterized and the security of the protocol is analyzed.

Chapter 6 introduces MMFC, a novel secure multimode fiber communication protocol and analyzes its security.

Chapter 7 concludes the thesis by a comparison between quantum cryptography and quantum authentication protocols. They are analyzed in terms of Quantum Data Locking. Moreover, the Multimode Fiber Communication (MMFC) described in chapter 6 is compared to other protocols. 



\section{Concepts}

\subsection{Introduction}

Communication is the exchange of information by speaking, writing or via any other medium. In this thesis, information transfer between a single sender "Alice" and one receiver "Bob" is considered. Unfortunately, a third party "Eve" can eavesdrop on this communication. If Alice and Bob want to prevent Eve from reading their communication, they have to apply cryptography. Cryptography encompasses communication, where the sent information is not a plain, but an encrypted message. The encryption can be done by the use of a pre-shared key.

The most straightforward encoding is the one-time pad [4], where the message is encrypted by a random bit string acting as a key. A random bit string of at least the same length as the message bit string is combined with the message by XOR operation to encrypt or decrypt the message. This method is proven to be secure [5]. In this thesis, this random bit string is the key generated from the information exchanged between Alice and Bob. To allow the sending of longer messages, the bit string length needs to be maximized. Naturally, as a first step, the sent information needs to be increased.

A protocol to perform this task is presented in section 2.8. Before that, the information content of a message needs to be quantified. One method of quantification is the Shannon entropy, as introduced below.

\subsection{Shannon Entropy}

The Shannon entropy can be used as a measure for the average information content of an alphabet $X$ of discrete random variables $x$ with probability $P(x)$ and is defined as [8]

$$
H(X)=\sum_{x} P(x) \log _{2} \frac{1}{P(x)}
$$

where the Shannon information content of a particular $x$ of the ensemble is $\log _{2} 1 / P(x)$. This entropy is also called the uncertainty of $X$ and is historically defined by the logarithm with base 2 . Therefore, the Shannon information can be interpreted as the number of yes-no questions one has to answer to reveal the information. This uncertainty or entropy reaches its maximum for uniform probability distributions. An alphabet $X$ with non-uniform probability distribution has a smaller Shannon entropy.

As an example, consider the letter frequency in root words of a English dictionary ${ }^{1}$. In this example, the relative probability of each of the 26 letters can be counted. It turns out that the least frequent letter is the ' $z$ ' with a probability of $0.07 \%$ and a Shannon information of 10.4 bit. The most frequent letter ' $\mathrm{e}$ ' has

\footnotetext{
1 http://en.algoritmy.net/article/40379/Letter-frequency-English (15.04.2018)
} 
a probability of $12.7 \%$ and a Shannon information of only 3 bit. Receiving a ' $z$ ' contains more information due to its rareness. The overall ensemble has a Shannon entropy of $4.2 \mathrm{bit}$, which is lower than the one of the uniform distribution with a maximum of $\log _{2} 26=4.7$ bit. One might also say that english text is not the most efficient coding for information.

\subsection{Mutual Information}

This section introduces a measure for the transmitted information between two parties. In order to do so, new entropies need to be defined. The joint entropy of $X$ and $Y$ is

$$
H(X, Y)=\sum_{x, y} P(x, y) \log _{2} \frac{1}{P(x, y)},
$$

with the joint probability $P(x, y)$, the probability that $x$ was sent and $y$ received.

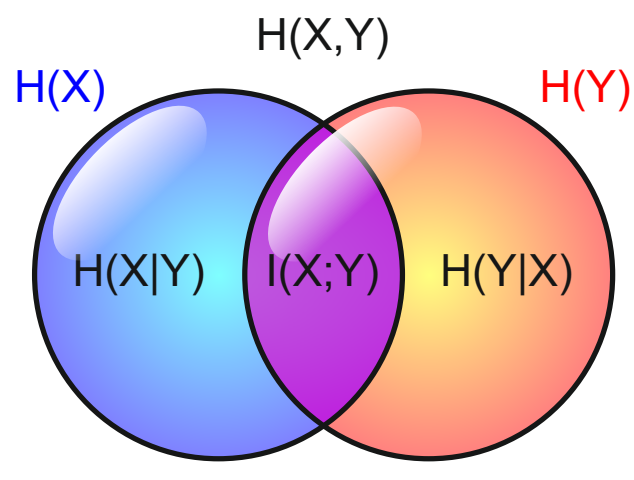

Figure 2.1 The relationship between the marginal entropies $H(X)$ (blue) and $H(Y)$ (red), the joint entropy $H(X, Y)$, conditional entropies $H(X \mid Y)$ and $H(Y \mid X)$ and the mutual entropy $I(X ; Y)$ (pink).

The information shared between a sender with entropy $H(X)$ and a receiver with entropy $H(Y)$ is called mutual information $I(X ; Y)$ and is illustrated in figure 2.1. It can be expressed in the following ways [65]:

$$
\begin{aligned}
I(X ; Y) & =H(X)+H(Y)-H(X, Y) \\
& =H(Y)-H(Y \mid X) \\
& =H(X)-H(X \mid Y),
\end{aligned}
$$


with the conditional entropy of $X$ given $Y$ defined as

$$
\begin{aligned}
H(X \mid Y) & =\sum_{y} P(y)\left[\sum_{x} P(x \mid y) \log _{2} \frac{1}{P(x \mid y)}\right] \\
& =\sum_{x, y} P(x, y) \log _{2} \frac{1}{P(x \mid y)},
\end{aligned}
$$

which is a measure for the average uncertainty about $x$ when $y$ is known. $P(x \mid y)$ is the conditional probability that $x$ is sent under the condition that $y$ is measured. Using Bayes' theorem

$$
P(x, y)=P(x) P(y \mid x)=P(y) P(x \mid y),
$$

equation (2.4) can be written as

$$
\begin{aligned}
H(X \mid Y) & =\sum_{x, y} P(x, y) \log _{2} \frac{P(y)}{P(y \mid x) P(x)} \\
& =\sum_{x, y} P(x, y) \log _{2} \frac{1}{P(x)}+\sum_{x, y} P(x, y) \log _{2} \frac{P(y)}{P(y \mid x)} .
\end{aligned}
$$

The summation over $y$ in the first term can be carried out to receive the entropy of the sent alphabet $H(X)$. Using again Bayes' theorem and inserting equation (2.6) into equation (2.3), the mutual information can be expressed as

$$
I(X ; Y)=\sum_{x, y} P(x, y) \log _{2} \frac{P(x, y)}{P(x) P(y)} .
$$

If one symbol out of a $d$-dimensional alphabet is received with probability $F$ and all other wrong symbols are measured with the same probability, we have for the joint probabilities to find $x$ and $y$

$$
\begin{array}{ll}
P(x, y)=\frac{F}{d} & \forall x=y \\
P(x, y)=\frac{1-F}{d(d-1)} & \forall x \neq y .
\end{array}
$$

Assuming all symbols are sent and received with the same probability

$$
P(x)=\sum_{y} P(x, y)=\frac{1}{d}=P(y),
$$

this simplifies equation (2.7) to

$$
I(X ; Y)=\log _{2}(d)+F \log _{2}(F)+(1-F) \log _{2}\left(\frac{1-F}{d-1}\right) .
$$




\subsection{Measurement operator}

So far, we have worked with classical information theory. To understand the influence of quantum mechanics in information theory, it is important to consider strategies to measure quantum states [27, 66]. A quantum measurement is described by a set of measurement operators $\left\{M_{m}\right\}$, which fulfill the completeness equation

$$
\sum_{m} M_{m}^{\dagger} M_{m}=1
$$

For a state $|\psi\rangle$, the $m$ measurement outcomes occur with probabilities

$$
p(m)=\left\langle\psi\left|M_{m}^{\dagger} M_{m}\right| \psi\right\rangle
$$

and the measurements put the system into the state

$$
\frac{M_{m}|\psi\rangle}{\sqrt{p(m)}} .
$$

The operator $E_{m}=M_{m}^{\dagger} M_{m}$ is called a Positive Operator-Valued Measure (POVM). POVMs are Hermitian and have positive expectation values $\left\langle\psi\left|E_{m}\right| \psi\right\rangle \geq 0$. If the measurement operators are Hermitian and satisfy $M_{m} M_{m^{\prime}}=\delta_{m, m^{\prime}} M_{m}$, one calls the measurement a projective measurement. Projective measurements are the most common measurements in quantum optics. The name of this measurement is a sign of the fact that a quantum state is projected onto the state associated to the projection in contrast to POVMs, where the state after the measurement is not determined. As a consequence, repeated sequential measurements with the same operator $M_{m}$ will lead to the same measurement result. The measurements performed in the experimental chapters are mostly projective measurements. In this context, a measurement in basis $|m\rangle$ is a projective measurement with operators $P_{m}=|m\rangle\langle m|$ in the orthonormal basis $|m\rangle$.

\subsection{State discrimination}

The simplest example of state discrimination is the discrimination between two qubits. A qubit is a two-dimensional quantum state $a|0\rangle+b|1\rangle \quad\left(a, b \in \mathbb{C},|a|^{2}+\right.$ $|b|^{2}=1$ ) on the orthonormal basis $|0\rangle,|1\rangle$. The best what Bob can hope for is to distinguish two states, which is only guaranteed to work for orthogonal states. For example, Alice prepares the two states $\left|\psi_{1}\right\rangle=|1\rangle$ and $\left|\psi_{2}\right\rangle=(|0\rangle+|1\rangle) / \sqrt{2}$ with equal probability and sends them to Bob, who has to decide which state he received. The optimal minimum error strategy based on projective measurements is to define two orthogonal states $\left|\phi_{1}\right\rangle$ and $\left|\phi_{2}\right\rangle$ symmetric to the angular bisector of $\left|\psi_{1}\right\rangle$ and $\left|\psi_{2}\right\rangle$, where $\left|\phi_{1}\right\rangle$ has a larger overlap with $\left|\psi_{1}\right\rangle$. The projection is done with the operators $\left|\phi_{1}\right\rangle\left\langle\phi_{1}\right|$ and $\left|\phi_{2}\right\rangle\left\langle\phi_{2}\right|$. If the system is projected 
to $\left|\phi_{1}\right\rangle$, we guess $\left|\psi_{1}\right\rangle$ and vice versa. The probability of success is

$$
P_{\text {guess }}=\frac{1}{2}+\frac{1}{2}\left(1-\left|\left\langle\psi_{1} \mid \psi_{2}\right\rangle\right|^{2}\right)^{\frac{1}{2}}=\frac{1+\sqrt{2}}{2 \sqrt{2}} \approx 0.85 .
$$

Obviously, by guessing one will make errors in distinguishing the state. However, there is a strategy which allows to distinguish both states sometimes without giving a wrong answer, when also allowing an inconclusive result. This strategy is called unambiguous state discrimination [67-70]. The first scenario is that Bob performs projective measurements on the orthogonal states $\left|\psi_{1}^{T}\right\rangle$ and $\left|\psi_{2}^{T}\right\rangle$ as shown in figure 2.2 .

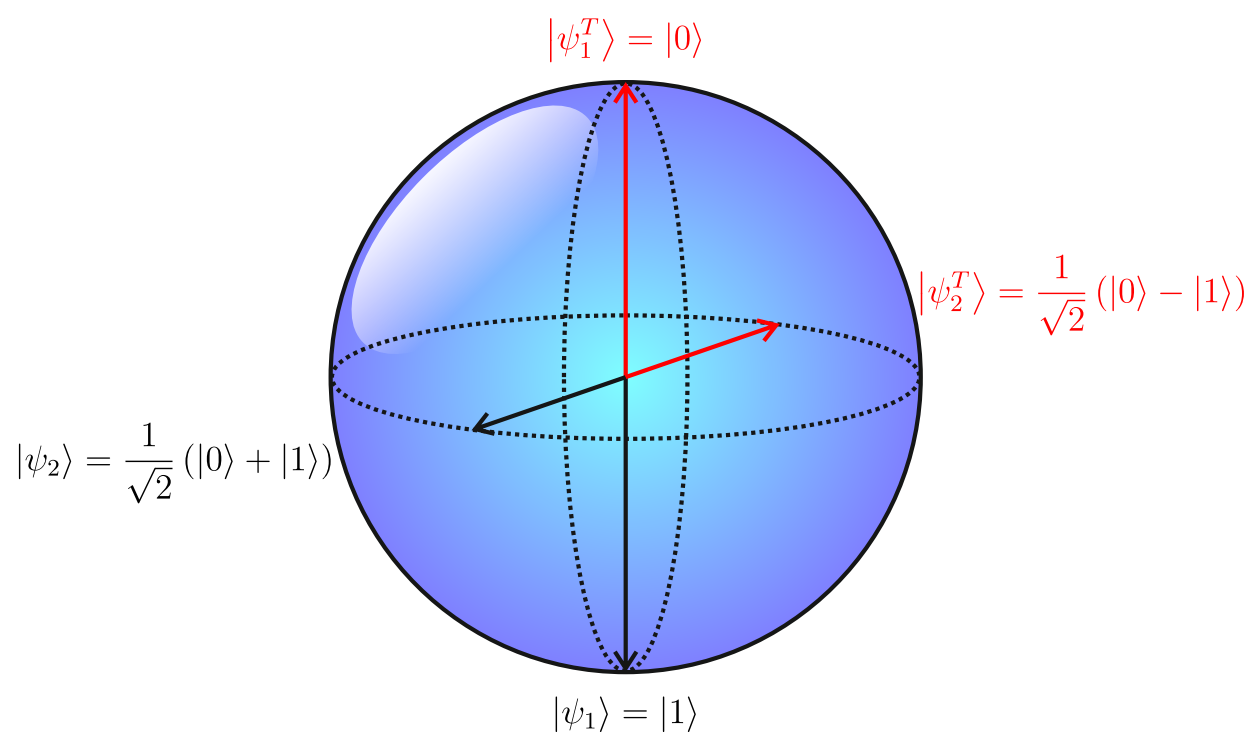

Figure 2.2 Illustration of the Bloch sphere with the two qubits $\left|\psi_{1}\right\rangle$ and $\left|\psi_{2}\right\rangle$ (black) and their orthogonal states $\left|\psi_{1}^{T}\right\rangle$ and $\left|\psi_{2}^{T}\right\rangle$ (red).

Half the time, his measurement operators are $\left|\psi_{1}\right\rangle\left\langle\psi_{1}\right|$ and $\left|\psi_{1}^{T}\right\rangle\left\langle\psi_{1}^{T}\right|$ (basis 1) and half the time $\left|\psi_{2}\right\rangle\left\langle\psi_{2}\right|$ and $\left|\psi_{2}^{T}\right\rangle\left\langle\psi_{2}^{T}\right|$ (basis 2). Suppose Bob performs a projective measurement in basis 1 . If the system after the measurement is projected to $\left|\psi_{1}^{T}\right\rangle$, he knows with certainty that the state was $\left|\psi_{2}\right\rangle$, since only $\left|\psi_{2}\right\rangle$ has a component in $\left|\psi_{1}^{T}\right\rangle$ direction. If the system after the measurement is projected to $\left|\psi_{1}\right\rangle$ on the other hand, the result is inconclusive, since beside $\left|\psi_{1}\right\rangle$ also $\left|\psi_{2}\right\rangle$ has a component in $\left|\psi_{1}\right\rangle$ direction. Similar arguments hold for measurement basis 2, noting that the two projective measurements act in two mutually unbiased bases (see section 2.7). The probability for a conclusive and 
correct result is

$$
P_{\text {proj }}=\frac{1-\left|\left\langle\psi_{1} \mid \psi_{2}\right\rangle\right|^{2}}{2}=\frac{1}{4}
$$

However, there is a strategy which allows to distinguish both states with higher probability using POVM operators. In this example, the POVMs are

$$
\begin{aligned}
& E_{1}=\frac{\mathbb{1}-\left|\psi_{1}\right\rangle\left\langle\psi_{1}\right|}{1+\left|\left\langle\psi_{1} \mid \psi_{2}\right\rangle\right|}=\frac{1}{1+\frac{1}{\sqrt{2}}}|0\rangle\langle 0| \\
& E_{2}=\frac{\mathbb{1}-\left|\psi_{2}\right\rangle\left\langle\psi_{2}\right|}{1+\left|\left\langle\psi_{1} \mid \psi_{2}\right\rangle\right|}=\frac{1}{1+\frac{1}{\sqrt{2}}} \frac{(|0\rangle-|1\rangle)(\langle 0|-\langle 1|)}{2} \\
& E_{3}=\mathbb{1}-E_{1}-E_{2}=\frac{1}{1+\frac{1}{\sqrt{2}}} \frac{|1\rangle\langle 1|-| 0\rangle\langle 0|+| 0\rangle\langle 1|+| 1\rangle\langle 0|}{2} .
\end{aligned}
$$

They fulfill all criteria for POVMS (see section 2.4). If Bob performs this set of measurements on state $\left|\psi_{1}\right\rangle$, he will never get the measurement outcome $E_{1}$, since $\left\langle\psi_{1}\left|E_{1}\right| \psi_{1}\right\rangle=0$. That means that the measurement result $E_{1}$ tells Bob with certainty that he has the state $\left|\psi_{2}\right\rangle$. A similar argumentation holds for $\left|\psi_{2}\right\rangle$. Only the measurement result $E_{3}$ does not tell Bob anything about the state; Bob would have to give the honest answer "I don't know". Nevertheless, the beauty of this method lies in the fact that Bob does not make a mistake in distinguishing the states, the method is error free. The cost is to allow an inconclusive result. The success probability of this unambiguous state discrimination is

$$
P_{\mathrm{POVM}}=1-\left|\left\langle\psi_{1} \mid \psi_{2}\right\rangle\right| \geq P_{\text {proj }}
$$

It can be generalized to $d$-dimensional states [71], where the construction of the set of POVMs is an optimization problem. For some special cases like symmetric states, this construction is known [72]. Another special case is formed by orthogonal states. Although a POVM measure leads to higher success probabilities for general states, in this case a projective measurement onto the states themself has already a unit success probability.

The optimal fidelity for state estimation of $N$ copies of a $d$-dimensional quantum system is [73]

$$
F=\frac{N+1}{N+d}
$$

That means a state can be estimated with a fidelity as close to unity as wanted, given enough copies. It would also mean that all states can be distinguished and that the Holevo bound is violated. The missing part in this dilemma is the no-cloning theorem [16]. The no-cloning theorem states that it is impossible to create an identical copy of an arbitrary unknown quantum state. 


\subsection{Quantum information and Holevo bound}

Section 2.3 includes a measure of the shared information between Alice and Bob. In contrast to the case with two classical states, a quantum measurement is not always able to distinguish between quantum states (see section 2.5). The amount of information that can be known about a quantum system is called the accessible information [27]. This accessible information is a measure for the shared information between Alice and Bob in case they communicate with quantum states. Perhaps surprisingly, there is no known formula to compute the accessible information. However, there are upper bounds such as the Holevo bound which gives the following inequality.

Suppose Alice prepares a possibly mixed state with density operator $\rho_{x}$ where $X=1, \ldots, d$ with probabilities $p_{1}, \ldots, p_{d}$. Bob performs a measurement described by POVM elements $\left\{E_{y}\right\}=\left\{E_{1}, \ldots, E_{m}\right\}$ on that state, with measurement outcome $Y$. For any such measurement, the Holevo bound states that

$$
I(X ; Y) \leq S(\rho)-\sum_{x} p_{x} S\left(\rho_{x}\right)
$$

where $\rho=\sum_{x} p_{x} \rho_{x}$ and $S(\rho)=-\operatorname{Tr}\left(\rho \log _{2}(\rho)\right)$ is the von Neumann entropy. The maximum accessible information is reached for the maximally mixed states $\rho=\sum_{n} 1 / d|n\rangle\langle n|$, with orthonormal basis $\{|n\rangle\}$, spanning a $d$-dimensional space with equal send probability $p_{1}, \ldots, p_{d}=\frac{1}{d}$, namely $\log _{2}(d)$ bit [74]. This result is surprising: even though Alice can prepare an infinite amount of information by an arbitrary superposition of just two of the $d$ quantum states $\rho_{x}$, Bob is only able to access $\log _{2}(d)$ bit of information from the set $\rho_{x}$. Hence, in the regime of orthogonal quantum states, quantum information is equivalent to classical information. At this point, one may wonder why quantum states are still interesting for communication. The answer is that the fact that the accessible information can be bound to be smaller than the prepared information enables a whole field of interesting applications in cryptography.

\subsection{Mutually unbiased bases}

Two orthonormal bases $\left\{\left|a_{1}\right\rangle, \ldots,\left|a_{d}\right\rangle\right\}$ and $\left\{\left|b_{1}\right\rangle, \ldots,\left|b_{d}\right\rangle\right\}$ are called mutually unbiased if

$$
\left|\left\langle a_{i} \mid b_{j}\right\rangle\right|^{2}=\frac{1}{d} \quad \forall i, j \in\{1, \ldots, d\} .
$$

Hence, a basis vector from the first basis is non-orthogonal with any basis vector from the second basis. If a quantum state is prepared in a state belonging to one of these bases, a measurement in the mutually unbiased basis will return all outcomes with equal probability. With other words: Nothing can be learned from a measurement in this basis. This makes them interesting for quantum cryptography. 
In general, it is an open question to find the number of mutually unbiased bases for a given dimension $d$. An exception are bases where the dimension is an integer power of a prime number. For them, it is known that $d+1$ bases can be found [75]. Unfortunately, there is no straightforward way to construct this maximum of $d+1$ bases for any $d$. On the other hand, one mutually unbiased basis can be always generated for any $d$ by Fourier transform. For the bases $\left\{\left|a_{1}\right\rangle, \ldots,\left|a_{d}\right\rangle\right\}$, this Fourier transformed basis is constructed by

$$
\left|b_{k}\right\rangle=\frac{1}{\sqrt{d}} \sum_{n=0}^{d-1}\left(\exp \left(\frac{2 i \pi}{d}\right)\right)^{k n}\left|a_{n}\right\rangle .
$$

As an example for $d=2$, the eigenvectors of the three Pauli matrices

$$
\sigma_{x}=\left[\begin{array}{ll}
0 & 1 \\
1 & 0
\end{array}\right], \quad \sigma_{y}=\left[\begin{array}{cc}
0 & -i \\
i & 0
\end{array}\right], \quad \sigma_{z}=\left[\begin{array}{cc}
1 & 0 \\
0 & -1
\end{array}\right]
$$

form a set of mutually unbiased bases. In the next section, these three bases are used for the best known quantum cryptography protocols.

\subsection{Quantum cryptography}

One way to exploit the quantum nature of light to generate a key between Alice and Bob is Quantum Key Distribution (QKD) [25, 26]. The first QKD protocol was published by Bennett and Brassard in 1984 [13]. Alice has a single-photon source. She encodes one bit of information "0" or "1" in the two-dimensional polarization basis of these photons. She encodes in the horizontal or vertical basis (see $\sigma_{z}$ in equation (2.25)) or the diagonal or anti-diagonal basis (see $\sigma_{x}$ in equation (2.25)). As apparent from the last section 2.7, these two bases are mutually unbiased. Alice and Bob can switch between the two bases by rotating their Polarizing Beam Splitter (PBS) by $45^{\circ}$. They align their PBS with respect to each other and agree on the encoding of the qubits. They possess an authenticated classical channel for postprocessing. They then perform the following steps:

1. Alice encodes a photon in one of the four states, sends it to Bob via the quantum channel and saves the sent state. Bob measures randomly in either the $\sigma_{z}$ or $\sigma_{x}$ basis. He records his measurement result and basis choice. Repeated $N$ times, they both have a list of measurement results and recorded bases.

2. Alice and Bob share their basis choices over a classical communication channel. They only keep results of measurements performed in the same basis. The rest of the key does not contain any information due to having been measured in the mutually unbiased basis. Thus, these measurements are discarded. After this so called sifting, Alice and Bob are left with $N / 2$ bits of raw key. 
3. If Eve had interaction with the quantum channel, the error rate must have increased. In order to estimate this error rate and thus the amount of information the eavesdropper extracted, Alice and Bob reveal a random sample $m$ of the raw key. In the error-free case, the remaining $n=N / 2-m$ bits are the secret key. If errors occurred, these errors need to be corrected and any information leakage to an eavesdropper needs to be erased [76]. This is done in postprocessing over the public channel. If Eve's information is larger than the one between Alice and Bob, no secret key can be generated.

This is a $d=2$ protocol. As seen in section 2.7 , there are three mutually unbiased bases for two dimensions. The third basis $\sigma_{y}$ is used in the six-state-protocol [28].

\subsubsection{Postprocessing}

Due to technical imperfections and the possible intervention of an eavesdropper, the sifted key has usual a Quantum Bit Error Rate (QBER) of a few percent. Consequently, the first step of postprocessing is to perform error correction or information reconciliation via the classical channel to reduce the Bit Error Rate (BER). One differentiates between one-way postprocessing, where either only Alice or Bob sends via the classical channel and two-way communication. The maximum amount of information which can be extracted by error correction is the mutual information. This limit is well known in noisy-channel coding as the Shannon limit [78]. The most efficient error-correction codes are two-way communication codes, which can be mapped to one-way communication [79]. One of the best studied error correction codes is the cascade code [80, 81]. The Cascade code is based on block parity exchange. In the first step, the bits of the sifted key are shuffled and divided in blocks of equal length. The initial block size is chosen as a function of the QBER. The sum modulo 2 of each block is calculated to compare the parity of the blocks. The parity values of all blocks are communicated via the two-way noiseless classical channel between Alice and Bob. In this way, all blocks with different parity can be found. In order to find the error, a divide and conquer algorithm is applied and the detected error can be corrected. The first pass of the error correction is done. Since even number of errors in a block will remain unnoticed and only a single error per block is corrected, this algorithm needs to run iteratively for a number of passes. In each pass the key is shuffled and the block size is doubled. If an error remains undetected after the first pass, this information can be used to uncover an odd number of additional errors in the succeeding passes. The algorithm can step back and correct also these errors. Sometimes this uncovers another error in a different pass, starting a cascade of corrections.

The second step of post processing is privacy amplification [76, 77, 82, 83]. The goal of privacy amplification is to erase Eve's knowledge of the sifted key and also the information she gained from error correction by sacrificing a part 
of the key. As a simple example, consider the case where Alice and Bob share two random bits. Eve knows the value of both bits with probability $\epsilon$ based on her attack strategy. Alice and Bob perform an XOR operation on the two bits bisecting their key to one bit. On the other hand Eve's success probability to guess this one bit is only $\epsilon^{2}+(1-\epsilon)^{2}$. In general privacy amplification is done using a universal family of hash functions [84]. To sum up the postprocessing is a purely classical task. In order to perform it efficiently (erasing Eve's knowledge by still maintaining a large key) the error correction and privacy amplification parameters have to be chosen carefully.

\subsubsection{Why larger alphabets?}

In the original BB84 protocol an alphabet with only two symbols 0 and 1 with a maximum of one bit encoded in a single photon is used. If instead of the 2-dimensional Hilbert space the encoding is done in a $d$-dimensional Hilbert space, the maximum information is $\log _{2}(d)$. A second non-orthogonal mutually unbiased basis set can be constructed by Fourier transform. This $d$-dimensional two-bases BB84 protocol shares the same steps as the original BB84 protocol. The difference is a larger information encoded per photon and an enhanced security as apparent from the next section. Notably, as seen in section 2.7, $d+1$ mutually unbiased bases could be used for the QKD protocol. More mutually unbiased bases increase the security of the protocol [26], but come with lower key rates in case all bases are used with the same probability. Experimental tomography with high-dimensional states via mutually unbiased bases has been demonstrated [85].

\subsubsection{Eavesdropping}

The remaining question is if and under which circumstances the BB84 protocol is secure [26]. To answer this question, one has to consider the attack strategy of the eavesdropper. For the following subsections, the security of the $d$-dimensional two-bases BB84 protocol is discussed.

\section{Intercept resend attack}

The most straightforward attack strategy of Eve is called intercept resend. Eve intercepts a fraction $\eta$ of all photons sent via the quantum channel and performs the same projective measurements as Bob would do. Because Alice and Bob will only generate a key with photons Bob received, Eve needs to resend the same photon (still projected onto her measurement result). There are two scenarios. First, Alice and Eve randomly chose the same basis. Hence, the photon state is not changed by the measurement. Eve has the full information of Alice's sent information and no error is introduced by eavesdropping. But in the second 
scenario, Alice and Eve chose mutually unbiased bases. That means that the measurement outcome does not reveal any information about the sent information. The state is projected to one of the $d$ outcomes. Hence, even if Alice and Bob choose the same basis, the probability that Bob measures the correct symbol is only $1 / d$. Averaged over long keys, Eve will introduce an error rate of $Q=\eta 0.5 \frac{d-1}{d}$ by at best only obtaining half the information $I_{E}=\eta 0.5 H(A)$. For this attack, the secret fraction [86] is

$$
r=\max \left\{I(A ; B)-I_{E}, 0\right\} .
$$

Assuming that all errors are caused by the eavesdropping, the mutual information is given by equation (2.11)

$$
I(X ; Y)=\log _{2}(d)+(1-Q) \log _{2}(1-Q)+Q \log _{2}\left(\frac{Q}{d-1}\right)
$$

by substituting $F=1-Q$. No secret key can be extracted if $I(A ; B)<I_{E}$.

\section{Individual attacks}

The intercept resend attack is a special case of a more general attack called individual attack. As the name suggests, Eve attacks all signals from Alice to Bob individually and in the same way. The most general measurement she can perform is to let the signal from Alice interact with an ancilla, which she keeps while the signal goes to Bob. Eve doesn't have a quantum memory and performs her measurements on the ancillas before the postprocessing. As a result Alice, Eve and Bob share a classically correlated set of symbols. The information Eve gathers is

$$
I_{E}=\max _{\text {Eve }} I(A ; E),
$$

where the maximization is performed over Eve's attack strategies. The secret fraction is then given by the Csiszár-Körner bound [86] by replacing $I_{E}$ in equation (2.26) with the value from equation (2.28). A secret key can be generated for a fidelity fulfilling the inequality [44]

$$
F>\frac{1}{2}\left(1+\frac{1}{\sqrt{d}}\right) .
$$

\section{Collective attacks}

Collective attacks lift the restriction for Eve not to have a quantum memory. She can keep her ancillas as long as she wishes and can perform the best measurement given her knowledge, which is a collective measurement. The secret fraction in this case is given by the Devetak-Winter bound [87]

$$
\lim _{N \rightarrow \infty} r=S(X \mid E)-H(X \mid Y)
$$


where $S(X \mid E) \equiv S(X, E)-S(E)$ and $H(X \mid Y) \equiv H(X, Y)-H(Y)$ are the conditional von Neumann [88] and Shannon entropies, respectively. A secret key can be generated for a fidelity fulfilling the inequality [44]

$$
F \log _{2}\left(\frac{1}{F}\right)+(1-F) \log _{2}\left(\frac{d-1}{1-F}\right)<\frac{\log _{2} d}{2} .
$$

\section{Finite-key formalism}

So far, infinitely long keys are assumed. In case of finite key length, one needs to add corrections to equation (2.30), which had been studied in [89-91]. In this BB84-like coding, the two bases are used in an asymmetric way [92]. One basis is chosen with probability $p_{1}$ and is used to create the key between Alice and Bob. The second basis chosen with probability $p_{2}=1-p_{1}$ is used to detect eavesdropping and to estimate Eve's knowledge. From $N$ signals received by Bob, $2 N p_{1} p_{2}$ are removed in sifting, while $n=N p_{1}^{2}$ is the raw key length and $m=N p_{2}^{2}$ signals are used for estimating Eve's knowledge. The finite key length $N$ leads to several corrections of equation (2.30). The first correction is the factor $n / N=p_{1}^{2}$. For infinite key length, even a small part $m$ of the key is enough for error estimation and $p_{1} \approx 1$. To detect eavesdropping, the average error rate $Q$ needs to be estimated with finite samples. Assuming fluctuations of this rate with a standard deviation of $\Delta Q$, an upper bound of the estimated error rate is $Q+\frac{\Delta Q}{\sqrt{m}}$. Therefore, the conditional von Neumann entropy in equation (2.30) can be replaced by

$S(X \mid E) \leq \log _{2} d+\left(Q+\frac{\Delta Q}{\sqrt{m}}\right) \log _{2} \frac{Q+\frac{\Delta Q}{\sqrt{m}}}{d-1}+\left(1-Q-\frac{\Delta Q}{\sqrt{m}}\right) \log _{2}\left(1-Q-\frac{\Delta Q}{\sqrt{m}}\right)$.

Further, error correction and privacy amplification can fail with probabilities $\epsilon_{E C}$ and $\epsilon_{P A}$, respectively. The combination of these factors yield the lower bound for the secret key rate [89]

$$
\begin{aligned}
r_{N} & =\frac{n}{N}\left(\log _{2} d+\left(Q+\frac{\Delta Q}{\sqrt{m}}\right) \log _{2} \frac{Q+\frac{\Delta Q}{\sqrt{m}}}{d-1}+\left(1-Q-\frac{\Delta Q}{\sqrt{m}}\right) \log _{2}\left(1-Q-\frac{\Delta Q}{\sqrt{m}}\right)\right. \\
& \left.-H(A \mid B)-\frac{1}{n} \log _{2} \frac{2}{\epsilon_{E C}}-\frac{2}{n} \log _{2} \frac{1}{\epsilon_{E C}}-(2 d+3) \sqrt{\frac{\log _{2}\left(\frac{2}{\bar{\epsilon}}\right)}{n}}\right)
\end{aligned}
$$

where $\bar{\epsilon}$ is the theoretical failure probability of the mathematical estimates using smooth Renyi entropies [91, 93].

It should be mentioned that not all attack models are covered here. Every experimental realization of QKD can introduce quantum side channels due to device imperfections, which can be exploited by an eavesdropper without adding 
errors [39] and with hacking attacks [94]. Other attacks are based on the photon number statistics of the light source [29,30]. In case of multiphoton states, a beam splitter just next to Alice would allow Eve to read a part of the message and hide her interference in the losses by setting up a lossless quantum channel to Bob and to gain information without introducing errors. A solution to that is to vary the laser intensity throughout the protocol by using decoy states [95].

\subsection{Encoding}

In this thesis, the information is encoded in the transverse position of photons for QKD as first demonstrated by Walborn [55]. Let $f(\boldsymbol{r})$ be the detection amplitude on a $\mathrm{x}, \mathrm{y}$-plane, then

$$
|\psi\rangle=\int|\boldsymbol{r}\rangle\left\langle\mathbf{r}\left|d^{2} \boldsymbol{r}\right| \psi\right\rangle=\int f(\boldsymbol{r})|\boldsymbol{r}\rangle d^{2} \boldsymbol{r}
$$

is the single-photon state at that plane. Now, a position measurement is performed on state $|\psi\rangle$. The position is a continuous variable, which changes the sums in section (2.4) to integrals. Hence, the measurement operator fulfills the completeness relation

$$
\int\left|\boldsymbol{r}^{\prime}\right\rangle\left\langle\boldsymbol{r}^{\prime}\right| d^{2} \boldsymbol{r}^{\prime}=\mathbb{1},
$$

with $\left\langle\boldsymbol{r}^{\prime} \mid \boldsymbol{r}\right\rangle=\delta^{2}\left(\boldsymbol{r}^{\prime}-\boldsymbol{r}\right)$. Now equation (2.13) gives the probability to detect the photon at position $r^{\prime}$

$$
p\left(\boldsymbol{r}^{\prime}\right)=\left\langle\psi|| \boldsymbol{r}^{\prime}\right\rangle\left\langle\boldsymbol{r}^{\prime}|| \psi\right\rangle=\left|f\left(\boldsymbol{r}^{\prime}\right)\right|^{2} .
$$

The single-photon wave function can be expressed in k-space by using the completeness relation on equation (2.34)

$$
\begin{aligned}
|\psi\rangle & =\int f(\boldsymbol{r})|\boldsymbol{r}\rangle d^{2} \boldsymbol{r}=\iint f(\boldsymbol{r})\langle\boldsymbol{k} \mid \boldsymbol{r}\rangle|\boldsymbol{k}\rangle d^{2} \boldsymbol{k} d^{2} \boldsymbol{r} \\
& =\int \frac{1}{2 \pi} \int f(\boldsymbol{r}) e^{-i \boldsymbol{k} \cdot \boldsymbol{r}} d^{2} \boldsymbol{r}|\boldsymbol{k}\rangle d^{2} \boldsymbol{k},
\end{aligned}
$$

with $\langle\boldsymbol{k} \mid \boldsymbol{r}\rangle=\frac{1}{2 \pi} e^{-i \boldsymbol{k} \cdot \boldsymbol{r}}$ and the two-dimensional Fourier transform of the position amplitude

$$
\mathcal{F}(f(\boldsymbol{r}))[\boldsymbol{k}]=\frac{1}{2 \pi} \int f(\boldsymbol{r}) e^{-i \boldsymbol{k} \cdot \boldsymbol{r}} d^{2} \boldsymbol{r} .
$$

For a two-dimensional Gaussian function centered at $\boldsymbol{r}_{\mathbf{0}}=\left(x_{0}, y_{0}\right)$ with variance $\Delta x$ as the position amplitude

$$
f(\boldsymbol{r})=f(x, y)=\frac{1}{\sqrt{2 \pi \Delta r^{2}}} \exp \left(-\frac{\left(x-x_{0}\right)^{2}+\left(y-y_{0}\right)^{2}}{4 \Delta r^{2}}\right) .
$$


Together with equation (2.38) the amplitude in k-space is

$$
\mathcal{F}(f(x, y))\left[k_{x}, k_{y}\right]=\frac{2 \Delta r}{\sqrt{2 \pi}} \exp \left(-\Delta r^{2}\left(k_{x}^{2}+k_{y}^{2}\right)\right) \exp \left(-i\left(x_{0} k_{x}+y_{0} k_{y}\right)\right)
$$

The Fourier transform of a Gaussian is again a Gaussian function, where the variance in k-space can be defined as $\Delta k=1 /(2 \Delta r)$ to end up with the final result

$$
\mathcal{F}(f(x, y))\left[k_{x}, k_{y}\right]=\frac{1}{\sqrt{2 \pi \Delta k^{2}}} \exp \left(-\frac{k_{x}^{2}+k_{y}^{2}}{4 \Delta k^{2}}\right) \exp \left(-i\left(x_{0} k_{x}+y_{0} k_{y}\right)\right)
$$

The two variances fulfill the minimum uncertainty relation $[96,97]$

$$
\Delta r \Delta k=\frac{1}{2}
$$

Performing a measurement in k-space, equation (2.13) gives the outcome $\boldsymbol{k}^{\prime}$ with probability

$$
p\left(\boldsymbol{k}^{\prime}\right)=\left|\mathcal{F}(f(x, y))\left[k_{x}, k_{y}\right]\right|^{2}=\frac{1}{2 \pi \Delta k^{2}} \exp \left(-\frac{k_{x}^{2}+k_{y}^{2}}{2 \Delta k^{2}}\right) .
$$

Notably, this probability does not depend on the center of the Gaussian in $x-$ space $\boldsymbol{r}_{\mathbf{0}}=\left(x_{0}, y_{0}\right)$. A measurement in $\mathrm{k}$-space therefore reveals no information about the center position in $x$-space and vice versa. The $x$-space and $k$-space are Fourier transforms of each other and therefore form a set of mutually unbiased bases as described in section 2.7. Due to technical limitations, the basis vectors $|\boldsymbol{r}\rangle$ and $|\boldsymbol{k}\rangle$ can not be used for encoding. Due to finite apertures, $f(\boldsymbol{r})$ in equation (2.34) has a non-zero expansion in space. This leads to deviations from equation (2.23), which needs to be taken into account. The $x, y$-position of the photon can be measured by discrete detectors. 


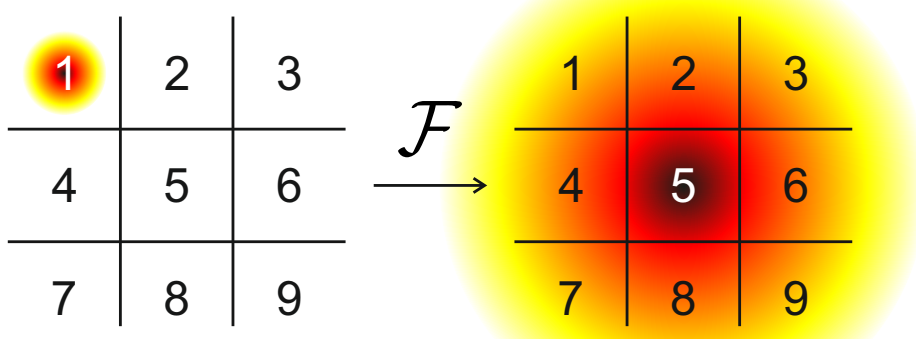

Figure 2.3 Illustration of 9 detectors and the probability function $p\left(\boldsymbol{r}^{\prime}\right)$. The photon wave function is centered on detector 1 , which results in a click of this detector (left). Fourier transforming erases the position information. The probability function spreads over all detectors (right).

The probability of the wave function to collapse at one particular detector (and causing a detector click) is the probability $p\left(\boldsymbol{r}^{\prime}\right)$ from equation (2.36) integrated over the area of the detector. In the case this is close to unity, centering another wavepacket on a second detector next to it has a negligible contribution to the area integral over the first detector. In that case, the two detectors can be labeled and a click on detector one is symbol one and a click on detector two is symbol two. Fourier transforming the single-photon wavepacket spreads the signal over the two detectors (with variance $\Delta k=1 /(2 \Delta r)$ in case of Gaussians), but the information concerning the center position is lost, as expected from a measurement in the mutually unbiased basis. The dimension of the two measurement bases is equal to the number of detectors. It can be extrapolated to $d$ detectors allowing a $d$-dimensional basis. This is illustrated in figure 2.3. Finally, it should be mentioned that also partial Fourier transforms [56] have been used as bases.

\subsection{Conclusion}

The concepts needed to understand the results in this thesis are introduced, starting with classical information theory. The quantum mechanical measurement operator and its consequences for quantum state discrimination are shown. The difference between quantum information and classical information is pointed out. Next, mutually unbiased bases are discussed as well as their implementation in the most common quantum cryptography protocol. Several attacker models for eavesdropping are presented. In the last section, the quantum states used for encoding are defined. 



\section{Upper bound on the mutual information}

\subsection{Introduction}

Up to this chapter, the information content of single photons was assumed to be only limited by the choice of the encoded quantum states as seen in section 2.6. If one considers free-space line-of-sight communication with light, one has to deal with and counteract several sources of noise [98, 99]. This includes pointing errors caused by misalignment of the transmitting optics and vibrations as well as earthquakes [100]. Another source is atmospheric turbulence caused by wind and temperature gradients [101]. Under realistic conditions, also the detection efficiency of detectors and their dark counts must be taken into account. The information capacity of spectrally [102] and temporally [43] encoded photons had been analysed. In this chapter, the upper limit of information which can be carried by spatially encoded photons is investigated. The investigation into the maximum information encoded in a single pulse starts by introducing the multiphoton effect in the first section. The second section focuses on the contribution of detector noise. Finally, the last section adds transverse beam broadening by noisy transmission channels.

\subsection{Upper limit information encoding}

In this section, an upper limit to the transmitted information content is calculated under noiseless conditions. The number of photons per signal pulse $N_{\mathrm{p}}$ can be larger than one. A number of $N_{d}$ (orthogonal) detectors is used to read out the signal. The detectors don't have photon number-counting capabilities. Simultaneous arrival of several photons results in only one detector click, just as for one photon. For this reason, we assume each detector has only a single photon incident and the number of distinguishable symbols $N_{s}$ is given by the binomial coefficient

$$
N_{s}=\left(\begin{array}{l}
N_{d} \\
N_{p}
\end{array}\right)=\frac{N_{d} !}{N_{p} !\left(N_{d}-N_{p}\right) !} .
$$

This number reaches its maximum when the number of symbols equals half the number of detectors. 

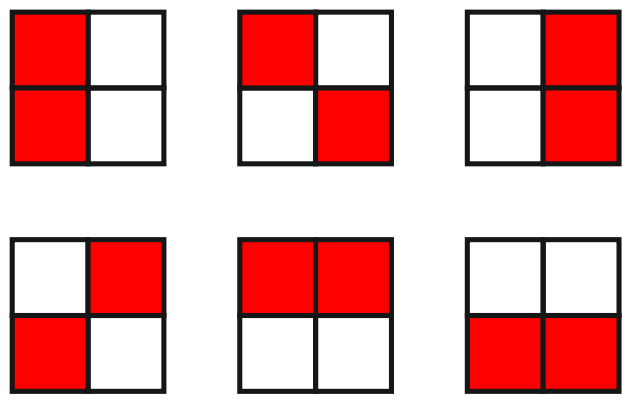

Figure 3.1 Illustration for $N_{d}=4$ and $N_{p}=2$ resulting in $N_{s}=6$ possible symbols. Two detector clicks (red) on four detectors yield six possible combinations.

The number of symbols is illustrated in figure 3.1. Next, the mutual information as described in section 2.3 is calculated. As required for the upper limit, all symbols are assumed to be sent with the same probability and are orthogonal to each other. In general, the symbols can have a non-zero overlap as apparent from figure 3.1. Here, the two upper left symbols share the click in the top left detector. Hence, they are not orthogonal. On the other hand, non-orthogonality will only reduce the accessible information as discussed in section 2.6. The joint probability to find symbol $x$ from the sender alphabet $X$ (with $N_{s}$ elements) and the symbol $y$ from the receiver alphabet $Y$ is

$$
P(x \in X, y \in Y)=\frac{\delta_{x y}}{N_{s}} .
$$

Since all states are assumed to be orthogonal, the number of symbols equals the dimension of the system. The probability to detect a symbol $y \in Y$ is

$$
P(y)=P(x)=\frac{1}{N_{s}}
$$

for an equal sent probability $P(x)$ of all symbols $x \in X$. Inserting these probabilities in equation (2.7) provides the mutual information as

$$
I(X ; Y)=\log _{2}\left(N_{S}\right) .
$$

The expression for the upper bound can be rewritten by using the Stirling approximation. The number of detectors is assumed to be larger than the number of photons $N_{d} \gg N_{p} \gg 1$. In combination with equation (3.1) and equation (3.4), the upper limit for the mutual information of one signal pulse is

$$
\begin{aligned}
I(X ; Y) & \approx \frac{1}{2} \log _{2}\left(\frac{N_{d}}{2 \pi N_{p}\left(N_{d}-N_{p}\right)}\right)+N_{d} \log _{2}\left(\frac{N_{d}}{N_{d}-N_{p}}\right) \\
& -N_{p} \log _{2}\left(\frac{N_{p}}{N_{d}-N_{p}}\right) .
\end{aligned}
$$



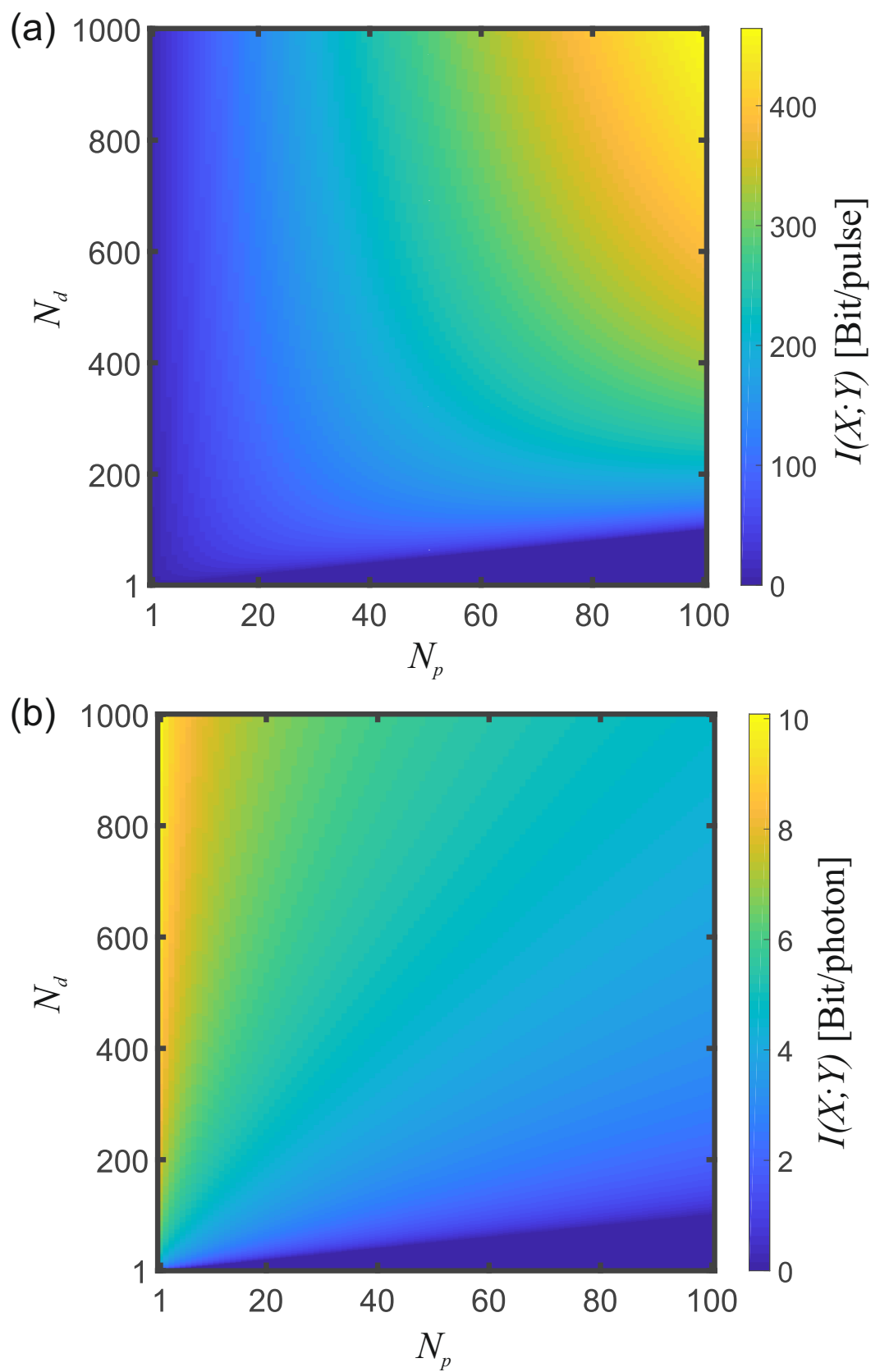

Figure 3.2 Surface plot of the upper bound on the mutual information $I(X ; Y)$ as a function of the number of detectors $N_{d}$ and the number of photons per signal $N_{p}$. Graph (a) shows the mutual information per pulse, while graph (b) is normalized to the number of photons per pulse. 
Figure 3.2 depicts the behavior of the mutual information as a function of the number of detectors and the number of photons per pulse. Equation 3.5 was used for plotting. The relative error in the plotted range stayed below $1.8 \%$. As seen in graph (a), its upper bound increases with both variables. The reason for that is the binomial coefficient in equation (3.1). Nevertheless, if the photon efficiency is taken into account, graph (b) points out that a pulse of a single photon has the highest possible mutual information per photon. Another problem is that despite great effort [103] multiphoton states are hard to create.

\subsubsection{Upper limit including detector noise}

Aspiring to a more realistic description, the influence of detector noise needs to be analyzed. As in the previous section, signal pulses with at maximum one photon per detection area are considered. The detection of these signals requires a special class of single-photon sensitive cameras. Their high sensitivity comes at the price of having finite dark count probability. The number of symbols is equal to equation (3.1) with $N_{p}$ the number of photons in a signal pulse and $N_{d}$ the number of detectors. If the number of detector clicks does not match the number of photons in the signal pulse, this measurement result will be ignored, as this is an indubitable sign that a noise event occurred. Per detector, four distinct events are possible. The two ideal scenarios are the correct negative and correct positive: No photon was sent and no photon is measured with probability $K_{00}$ or a photon is sent and causes a click on the detector with probability $K_{11}$. The two unwanted events are the false positive and false negative: No photon was sent, but the detector clicks with probability $K_{01}$ or a photon is sent, but is not detected with probability $K_{10}$. With two of these probabilities, one can calculate the probability to correctly measure the symbol as

$$
R=K_{00}^{N_{d}-N_{p}} K_{11}^{N_{p}} .
$$

In this case, all $N_{p}$ detectors with incident photons clicked, while in $N_{d}-N_{p}$ times no dark counts occurred. The probability to detect a wrong symbol is not $1-R$, since measurements where the number of detection events is not equal to the number of incident photons $N_{p}$ are discarded, and a correct count is not synonymous with correct measurements at each detector. For a constant and correct number of detection events, every photon which is not causing a detector click needs to be compensated by a dark count of a falsely counting detector. The probability for $k$ false detector clicks is therefore given by

$$
W_{k}=K_{00}^{N_{d}-N_{p}-k} K_{11}^{N_{p}-k} K_{01}^{k} K_{10}^{k} .
$$

Here, $N_{p}-k$ detectors with incident photons clicked, while in $N_{d}-N_{p}-k$ times, no dark counts occurred. To fulfill the frame condition of $N_{p}$ total clicks, $k$ detectors which have not clicked click with probability $K_{01}$, while $k$ detectors 
don't click with probability $K_{10}$. Without dark counts above the required number for $N_{p}$ clicks, $k=0$ and $W_{0}=R$. To calculate the total error probability $W$, the probability $W_{k}$ need to be summed multiplied with the number of permutations. This leads to

$$
W=\sum_{k=1}^{\min \left(N_{p}, N_{d}-N_{p}\right)} W_{k}\left(\begin{array}{c}
N_{d}-N_{p} \\
k
\end{array}\right)\left(\begin{array}{c}
N_{p} \\
k
\end{array}\right) .
$$

The average probability to measure the wrong symbol is $\frac{W}{N_{s}-1}$. The joint probability is

$$
\begin{array}{ll}
P(x, y)=\frac{R}{N_{s}(R+W)} & \forall x=y \\
P(x, y)=\frac{W}{N_{s}\left(N_{s}-1\right)(R+W)} & \forall x \neq y .
\end{array}
$$

The probability for a symbol $x$ being sent is $P(x)=\frac{1}{N_{s}}$ and is equal to the probability that a symbol $y$ is received $P(y)=\frac{1}{N_{s}}$. In contrast to section 3.2, the joint probability distribution does have off-diagonal elements, which arise from the detection noise. The probabilities can be used to calculate the mutual information using equation (2.7). The sum in this equation can be split in one term with the diagonal elements of $P(x, y)$ and one with the off-diagonal elements of $P(x, y)$. The final expression is

$$
I(X ; Y)=\frac{R}{R+W} \log _{2}\left(\frac{N_{s} R}{R+W}\right)+\frac{W}{R+W} \log _{2}\left(\frac{\frac{N_{s}}{N_{s}-1} W}{R+W}\right)
$$

or as a function of the ratio $a=\frac{W}{R}$

$$
\begin{aligned}
I(X ; Y) & =\frac{1}{1+a} \log _{2}\left(\frac{N_{S}}{1+a}\right)+\frac{a}{1+a} \log _{2}\left(\frac{\frac{N_{S}}{N_{S}-1} a}{1+a}\right) \\
& =\log _{2}\left(N_{S}\right)+\log _{2}\left(\frac{1}{1+a}\right)+\frac{a}{1+a} \log _{2}\left(\frac{a}{N_{S}-1}\right) .
\end{aligned}
$$

The mutual information is shown in figure (3.3). As expected, the mutual information increases for smaller ratios $a=\frac{W}{R}$. The larger the probability to detect the right symbol $R$ and the lower the probability to measure a wrong symbol $W$, the larger the mutual information. Moreover, the mutual information increases with the number of symbols, as already seen in the previous section. 


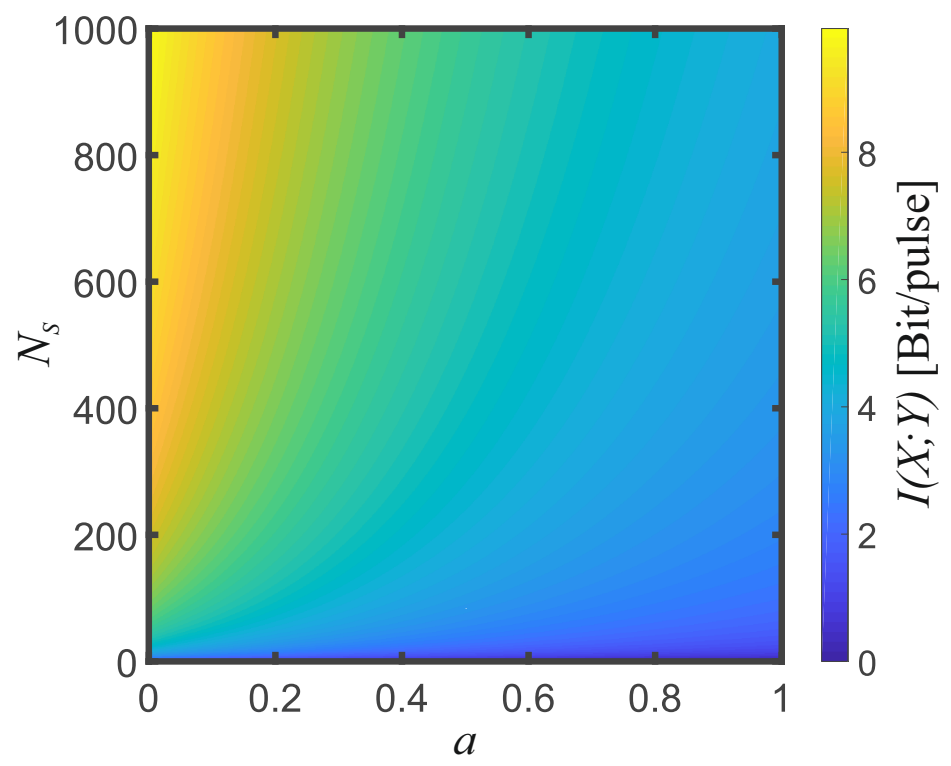

Figure 3.3 Surface plot of the upper bound on the mutual information $I(X ; Y)$ as a function of the number of symbols $N_{S}$ and the ratio $a=\frac{W}{R}$.

In this section, the number of detection events was considered equal to the number of signal photons. As a consequence of this restriction, the loss of the signal corresponds to events where these numbers are unequal. That leads to a total loss of $L=1-W-R$, which takes all events into account not being detected as wrong or right symbol into account. This loss scales polynomial in the number of detectors and signal photons. For a general discussion, this restriction needs to be lifted. In this model, a $N_{p}$ photon Fock state is detected by $N_{d}$ detectors with an efficiency of $\eta$ each. If the average number of dark counts is $N_{\text {dark, }}$ each detector's probability for a dark count is $\frac{N_{\text {dark }}}{N_{d}}$. The fidelity to detect the correct symbol is

$$
F=\frac{N_{p}\left(\eta+\frac{N_{\text {dark }}}{N_{d}}\right)}{N_{p}\left(\eta+\frac{N_{\text {dark }}}{N_{d}}\right)+\left(N_{d}-N_{p}\right) \frac{N_{\text {dark }}}{N_{d}}}=\frac{N_{p}\left(\eta+\frac{N_{\text {dark }}}{N_{d}}\right)}{N_{p} \eta+N_{\text {dark }}} .
$$

This fidelity can be inserted in equation (2.11) to find the mutual information

$$
I(X ; Y)=\log _{2}\left(N_{S}\right)+F \log _{2}(F)+(1-F) \log _{2}\left(\frac{1-F}{N_{S}-1}\right)
$$




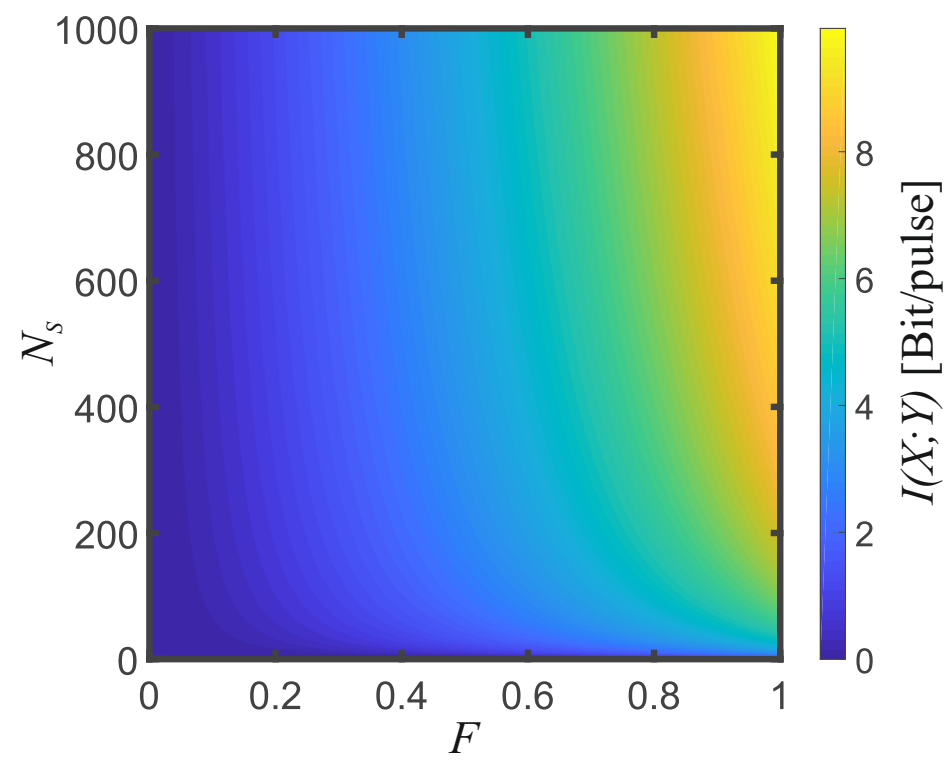

Figure 3.4 Surface plot of the upper bound on the mutual information $I(X ; Y)$ as a function of the number of symbols $N_{S}$ and the fidelity $F$.

The influence of the fidelity on the mutual information is shown in figure 3.4. The information per pulse increases with the fidelity and reaches its maximum at a fidelity of one which corresponds to the noise-free case with a mutual information of $\log _{2}\left(N_{S}\right)$.

\subsubsection{Upper limit including channel noise}

As discussed in the previous section, if one wants to increase the mutual information in a photon-efficient way, one should consider a single photon on a large number of detectors. This is why in this section the number of photons is limited to one photon per signal pulse. The detectors are arranged in a twodimensional detection array and the photon is steered to one specific detector. The channel noise is introduced as a broadening function $F\left(x-x_{0}, y-y_{0}, \sigma\right)$ of the focus with a width of $\sigma$ around a central point $\left(x_{0}, y_{0}\right)$ in the detector array. The overall probability of dark counts is assumed to be small compared to the detector efficiency, which allows to simplify the analysis by only taking singlephoton detection into account. Due to the broadening of the focus of the photon and the two-dimensional arrangement of the detectors, cross-talk between the detectors becomes significant. The size of the detectors could be increased to minimize the cross-talk, but in realistic scenarios with finite-size apertures, this is not possible. The other extreme would be a focus spread over many detectors, which minimizes the mutual information. To calculate the mutual information 
from equation 2.7, the joint probability distribution $P(X ; Y)$ needs to be known. A single-photon Fock states with symmetric foci with width $\sigma$ is incident on detectors with an efficiency of $\eta$. The average number of dark counts per symbol is $N_{\text {dark. }}$. The joint probability distribution $P(X, Y)$ has two contributions. First, the actual signal which is simulated by the broadening function $F\left(x-x_{0}, y-y_{0}, \sigma\right)$ weighted with the detector efficiency $\eta$. Second, the contribution of the detector dark counts. They are assumed to form a constant noise floor of $\frac{N_{\text {dark }}}{N_{d}}$ per detector. These two contributions need to be summed up for each symbol, forming a statistical function $M(X, Y)$ for every symbol from the sent alphabet $x \in X$ and receiver alphabet $y \in Y$. For convenience, the detectors are numbered from 1 to $N_{d}$ from left to right and top to bottom and quadratic detector arrays are assumed. For each symbol $x$, this statistical function can be calculated by integrating the intensity of the focus over the detectors yielding

$$
\begin{aligned}
M(x, y) & =\eta \int_{\text {floor }\left(\frac{y}{\sqrt{N_{d}}}\right)}^{\text {floor }\left(\frac{y}{\sqrt{N_{d}}}\right)+1} \int_{\left(y \bmod \sqrt{N_{d}}\right)-1}^{\left(y \bmod \sqrt{N_{d}}\right)} \\
& F\left(k-\text { floor }\left(\frac{x}{\sqrt{N_{d}}}\right)-0.5, l-\left(x \bmod \sqrt{N_{d}}\right)+0.5, \sigma\right) \quad d k d l \\
& +\frac{N_{\text {dark }}}{N_{d}} .
\end{aligned}
$$

The floor $\left(x / \sqrt{N_{d}}\right)+1$ translates the symbol number $x$ to a row number of a two-dimensional quadratic array, while the $x \bmod \sqrt{N_{d}}$ is the row number. In case of a Gaussian broadening

$$
F\left(x-x_{0}, y-y_{0}, \sigma\right)=\frac{1}{2 \pi \sigma^{2}} \exp \left(-\frac{\left(x-x_{0}\right)^{2}+\left(y-y_{0}\right)^{2}}{2 \sigma^{2}}\right) .
$$

Finally, the normalization criterion of the joint probability

$$
\sum_{x \in X, y \in Y} P(x, y)=1
$$

needs to be fulfilled, resulting in

$$
P(x, y)=\frac{M(x, y)}{\sum_{x \in X, y \in Y} M(x, y)} \approx \frac{M(x, y)}{N_{d}\left(\eta+N_{\text {dark }}\right)} .
$$

For the maximum mutual information the sent and received probabilities $P(X)=$ $P(Y)=\frac{1}{N_{S}}$ are equal for all symbols. With these values, the mutual information can be computed by equation (2.7). 


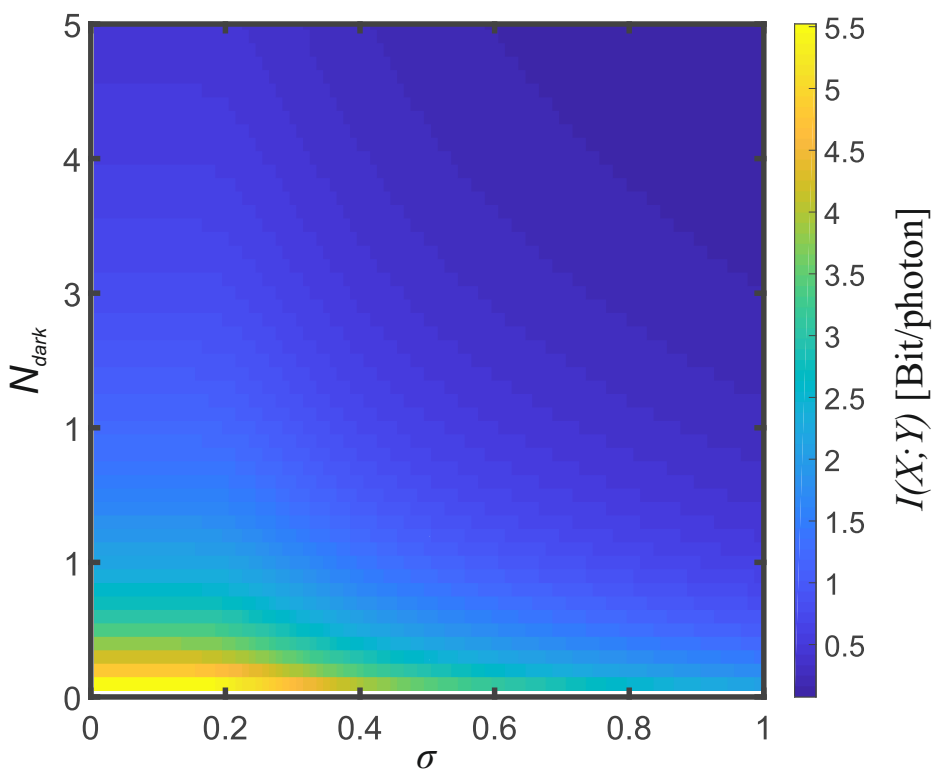

Figure 3.5 Surface plot of the upper bound of the mutual information as a function of the number of dark counts $N_{\text {dark }}$ and the width of the Gaussian focus $\sigma$. The number of detectors is kept constant at $N_{d}=100$ with an efficiency of $\eta=0.9$.

Figure 3.5 illustrates the mutual information per photon as a function of the number of dark counts and the width of the focus. A large number of dark counts reduces the mutual information. Similarly, a broader focus reduces the mutual information. In the limit $\sigma \rightarrow 0$, this result is equal to the one in the previous section. For finite $\sigma$, considering the same parameters as in equation (3.15), the mutual information obtained by equation (3.19) is larger. Even though the probability to trigger the target detector is identical, the fact that neighboring detectors have a higher hit probability leads to a larger mutual information.

\subsection{Conclusion}

This chapter derives the upper bound on the mutual information between a sender and a receiver for a specific model. The first section gives an expression for the maximum information which can be encoded. It is shown that in order to maximize the information per photon, a single photon should be encoded per signal pulse. The following section introduces detector noise into the problem. Detector noise lowers the mutual information per photon. Finally, the last section discusses the influence of beam broadening to the mutual information. 



\section{Transmitting more than $\mathbf{1 0}$ bit with a single photon}

Encoding information in the position of single photons has no known limits, given infinite resources. Using a heralded single-photon source and a Spatial Light Modulator (SLM), we steer single photons to specific positions in a virtual grid on a large-area spatially resolving photon-counting detector (ICCD). We experimentally demonstrate selective addressing any location (symbol) in a 9072 size grid (alphabet) to achieve 10.5 bit of mutual information per detected photon between the sender and receiver. Our results can be useful for very-highdimensional quantum information processing.

\subsection{Introduction}

Its weak interaction with the environment makes light ideal for sharing information between distant parties. For this reason, light is used to transmit information all around the world. With the advent of single-photon sources, a new class of applications has emerged. Due to their quantum properties, single photons are used to entangle quantum systems or to do quantum cryptography [27]. One famous example is Quantum Key Distribution (QKD) using the BB84 protocol [13] to securely build up a secret shared key between Alice and Bob. The security of this method is based on the no-cloning theorem [16], which forbids copying quantum states. The standard implementation of the BB84 protocol uses the twodimensional polarization basis to encode information in photons. Therefore the alphabet contains only two symbols " 0 " and " 1 ", limiting the information content per photon to $1 \mathrm{bit}$. Increasing the dimension of the basis using a large alphabet increases the information content per photon together with an improvement in the security $[26,44,46]$. This is the motivation to employ larger alphabets using orbital angular momentum [48-50], time binning [25, 52, 53] or spatial translation [55-57]. Among the spatial encoding schemes, Orbital Angular Momentum (OAM) states have been proposed for high-dimensional information encoding [104]. However in a practical scenario, assuming a sender-receiver configuration with apertures of finite size, a diffraction-limited spot translated in space or Laguerre-Gauss modes has a higher capacity limit than the subset of pure OAM states $[59,60]$. This makes spatial positioning of light or equivalently, tilting of plane waves, an ideal method for increasing the information content per photon. Interestingly, given infinite resources, there is no known upper bound for the information content transmitted by single photons. For example, using one mole $\left(6.022 \cdot 10^{23}\right)$ of ideal position-sensitive single-photon detectors leads to an

The content of this chapter has been published as: T. B. H. Tentrup, T. Hummel, T. A. W. Wolterink, R. Uppu, A. P. Mosk, and P. W. H. Pinkse, "Transmitting more than 10 bit with a single photon," Opt. Express 25, 2826-2833 (2017). 
information content of 79 bit per detected photon. Clearly, this is out of reach in a practical situation. A very relevant question therefore is what can be realised experimentally.

In this chapter we report our experiment in which we have deterministically encoded more than 10 bit of information into a single photon. We have employed $2^{3}$ times more symbols than previous work in spatial encoding, which reported 7 bit per photon as highest value for random keys [57], and is comparable to what has been achieved in temporal and polarization encoding[105].

\subsection{Methods}

\subsubsection{Setup}

The setup is illustrated in Fig. 4.1. We use a Spontaneous Parametric DownConversion source [112] to produce photon pairs. A $790 \mathrm{~nm}$ mode-locked picosecond laser with a pulse repetition rate of $76 \mathrm{MHz}$ is frequency doubled to $395 \mathrm{~nm}$ in a LBO crystal. The frequency-doubled light is focussed in a Periodically Poled Potassium Titanyl Phosphate (PPKTP) crystal, which spontaneously produces orthogonally polarized photon pairs at a wavelength of $790 \mathrm{~nm}$. The entangled photon pair is separated at a Polarizing Beam Splitter into two singlephoton Fock states. One of these photons is sent to a Single Photon Counting Module acting as a herald, while the second photon is directed through a $28.5 \mathrm{~m}$ single-mode fiber with a throughput of $47 \%$ to the encoding setup. For freespace communication, one needs an encoding device at the sender position and a decoding device on the receiver position. As encoding device we use a phaseonly Spatial Light Modulator to modulate the wavefront of the single photons. By writing a blazed grating on the SLM, we change the reflection angle within an angular range of $0.8^{\circ}$ in vertical and horizontal direction with a diffraction efficiency of $76 \%$ in the first order. The Fourier transform of the SLM is imaged with a $1 \mathrm{~m}$ focal length lens in $2 \mathrm{f}$ configuration onto a large-area spatially resolving photon-counting detector. A bandpass filter at $800 \pm 40 \mathrm{~nm}$ is placed in front of the detector to block stray light.

\subsubsection{Detector}

The decoding device should be able to measure the arrival of a single photon in a single shot on a large area. One technology that can achieve this is the Intensified Charge-Coupled Device [113-120]. ICCDs provide nanosecond gating option, which reduces the amount of dark counts significantly and makes such an ICCD capable of heralded measurements, reducing dark counts to one per thousands of readouts. The dark counts of the ICCD have their origin in thermal electrons released by the photocathode of the ICCD. Additionally, residual gas atoms can be ionized by the electron avalanche within the Microchannel Plate (MCP) of 


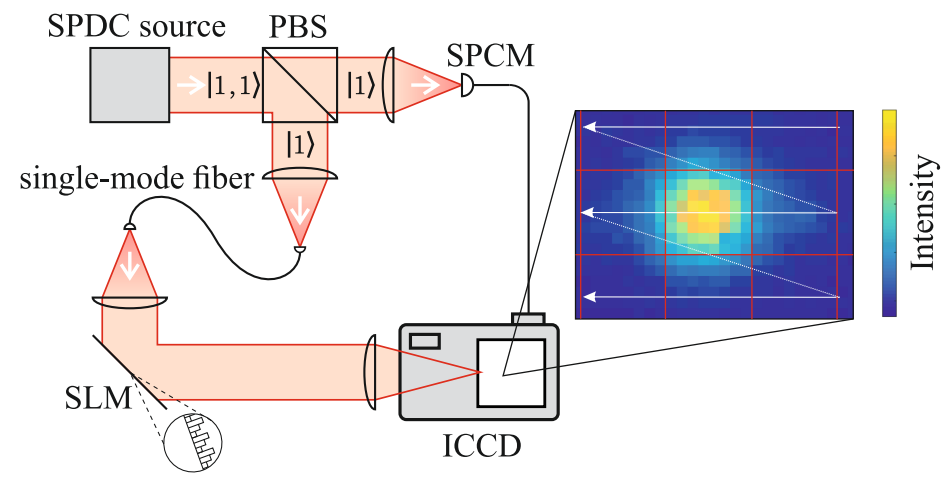

Figure 4.1 Schematic representation of the setup. The Type-II Spontaneous Parametric Down-Conversion (SPDC) source produces photon pairs, which are split by a Polarising Beam Splitter (PBS). One of the photons is detected by a Single-Photon Counting Module (SPCM) acting as a herald for an Intensified CCD (ICCD). The other photon is fiber-coupled and is incident on a Spatial Light Modulator (SLM). Its Fourier image is projected on the ICCD. The position of the focus is scanned by adjusting the blazed grating on the SLM, indicated by the arrows. An accumulated focus is shown in a zoom-in of the ICCD image integrated over an average of 1000 photons. The red lines show the $8 \times 8$ pixel binning of the symbols.

the intensifier. These ions are accelerated towards the photocathode by the MCP bias voltage, releasing secondary electron avalanches. An ion impact causes many more electrons than an incoming photon does. This leads to a local signal increase on the ICCD camera which is brighter than the signal produced by a single photon. Therefore these spurious ion signals can be filtered out in postprocessing. Our model (Andor iStar A-DH334T-18u-A3) has a photocathode quantum efficiency of $5 \%$. Each heralded photon from the SPDC source opens the ICCD gate for $2 \mathrm{~nm}$. For the measurements in Fig. 4.26 images with an exposure time of $0.1 \mathrm{~s}$ have been taken at each symbol. Single photon events were analyzed using threshold photon counting [120], with the threshold level set above the readout noise of the CCD camera. Attenuating the SPDC to a herald rate of $\sim 400 \mathrm{kHz}$, we measured on average 7.3 photon detections per symbol. The FWHM of the focus, with a constant phase pattern on the SLM and integrated over an average of 1000 photon detection events, was found to be $7.9 \pm 0.3$ pixels horizontally and $7.4 \pm 0.2$ pixels vertically.

\subsubsection{Encoding}

The target position of the photon on the ICCD is determined by the horizontal and vertical diffraction angle of the grating on the SLM. Although a scan mirror 
could be used for spatial encoding, a SLM is a more flexible tool. Via holography, the phase and amplitude of a wavefront can be manipulated, allowing to use complex wavefronts. Moreover, the path of the light can be corrected for disturbances using wavefront-shaping methods [121]. The SLM (pixel pitch: $20 \mu \mathrm{m}$, resolution: $800 \times 600$ pixels) is programmed with horizontal and vertical gratings to scan over the detection plane of the ICCD. The angular range of encoded alphabets is small $\left(\sim 0.33^{\circ}\right)$ and the grating pitch was over 5 pixels, which ensured high and uniform diffraction efficiency for all symbols. In order to define the sent symbol, the position on the ICCD has to be mapped to separate symbols. For this reason, a grid is defined on the ICCD. The pixels of the ICCD are binned together in detection areas of $8 \times 8$ or $12 \times 12$ pixels, forming an alphabet of 9072 or 4050 symbols, respectively. This rectangular map on the detection plane of the ICCD connects every detection area to an individual label, numbered from left to right and top to bottom.

\subsection{Result}

The spatial encoding of information is realized on a rectangular, virtual grid formed by binning the pixels of a camera. In case the entire camera chip would be used, the maximum information is $\log _{2}(1024 \cdot 1024)=20$ bit. If we fill in our experimental parameters, namely a signal-to-dark-count ratio of 10.08 and a focus size of 8 pixels in each direction on the ICCD, this number is reduced to 14.45 bit. In our experiment we used a grid of $112 \times 81$ areas of binned pixels, which are the 9072 symbols of our alphabet. This value corresponds to a maximum information content of $\log _{2}(9072)=13.15$ bit.

The light is directed to distinct symbols on the grid by scanning the focus using a SLM as a blazed grating. We assume that the sender uses an alphabet $X$ and the receiver an alphabet $Y$. To characterize the system, we sample the joint probability distribution $P(X, Y)$, which indicates the probabilities $p(x, y)$ to detect a certain symbol $y$ out of the alphabet $Y$ if a symbol $x$ out of the alphabet $X$ was sent. The result is plotted in Fig. 4.2. The readout noise of the ICCD was reduced by applying a threshold on the measured signal to only show the intensified signal. A diagonal line in the plot of the sent symbol versus the received would indicate maximal correlation. Indeed there is a strong correlation between the sent and received symbol set visible in graph (a), which shows the whole alphabet in a log-log plot. Graph (b) depicts a zoom into the first 200 of the 9072 symbols. Due to crosstalk between the symbols, off-diagonal lines are visible, which correspond to photons hitting the symbol above or below the target. The distance of 112 symbols between these lines and the diagonal corresponds to the column length of the grid written on the camera. The signal to the left and right of the diagonal is caused by crosstalk to the left and right symbol on the grid. Noise can also arise from dark counts of the ICCD. 


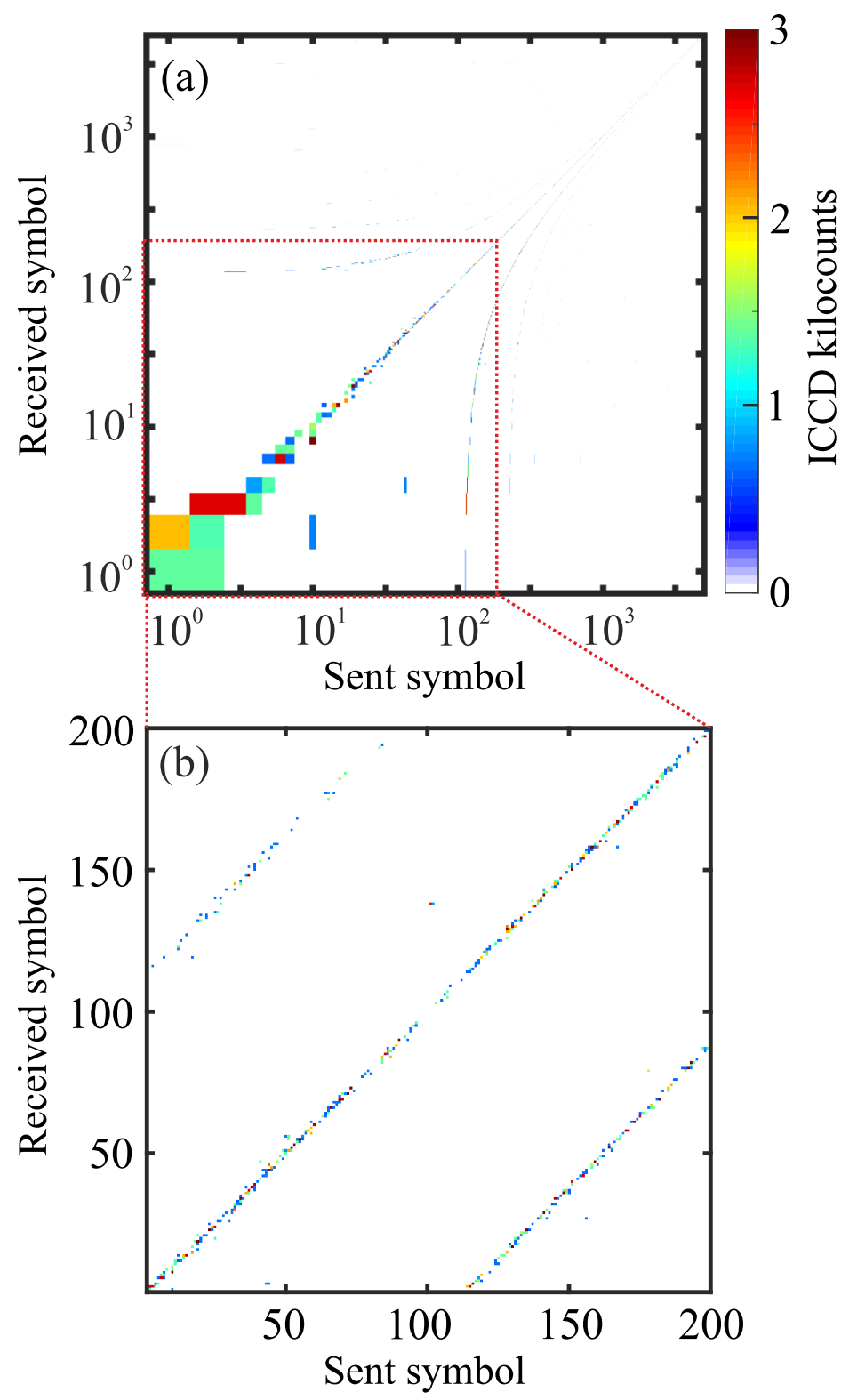

Figure 4.2 Measured ICCD counts in each of the measured symbols as a function of the sent symbol with a binning size of $8 \times 8$ pixels. The exposure time was $0.6 \mathrm{~s}$ for each symbol. Graph (a) illustrates the correlation between all 9072 symbols in a log-log plot. The graph (b) shows the measured correlation between the first 200 symbols in a linear plot. The measurement samples the joint probability distribution $P(X, Y)$. 
To quantify the information content per photon, we calculate the mutual information between sender and receiver. The mutual information $I(X: Y)$ is the measure of the average reduction in uncertainty about a sent symbol set $X$ that results from learning the value of the received symbol set $Y$; or vice versa, the average amount of information that $X$ conveys about $Y$ [65]. The mutual information per detection event of the sender-receiver system is mathematically represented as

$$
I(X: Y)=\sum_{x \in X, y \in Y} p(x, y) \log _{2}\left(\frac{p(x, y)}{p(x) p(y)}\right),
$$

where $p(x, y)$ is the probability that symbol $y$ is received when symbol $x$ is sent. $p(y)$ is the probability to measure symbol $y$ and $p(x)$ the probability that the sender encodes the symbol $x$. Theoretically, the mutual information depends on the number of symbols $N$, which has a maximum of $I_{\max }=\log _{2}(N)$, assuming $p(x)=1 / N$ for every symbol $x \in X$. In the experiment, we ensured the uniform probability of $x$ to realize the theoretical maximum in the absence of noise. The maximum number of symbols is limited by the finite size of the CCD. Using the presented binning size of the detection areas of $8 \times 8$, our theoretical limit is 13.15 bit.

In reality, the mutual information is not only limited by the number of symbols and the entropy of the sent alphabet, but also reduced by the crosstalk between the symbols arising from diffraction-limited focal spots as well as the noise from environment and detector. In order to reduce the crosstalk between the symbols, the binning size of the detection areas can be increased. This, however, reduces the number of symbols given the limited number of pixels on the detector. The blue circles in Fig. 4.3 show the dependence of the maximum mutual information for symbols made with different pixel bin sizes. The measured mutual information for $8 \times 8$ and $12 \times 12$ pixel bin sizes are represented as red circles. The measured data is lower than the theoretical limit. This can be understood from the average hit probability, indicated by the green + markers. Moreover, accounting for a finite signal-to-dark-photocount ratio between 10 and 100, leads to the expected mutual information, depicted as gray bars in the figure. As evident from the figure, there is a maximal mutual information given by physical limitations of crosstalk and noise. For large bin sizes with near-zero crosstalk between symbols, one can achieve very high mutual information of over 9 bit per photon. Given the FWHM spot size of approximately 8 pixels, we choose an $8 \times 8$ binning achieving a value of 10.5 bit of mutual information per detected photon.

To calculate the mutual information per sent photon, one has to take the losses in our setup into account. This includes the coupling losses into the single mode fibres of $55 \%$, the diffraction losses at the SLM of $24 \%$, the losses at the spectral filter of $30 \%$ and the losses at the detector due to the limited quantum efficiency of $5 \%$. This leads to a channel capacity of 0.1 bit per photon. 


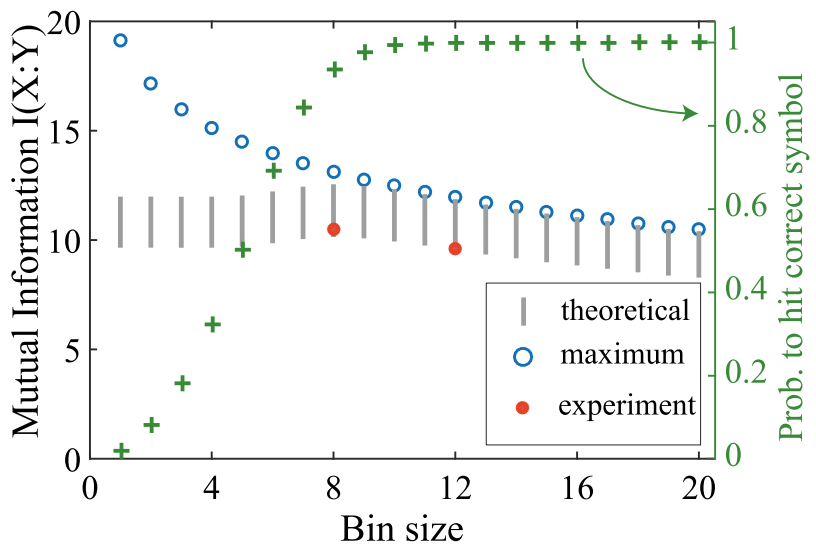

Figure 4.3 Dependence of the mutual information and the average hit probability in the correct symbol on the binning size of the detection areas. The blue circles represent the theoretical limit $I_{\max }$ given no noise or crosstalk. The red dots correspond to the measured mutual information for $8 \times 8$ and $12 \times 12$ pixel bin size. The theoretical mutual information is shown as gray bars in the figure, corrected for a signal-to-dark-count photon ratio between 10 and 100. The green + markers illustrate the average hit probability in the correct area for a finite focal diameter with FWHM of 8 pixels as shown in Fig. 4.1.

\subsection{Discussion}

For useful communication, the errors within the received message have to be corrected. An efficient way of error correction is the Low-Density Parity-Check Code (LDPC) [106]. In order to apply this error correction, all symbols of the set have to be translated into a bit string. Therefore we encode the $x$ and $y$ position of our symbols independently, each allocating half the bits. Since the dominant noise term is the crosstalk between the neighbouring symbols, we use a Gray code [107] for each direction. This causes only one bit flip for an erroneous detection by a neighbouring symbol either in $x$ or the $y$ direction.

Figure 4.4 shows the Bit Error Rate (BER) after error correction with LDPC versus the BER of the received bit string. The LDPC code was set to the halfrate LDPC used in digital television broadcast standard DVB-S.2. The coloured vertical bars indicate the estimated BER in case of $8 \times 8$ and $12 \times 12$ binning. The estimation takes the measured crosstalk between the symbols into account. Their left and right edges indicate a signal-to-dark-count photon ratio of 100 and 10, respectively. For the ICCD used in these measurements, this ratio is 10.07. Other commercially available ICCD have ratios close to 100, explaining the choice of the other bound. Clearly, already a standard error-correction code allows a practically error-free communication with the present system. 


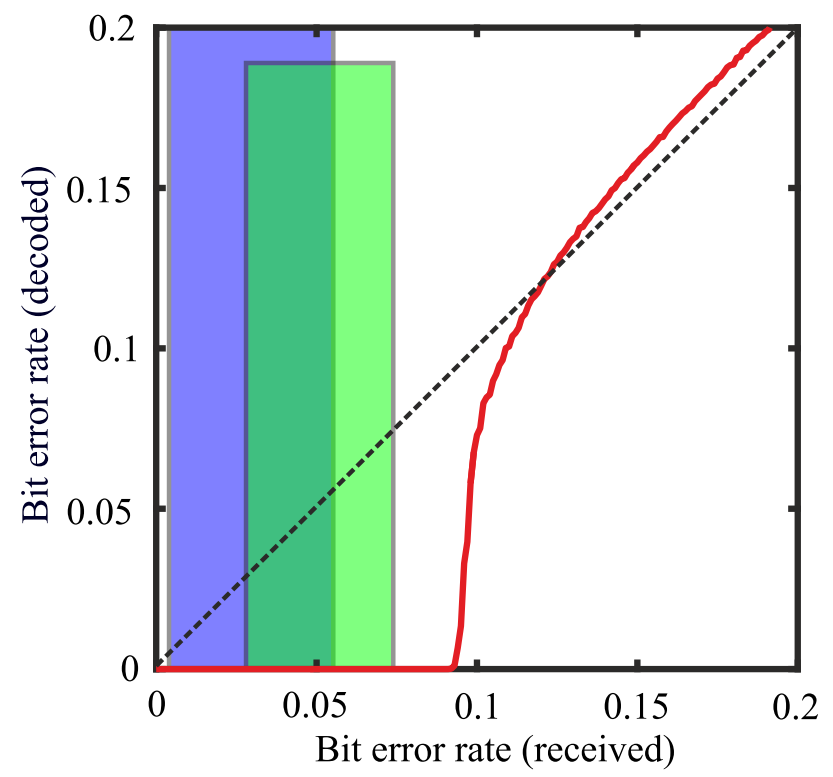

Figure 4.4 The Bit Error Rate (BER) of the received bit string versus the BER of the bit string after performing error correction. The dashed diagonal line represents the result without error correction. The vertical bars indicate the estimated BER of our experiment in case of $8 \times 8$ (green) and $12 \times 12$ (blue) binning. Their left and right edges indicate a signal-to-dark-count photon ratio of 100 and 10, respectively. 


\subsection{Conclusion}

In conclusion, we demonstrate high-dimensional encoding of single photons reaching 10.5 bit per photon. The capacity of this spatial encoding is only limited by the optics and the number of pixels on the detector. The channel capacity of 0.1 bit can be increased by reducing the losses in the system. The main contribution to losses in our setup arises from the low quantum efficiency $(\sim 5 \%)$ of the ICCD, which can be improved to $\sim 30 \%$ with different photocathode materials. This makes it possible to reach a signal-to-dark-count ratio of 100 and would bring the measured values closer to its theoretical maximum. Our results are directly applicable to free-space line-of-sight communication. If the scrambling of wavefronts in multimode fibers can be controlled [108, 109], a second and potentially more robust carrier for this high-dimensional encoding can be realised. A very promising direction for this work would then be the implementation of a large-spatial-alphabet encoding for quantum key distribution or high-dimensional quantum data locking [110, 111]. 



\section{$5 \quad$ Large-Alphabet QKD}

Most Quantum Key Distribution protocols use a two-dimensional basis such as HV polarization as first proposed by Bennett and Brassard in 1984. These protocols are consequently limited to a key generation density of 1 bit per photon. We increase this key density by encoding information in the transverse spatial displacement of the used photons. Employing this higher-dimensional Hilbert space together with modern single-photon-detecting cameras, we demonstrate experimentally large-alphabet Quantum Key Distribution with 1024 symbols and a shared information between sender and receiver of 7 bit per photon.

\subsection{Introduction}

We rely increasingly on the availability of affordable and high speed communication, which fosters the need of high key-rate generating cryptography. Recent progress in the development of quantum computers [122-126] threatens the widely used cryptographic methods, which rely on computational assumptions $[10,127]$. A possible solution is Quantum Key Distribution (QKD) of which the security is only based on quantum physics and not on any computational assumption. The first QKD protocol BB84 [13] uses the two-dimensional polarization basis to encode information in photons. Therefore, the alphabet is limited to two symbols, "0" and "1", with a maximum information content of 1 bit per photon. Since the generated key is used as a one-time pad, this is a bottleneck especially for encrypted video communication [37].

There are two approaches to increase the key generation rate. One is to increase the repetition rates of photon generation [41] and detection [42], which is inherently limited by dead times and jitter of the detectors [43]. The other approach is to exploit properties of photons besides the polarization to increase the dimensionality of the Hilbert space [45, 46]. A higher dimensional Hilbert space leads to a higher information content of the photons and finally increases the key generation rate. Moreover, the error rates introduced by eavesdropping are larger, resulting in an increased security [44].

Several methods of high-dimensional QKD have been demonstrated, including time-bin encoding [53,54, 128, 129], orbital angular-momentum states [48, 49, 51, 130] and transversal momentum states [55, 58]. Comparing the last two spatial encoding schemes, transversal momentum states have the following advantages. Assuming a realistic sender-receiver configuration with finite-size aper-

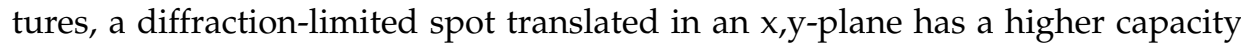
limit than the pure OAM states, since they form a subset of Laguerre-Gauss modes [131, 132]. Together with the ease of generating a Fourier-transformed

This chapter is based on: T. B. H. Tentrup, W. M. Luiten, R. van der Meer, P. Hooijschuur, and P. W. H. Pinkse, "Large-alphabet Quantum Key Distribution using spatially encoded light", in preparation. 
mutually unbiased basis with lens optics, spatial translation states of single photons are promising candidates for very-high-dimensional QKD.

In principle, a scan mirror could be used to change the spatial translation states of single photons. If one wants to correct for disturbances using wavefrontshaping methods [133], a Spatial Light Modulator (SLM) is needed. The SLM allows to change the phase and amplitude of a wavefront by use of holographic methods.

In this chapter, we experimentally demonstrate very-high-dimensional QKD with 1024 distinguishable symbols in two mutually unbiased bases with a shared information of 7 bit per sifted photon. This value is higher than previously reported values of 2.05 bit for OAM states [48] and comparable to the values demonstrated in time-energy QKD [53]. We give finite-key security arguments for claiming an error-corrected and privacy-amplified secret key rate of the final key of more than 0.5 bit per photon.

\subsection{Experiment}

\subsubsection{Encoding}

We implement a high-dimensional version of the BB84 protocol using the $x, y$ spatial translation of single photons to encode information [55, 134]. A detailed description of our setup is given in section 5.2.2. The working principle of the protocol is illustrated in Fig. 5.1. We define detection areas on the twodimensional plane representing the symbols of our alphabet. The detection areas span $10 \times 10$ pixels on our single-photon sensitive detector. All the areas are arranged in a two-dimensional grid of $32 \times 32$ symbols. In this way, we are able to encode $d=32^{2}=1024$ symbols in total, which allows a theoretical maximum of $I_{\max }=10$ bit encoded in a single photon. The protocol requires a second, mutually unbiased, basis to guarantee that a measurement in the wrong basis yields no information. In general it is always possible to use a Fourier transform to form this second basis. In optics, a single lens performs this task. Alice and Bob both switch between an imaging path and a Fourier path. Only two of the four possible combinations will reveal all the information that Alice encoded to Bob. From the remaining two, no information can be extracted by Bob.

\subsubsection{Setup}

Our setup consists of a single-photon source, a spatial encoder, optics, the freespace quantum channel between Alice and Bob and finally a decoder together with a single-photon-sensitive position-reading detector on Bob's side.

The setup is schematically shown in Fig. 5.2. We use spontaneous parametric down-conversion (SPDC) as a source of photon pairs, here called signal and herald. The wavelength of the generated photons is $790 \mathrm{~nm}$ with a heralded 


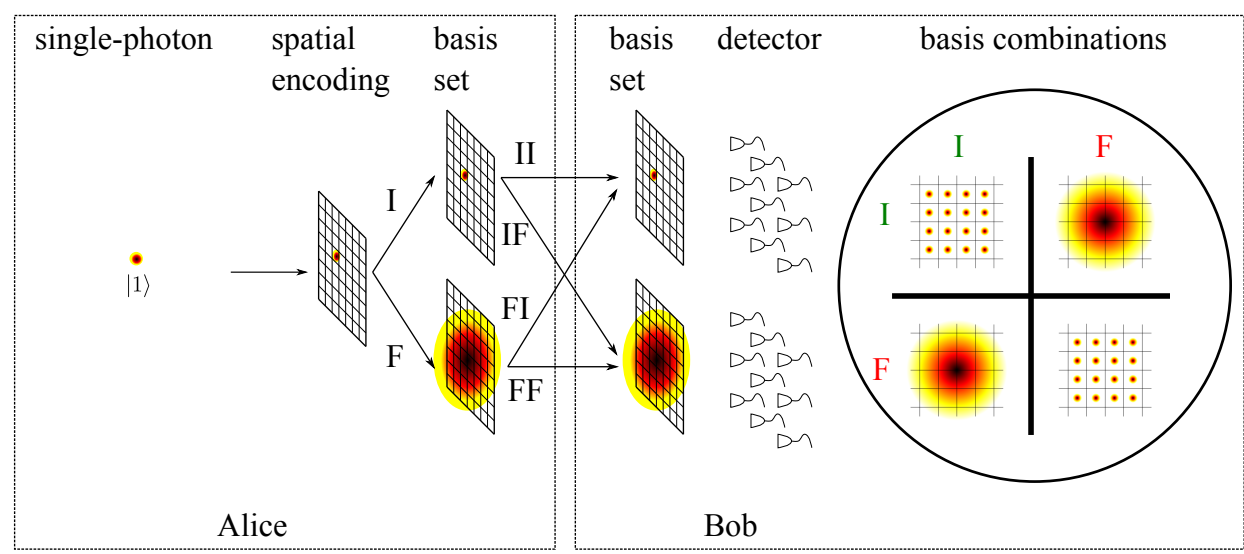

Figure 5.1 Illustration of our spatial encoding and decoding scheme and possible basis choices therein. The single-photon state $|1\rangle$ is encoded in the $\mathrm{x}, \mathrm{y}-$ translation basis formed by shifting a focus over a two-dimensional grid. Alice can send an image of the focus (I) or its Fourier transform (F) to Bob. Bob randomly switches between the two bases I and F. Only if the two bases are compatible (II or FF), the information encoded by Alice can be read out by Bob. In the two other cases (IF or FI), the information is low. Just like in BB84, a public channel is used for post processing including revealing the bases choices, detection of eavesdropping, error correction and privacy amplification.

single-photon count rate of $280 \mathrm{kHz}$ and a coincidence probability of $10 \%$ measured with two avalanche photodiods (APDs). The photon in the herald arm is detected by one of the APDs and signals a successful photon pair creation. The signal photon is sent through a $77 \mathrm{~m}$ single-mode fiber (SMF) to add an optical delay. The fiber output coupler (Thorlabs F220FC-780) and a $75 \mathrm{~mm}$ lens together with a $300 \mathrm{~mm}$ lens expand the light to a collimated beam of $8.4 \mathrm{~mm}$ FWHM to match the size of the spatial light modulator (SLM). We use a phaseonly liquid-crystal SLM to write a blazed grating in the phase of the wavefront. A $500 \mathrm{~mm}$ lens focuses the light to distinct $x, y$ positions in its focal plane. With a half-wave plate and a polarizing beam splitter (PBS), Alice can switch between the two mutually unbiased bases. One basis is designed for imaging the light in a $4 \mathrm{f}$ configuration with two $125 \mathrm{~mm}$ lenses. The other one performs a Fourier transform with a single lens with twice the focal length. In case the basis choice needs to be hidden from an eavesdropper, a second half-wave plate can be put after Alice's last PBS to counteract the polarization rotation of the first halfwave plate. After being transmitted via the quantum channel with two lenses $(f=50 \mathrm{~mm})$, the photons are randomly split by a beam splitter (BS) and are again guided through an imaging or a Fourier transform path for decoding. The 


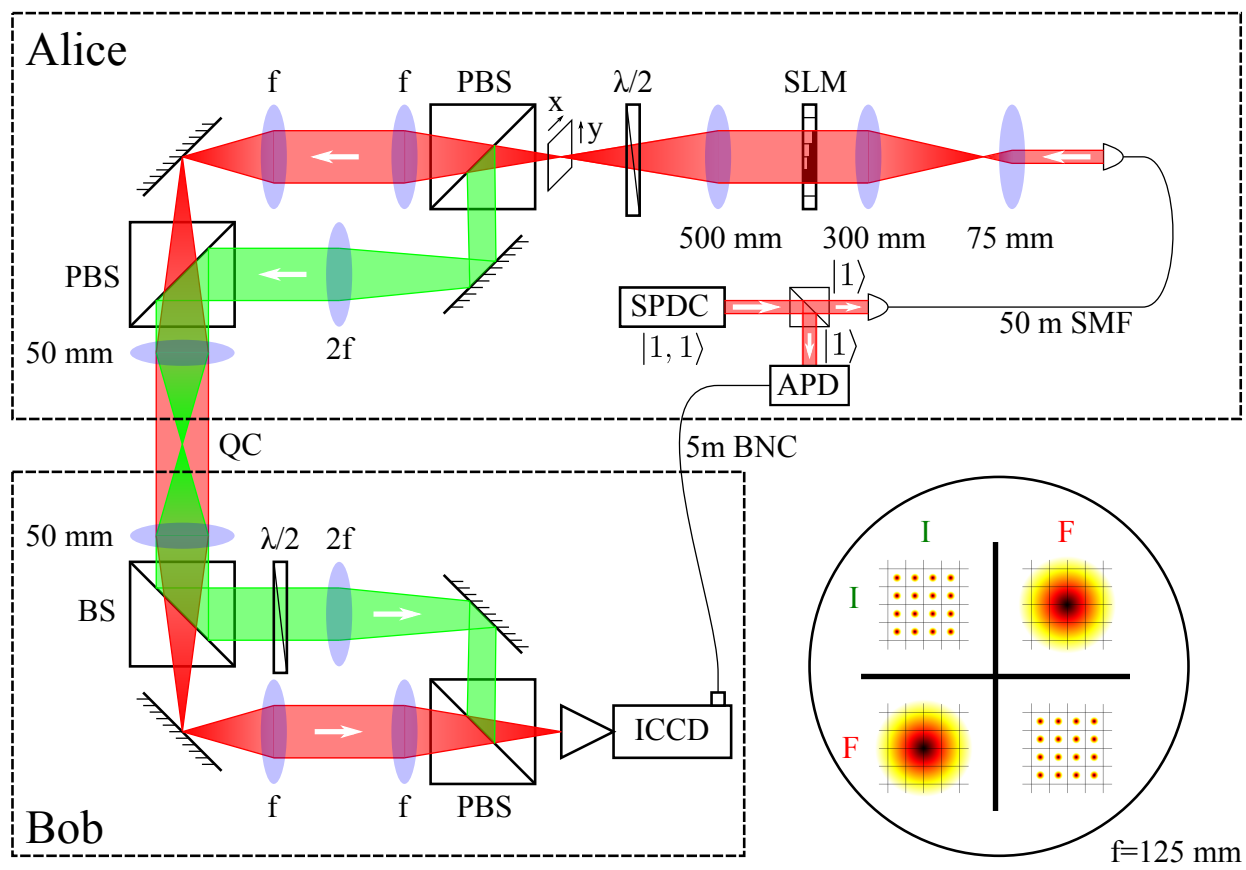

Figure 5.2 Schematic representation of the setup. We generate photon pairs at $790 \mathrm{~nm}$ by spontaneous parametric down-conversion (SPDC). One of the photons is coupled into a single-mode fiber (SMF) and the other is sent to an avalanche photodiode (APD) and used to trigger the camera. Information is encoded into the signal photon by translating the $\mathrm{x}$ and $\mathrm{y}$ position of the focus with a spatial light modulator (SLM) and a $500 \mathrm{~mm}$ lens. Alice chooses between two paths with a half-wave plate and a polarizing beam splitter (PBS), one (green) with a single lens (2f) and one (red) with two lenses (f). After the two beams are merged again by a second PBS, the light is guided through the quantum channel (QC) with two $50 \mathrm{~mm}$ lenses. Bob has the same set of lenses as Alice. His two paths are chosen randomly by a beam splitter (BS). The additional half-wave plate makes sure that all the light is directed to the camera (ICCD) by the last PBS.

half-wave plate in the Fourier path ensures that all the light is reflected to the intensified charged-coupled detector (ICCD). The ICCD is triggered via a $5 \mathrm{~m}$ $\mathrm{BNC}$ cable to match the detection window of the camera to the arrival of the signal photon. 


\subsubsection{Detection}

To detect the photons in a two-dimensional grid, we use an ICCD (Lambert HICAM 500S). It consists of an intensifier stage fiber-coupled to a CMOS camera of $1280 \times 1024$ pixels with a pixel size of $14 \times 14 \mu \mathrm{m}$. The photocathode of the ICCD acts as a gate and is triggered by the herald photons at $280 \mathrm{kHz}$. The delay between the trigger signal and the signal photon was measured to be $91 \mathrm{~ns}$. The gate width of the intensifier is $5 \mathrm{~ns}$. The CMOS camera is read out with 500 frames per second. The variance of the read-out noise of the CMOS is 0.4 counts and a threshold of 5 counts is set to filter the readout noise from the data. Moreover, a threshold on the size and intensity of detection events is set to between 2 and 10 pixels and between 1 and 60 counts, respectively, to remove unwanted spurious ion events.

\subsubsection{Classical light measurements}

Before the quantum measurements, the setup is characterized by an Obis laser with $785 \mathrm{~nm}$ wavelength. As a detector, a Stingray F-145 B is used. This allows to analyze the focus in the $x, y$-plane. The detector is divided in areas of $10 \times 10$ pixels. The SLM is programmed to focus the light on the middle of these areas, scanning over $78 \times 63=4914$ different positions in total. The intensity of the laser is kept constant, while one image is taken for each of the 4914 positions in all 4 possible basis configurations of the setup. In the two compatible basis, a $2 D$ Gaussian function

$$
G(x, y)=A \exp \left(-\left(\frac{\left(x-x_{0}\right)^{2}}{2 \Delta x^{2}}+\frac{\left(y-y_{0}\right)^{2}}{2 \Delta y^{2}}\right)\right)
$$

is fitted to the intensity profile on Bob's camera to extract the focus position $\left(x_{0}, y_{0}\right)$, the amplitude $A$ and the widths $\Delta x$ and $\Delta y$ in $x$ and $y$ direction. The results of this measurement are presented in figure 5.3. It shows the widths in $x$ and $y$ as well as their mean values. Overall, the periodicity in the graphs reflects the 78 columns of the map. At the edges of the map, the blazed grating on the SLM has its smallest periodicity, which results in a broader first diffraction order. The average widths in $x$ direction of $19.2 \pm 4.9 \mu \mathrm{m}$ in II and $22.8 \pm 3.9 \mu \mathrm{m}$ in FF is smaller than the one in $y$ direction of $20.7 \pm 0.7 \mu \mathrm{m}$ in II an $24.2 \pm 0.4 \mu \mathrm{m}$ in FF. The reason for that is the rectangular shape of the SLM window, which causes a smaller width in the $x$ direction. The asymmetry in the number of columns and rows of the map results in a larger standard variance of the $x$ widths. 


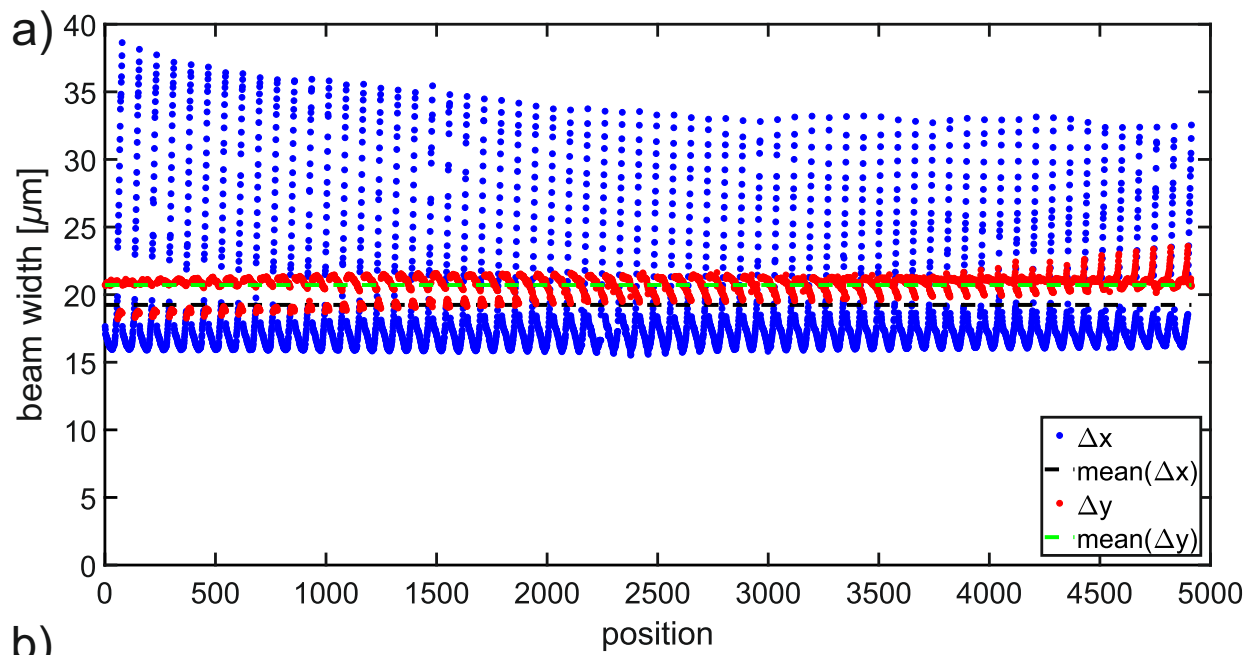

b)

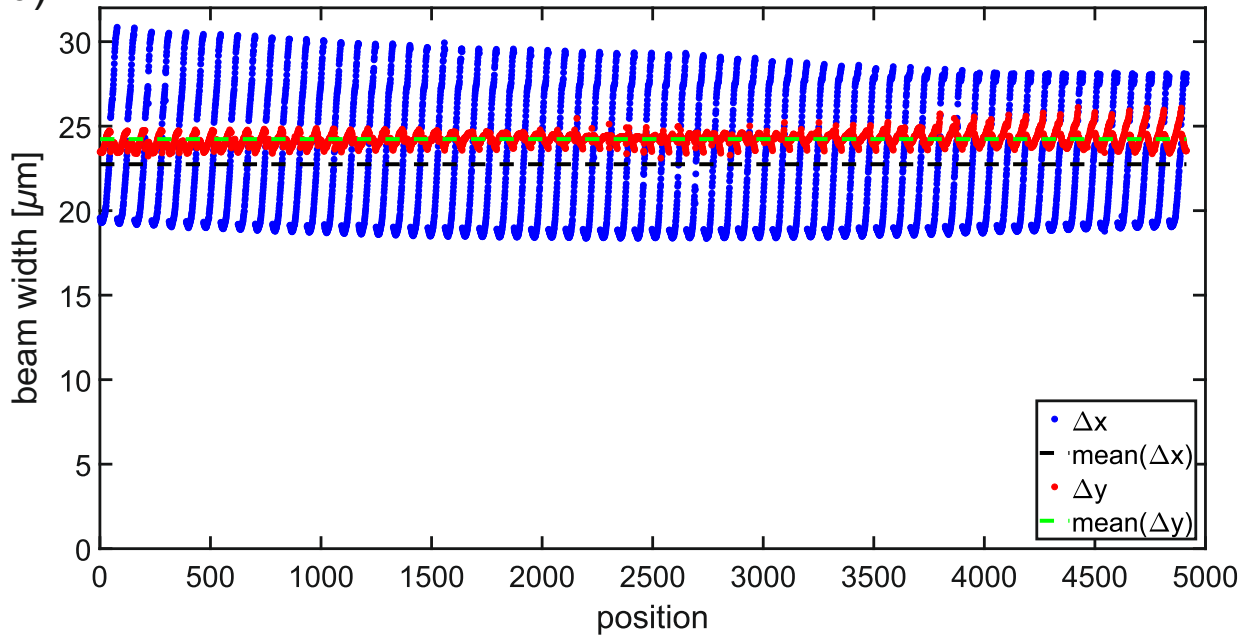

Figure 5.3 The beam widths of the focus on Bob's camera in $x$ (blue) and $y$ (red) direction in a) II-configuration and b) FF-configuration. The mean values are plotted in dashed lines. 

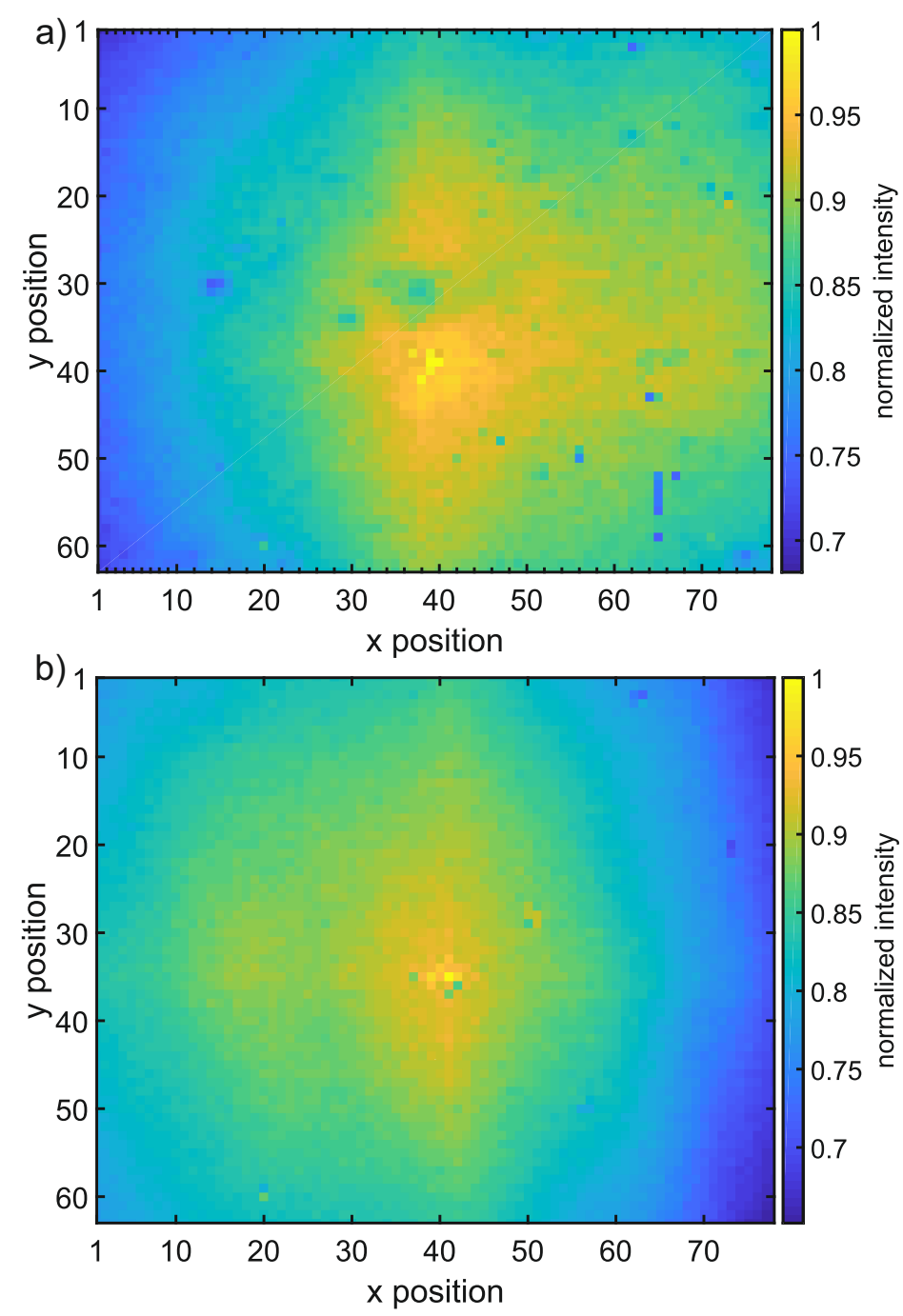

Figure 5.4 The normalized intensity scanned over all 4914 positions in a) IIconfiguration and b) FF-configuration.

Figure 5.4 shows the normalized intensity calculated from the parameters of the $2 D$ Gaussian fit function. In the II-configuration, the normalized intensity has a value of $86 \pm 5 \%$. In FF-configuration, the average value is $83 \pm 5 \%$. As apparent from the figure the intensity profile is reasonable flat, except for the two edges in the $x$ direction. In order to maintain a flat intensity profile during the single-photon experiment, the center region corresponding to $70 \times 70$ areas of this map was used for the QKD experiment. 

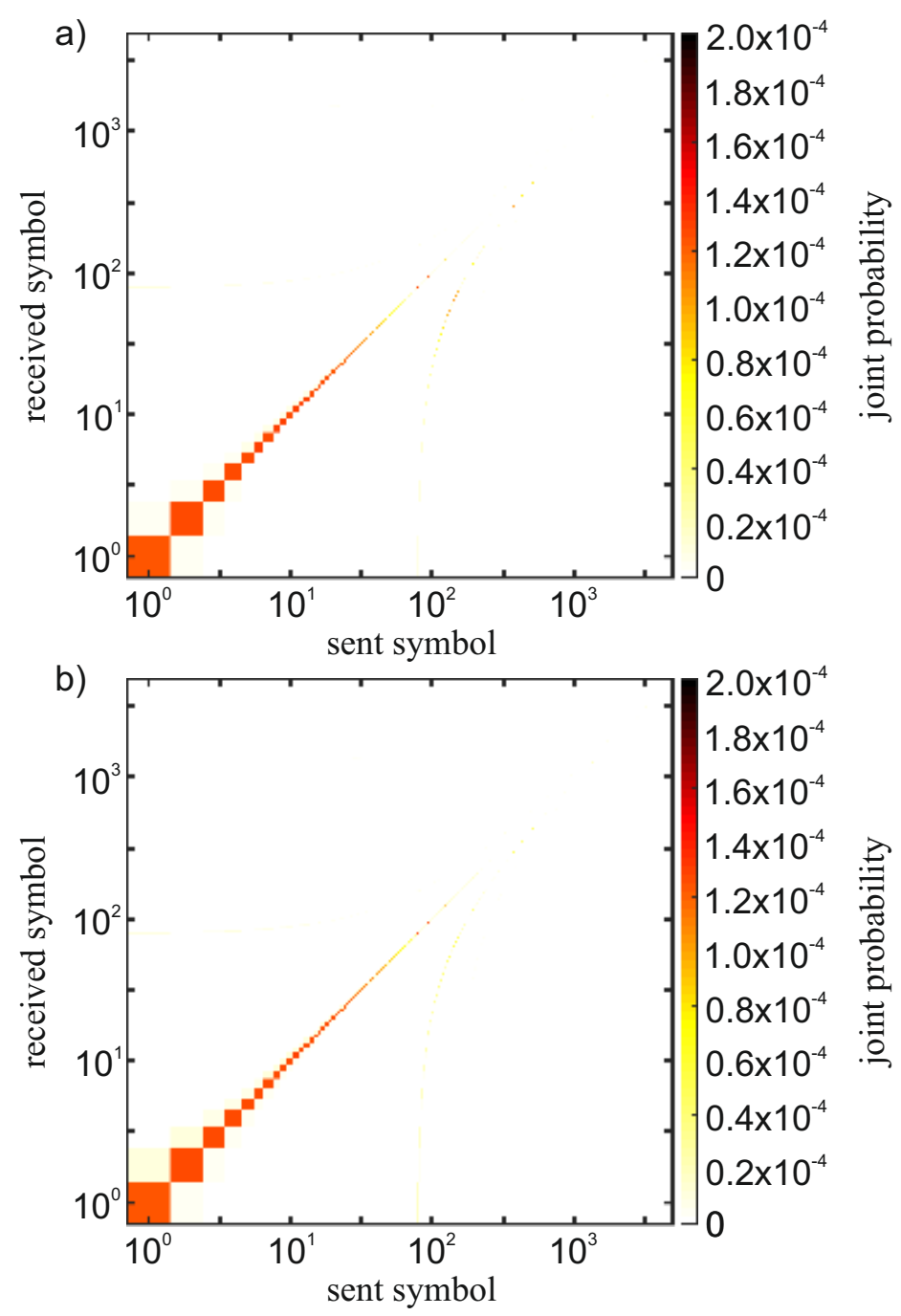

Figure 5.5 A log-log plot of the joint probability of the sent versus the received symbol in a) II-configuration and b) FF-configuration.

In figure 5.5, the joint probability distribution of the sent versus the received alphabet is plotted in both compatible bases. The mutual information is calculated with equation (2.7). This sampled mutual information is 10.06 bit in II and 9.56 bit in FF-configuration. Using the fitted data for the width of the focus, the upper limit can be calculated as shown in section 3.2.2. The number of dark counts is set to 0 and a the detector efficiency is set to 1 . Overall the upper limit to the mutual information is is 11.08 bit in II and 10.66 bit in FF configuration. The deviation from this limit is caused by experimental imperfections like 
pointing errors and the changes of the focus width due to the lower diffraction efficiency of the high spatial frequency blazed gratings on the SLM.

\subsubsection{Timing and Losses}

To reduce the dark counts of the detector, the detection window must be chosen as short as possible. The minimum gate width of the gate generator is $5 \mathrm{~ns}$. This gate window must be matched to the arrival of the signal photon. The SPDC source allows to use one of the photons as a herald of the other. Hence, this herald photon is detected with an APD and the electric pulse is directed to the gate generator. An additional $50 \mathrm{~ns}$ single mode fiber is used as optical delay for the signal photon. The SLM is set to a constant phase and the II-configuration is used. The ICCD is set to a repetition rate of $500 \mathrm{fps}$. The detector counts in a $20 \times 20$ pixels area around the focus are summed up over 1000 images. The delay is changed in steps of $1 \mathrm{~ns}$. The result of this measurement is shown in figure 5.6. The delay was set to $91 \mathrm{~ns}$, to match the detection to with the arrival of the signal photon.

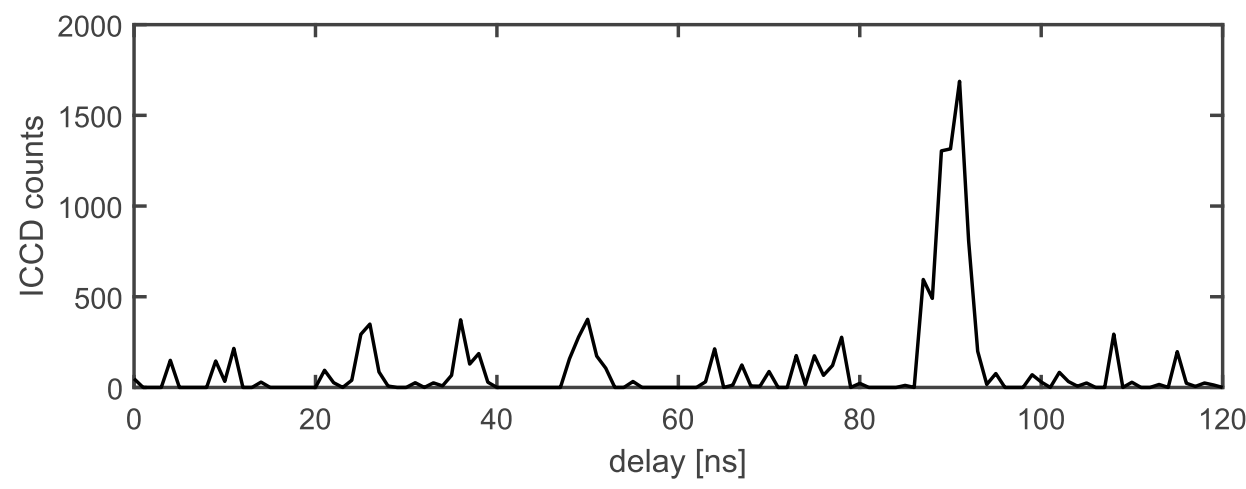

Figure 5.6 The counts at the focus position versus the delay set at the delay generator.

The losses for all four basis combinations have been measured with a power meter and coherent light. The measurements are taken after the $50 \mathrm{~m}$ fiber collimator and in front of the detector. The losses in II and FF are $17.5 \%$ and $17.0 \%$, respectively. In FI the losses are $18.5 \%$ and $18.7 \%$ in IF configuration. The average losses are $18.2 \%$. 


\subsection{Results}

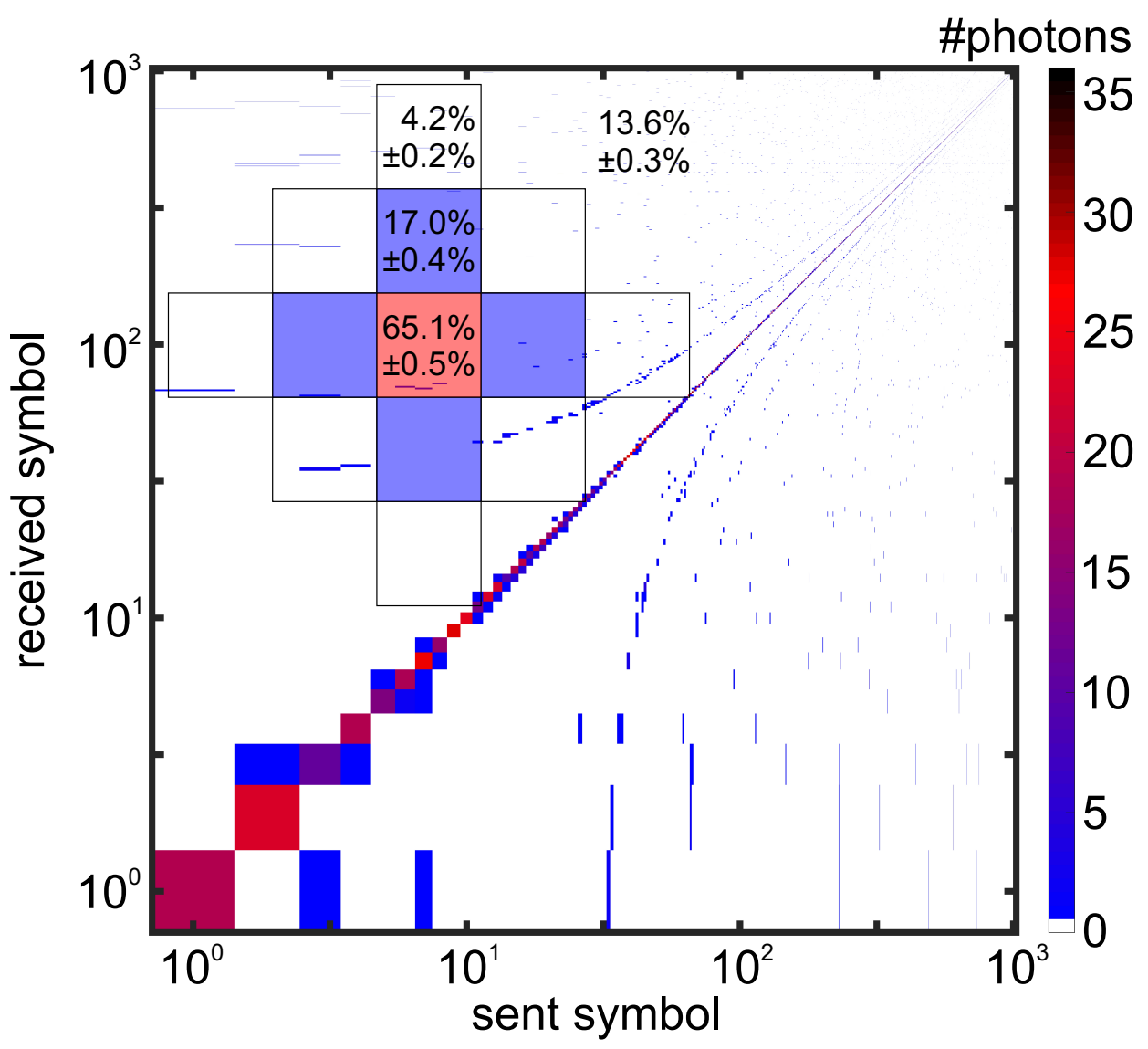

Figure 5.7 Correlation map of the received symbol index versus the sent symbol index on a logarithmic scale in II-configuration. The diagonal line indicates a strong correlation between the sent and the received symbols. In the top left corner, the hit distribution to the nearest and next-nearest neighbor symbols is shown. The events are visible in the correlation map as the lines shifted by the number of columns of the map. The average hit probability of the target area (red) is $P_{0}$, that of the four nearest neighbors (blue) is $P_{1}$, that of the eight next nearest neighbors (white) $P_{2}$. The remaining probability is summed in $P_{\text {rest }}=1-P_{0}-P_{1}-P_{2}$.

First, we characterize the information content of the transmission from Alice to Bob. For this purpose, we analyze the two compatible bases choices of Alice and 
Bob (II and FF). Alice sends each symbol $x$ out of her alphabet $X$ individually, while Bob receives the symbol $y$ out of the alphabet $Y .1000$ images per symbol are recorded on Bob's side. This step is performed for both compatible bases. In Fig. 5.7 the number of photons detected per symbol is shown in a log-log plot. In this figure, the joint probability function $p(x, y)$ is sampled, where $x$ is an element of the sent alphabet $X$ and $y$ from the received alphabet $Y$. We quantify the shared information between Alice and Bob by the mutual information [27]

$$
I(X ; Y)=\sum_{x \in X, y \in Y} p(x, y) \log _{2}\left(\frac{p(x, y)}{p(x) p(y)}\right),
$$

where $p(y)$ is the probability to measure symbol $y$ and $p(x)$ the probability of a sent symbol $x$. The maximum information Alice can send per symbol is $I$ (Alice) $=10$ bit. Due to noise in the channel and in the detection and imperfections in the information encoding, the shared information between Alice and Bob is smaller. For the II and FF basis configuration, we calculated the sampled mutual information to be $I(X: Y)_{\mathrm{II}}=8.3$ bit and $I(X: Y)_{\mathrm{FF}}=8.1 \mathrm{bit}$, respectively. The two main contributions to the noise are the cross talk to the neighboring detection areas, which was $13.3 \%$ and the dark counts of the detector which was $13.8 \%$.

Despite considerable experimental efforts, the probabilities used in the calculation of the mutual information are under-sampled with an average of 73 detection events per symbol. This means that neighboring pixel cross-talk events are not accurately sampled, a problem that gets increasingly severe for larger alphabets. If Eve uses an optimal cloner [73], the minimum fidelity for cloning-based individual attacks is 51.6\% [44]. Introducing the average symbol hit probability $F$, the mutual information in equation (5.2) can be simplified to

$$
\begin{aligned}
I(F) & =\log _{2}(d)+F \log _{2}(F) \\
& +(1-F) \log _{2}\left(\frac{1-F}{d-1}\right),
\end{aligned}
$$

where $d$ is the dimensionality of the basis. In our experiment, $F=68.7 \%$. Since a large portion of the photons hits the neighboring areas, equation (5.3) is an underestimate and can be refined by adding the hit probabilities $P_{0}, P_{1}, P_{2}$ and $P_{\text {rest }}$ defined in the top left corner of Fig. 5.7. We assume the values $P_{0}, P_{1}, P_{2}$ and $P_{\text {rest }}$ are equal for each symbol and derive

$$
\begin{aligned}
I_{\mathrm{AB}} & =\log _{2}(d) \\
& +P_{0} \log _{2}\left(P_{0}\right)+P_{1} \log _{2}\left(\frac{P_{1}}{4}\right) \\
& +P_{2} \log _{2}\left(\frac{P_{2}}{8}\right)+P_{\text {rest }} \log _{2}\left(\frac{P_{\text {rest }}}{d-13}\right) .
\end{aligned}
$$


The resulting mutual information is $6.75 \pm 0.08$ bit in the II-configuration and $7.03 \pm 0.04$ bit in the FF-configuration.

\subsection{Discussion}

One important criterion for the security of QKD is that the basis choice of Alice remains hidden from Eve. We use Gaussian optics in our setup, which can be corrected for in post processing. As a result, we have Gaussian foci with finite width in the focus plane. The Fourier transform of a Gaussian function is another Gaussian function as seen in Fig. 5.8. In the Fourier basis, the probability to detect a photon is higher in the center than at the edges. If Alice sends all symbols of her alphabet with the same probability, Eve could therefore make a reasonable guess which basis is used. A photon detection at the edge of the detector is more likely to have been sent in the imaging basis, while a detection in the center is more likely in the Fourier basis. We measured the photon hit distribution for the two incompatible bases choices IF and FI with the same parameters as in the compatible case. In Fig. 5.8, the distribution is shown with a Gaussian fit. The width in the columns is $89.9 \pm 1.7$ pixel and $106.7 \pm 1.9$ pixel in the rows together with $96.3 \pm 2.5$ pixel and $102 \pm 3$ pixel in the FI configuration. To close the leak, Alice can adjust her send probability $p(k)$ to this Gaussian distribution. As a result, the information sent by Alice $-\sum_{k=0}^{d-1} p(k) \log _{2}(p(k))$ reduces from 10 bit to $I$ (Alice) $)_{\mathrm{II}}=9.4$ bit and $I$ (Alice $)_{\mathrm{FF}}=9.4$ bit. Consequently, the sampled mutual information with the hidden basis drops to [55]

$$
\begin{aligned}
I_{\mathrm{hb}} & =I(\text { Alice })+\sum_{k=0}^{d-1} p(k) F_{\mathrm{eff}} \log _{2}\left(F_{\mathrm{eff}}\right) \\
& +\sum_{k=0}^{d-1} \sum_{j=0, j \neq k}^{d-1} \frac{p(k)\left(1-F_{\mathrm{eff}}\right) p(j)}{1-p(k)} \log _{2}\left(\frac{\left(1-F_{\mathrm{eff}}\right) p(j)}{1-p(k)}\right)
\end{aligned}
$$

with the effective fidelity $F_{\text {eff }}$ defined by $I\left(F_{\text {eff }}\right)=I_{\mathrm{AB}}$ in combining equation (5.3) and (5.4). This results in $\left(F_{\text {eff }}\right)_{\mathrm{II}}=75.5 \%$ and $\left(F_{\text {eff }}\right)_{\mathrm{FF}}=77.9 \%$ leading to $\left(I_{\mathrm{hb}}\right)_{\mathrm{II}}=6.3$ bit and $\left(I_{\mathrm{hb}}\right)_{\mathrm{FF}}=6.6$ bit. 


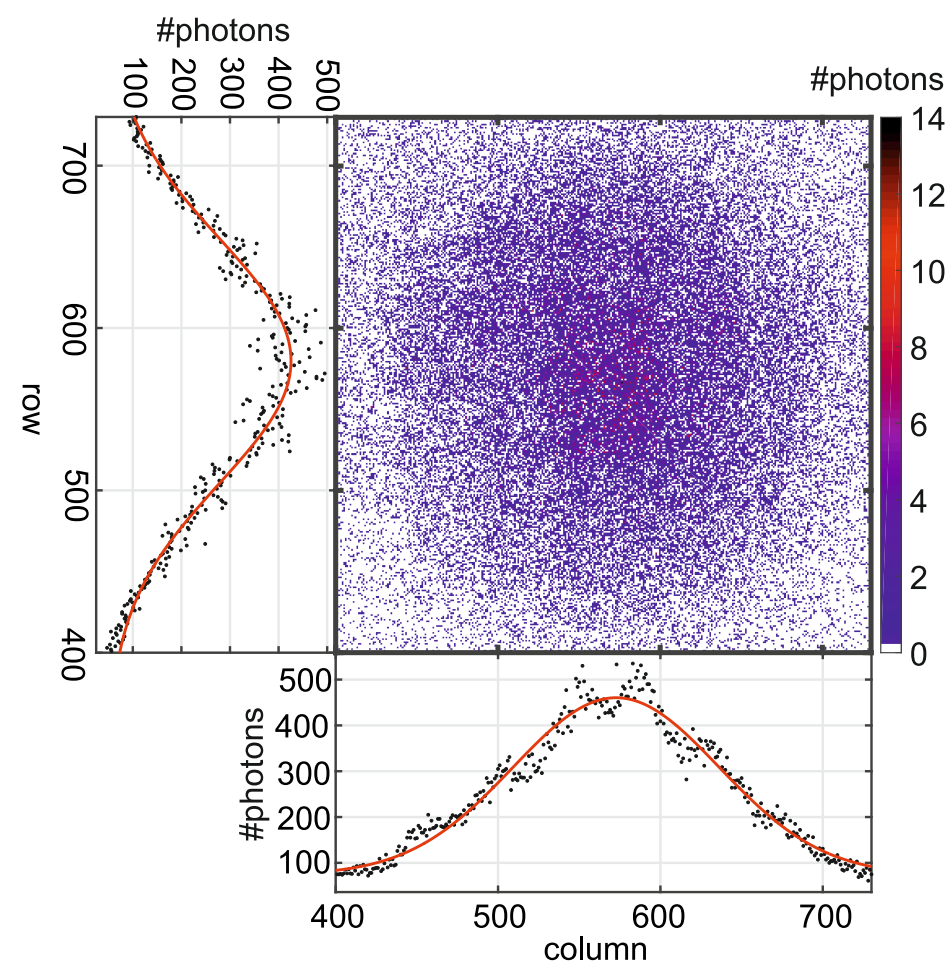

Figure 5.8 Image integrated over all Bob's 1024 different spot positions in the IF configuration. Projections of the signal are shown left of and below the plot by summing over the columns (lower panel) and rows (left panel). The photon counts follow a Gaussian spatial distribution as is evident from the red fit curves.

In postprocessing via the public channel, Alice and Bob reveal their basis choice. They only keep the measurement results in the two compatible bases, which bisects the key length. To check for eavesdropping, the quantum bit error rate of this sifted key needs to be calculated. We used the Gray code [107] to encode the $\mathrm{x}$ and $\mathrm{y}$ position of the symbol in a bit string. In this way we reduce the bit error rate, since $31.3 \%$ of the error is due to crosstalk to neighboring symbols. In the Gray code, neighboring symbols have a Hamming distance of only 1 . We calculated the averaged quantum bit error rate over all symbols to be $Q_{\mathrm{II}}=7.8 \%$ for the II-configuration and $Q_{\mathrm{FF}}=7.4 \%$ for the FF-configuration. We calculated the secret fraction of the key in case of intercept-resend attacks and infinite key length in the supplementary information. 


\subsubsection{Intercept-resend attack}

For the security of the protocol, we need to ensure that the information Eve can gain from intercepting the communication is lower than the mutual information between sender and receiver. Assuming an intercept-resend attack, the information Eve can learn is $I$ (Eve) $=\frac{\eta}{2} I$ (Alice) with $\eta$ the fraction of intercepted photons and $I$ (Alice) the sent information of Alice. Averaged over the compatible bases, we find $I$ (Alice) $=9.4$ bit. An eavesdropper can extract a maximum of $I$ (Eve) $=\eta 5.72$ bit. Therefore, just as in the case of the original BB84, information gain is only possible at the expense of disturbing the signal [27]. An eavesdropper will be recognized by increasing the error rate of the key generated by Alice and Bob. Alice and Bob will have to compare a random part of their key to decide if they have been eavesdropped. Intercepting a fraction of $\eta$ photons, an attacker introduces an error of

$$
E_{\mathrm{Eve}}=\frac{\eta}{2} \frac{d-1}{d}
$$

where $d$ is the number of symbols. To calculate the quantum bit error rate of the sifted key, we used the Gray code [107] to encode the $x$ and y position of the symbol in a bit string. In this way, we reduce the bit error rate, since $31.3 \%$ of the error is due to crosstalk to neighboring symbols. In the Gray code, neighboring symbols have a minimum hamming distance of 1 . We calculated the averaged quantum bit error rate over all symbols to be $Q_{\mathrm{II}}=0.078$ with a standard deviation of $\Delta Q_{\mathrm{II}}=0.042$ for II-configuration and $Q_{\mathrm{FF}}=0.074$ and $\Delta Q_{\mathrm{FF}}=0.029$ for FF-configuration. Assuming Alice and Bob set their threshold to detect eavesdropping to a bit error rate of $Q+\sigma$, where $Q$ is the averaged quantum bit error rate. In this case Eve could only intercept a fraction of $\eta$ of the photons.

\subsubsection{Basis guess fidelity}

In practical QKD, Alice's basis choice could leak to an eavesdropper via side channels or imperfect encoding. To include this into the model, we added a guess fidelity of $\epsilon$. Eve can not guess the basis if $\epsilon=0$, while $\epsilon=1$ means that Eve knows Alice's basis choice. In our experiment, we measured $\epsilon \sim 0.15$ by a correlation measurement performed with classical light. Eve can then extract

$$
I(\text { Eve })=\frac{\eta}{2}(1+\epsilon) I(\text { Alice })
$$

from the information Alice sends. Thereby she adds an additional error of

$$
Q_{\text {Eve }}=\frac{1}{2}(1-\epsilon) \frac{d-1}{d} .
$$

To detect eavesdropping, Alice and Bob must set an error threshold $\sigma$. The error rate introduced by Eve's perturbation of the quantum channel has to be lower 
than this threshold to stay unnoticed. The quantum bit error rate including an eavesdropper is

$$
\begin{aligned}
Q_{\text {Total }} & =(1-\eta) Q+\eta\left(Q+(1-Q) Q_{\text {Eve }}\right) \\
& =Q+(1-Q) \eta Q_{\text {Eve }} .
\end{aligned}
$$

From the relation

$$
Q_{\text {Total }} \leq Q+\sigma,
$$

the maximum fraction of intercepted photons is

$$
\eta_{\max }=\frac{\sigma}{(1-Q) Q_{\mathrm{Eve}}},
$$

which depends on the fidelity to guess the correct basis $\epsilon$ and the threshold $\sigma$. The minimum fidelity of Bob reduces to

$$
F_{\text {Total }}=F\left(1-\eta Q_{\text {Eve }}\right) .
$$

Now it is possible to calculate the distance of information between Bob and Eve, which is a measure for the secure key rate. The information distance is defined as

$$
\delta=I_{\mathrm{AB}}-I(\text { Eve })
$$

The amount of information Bob receives depends on the amount of information Alice transmits and on the channel noise. Therefore

$$
I(\text { Bob })=Q_{\text {Total }} I \text { (Alice) }
$$

Combining equation (5.7) and (5.15), the information distance can be written as

$$
\delta=\left[\left(1-Q_{\text {Total }}\right)-\frac{\eta}{2}(1+\epsilon)\right] I(\text { Alice }) .
$$

By substituting $\eta_{\max }$ from equation (5.12) in this expression, the minimum information distance can be plotted as a function of $\epsilon$ and $\sigma$ in figure 5.9. Compared to equation (5.17) the prefactor $n / N$ does not appear, since already a small fraction of the key is enough for parameter estimation. Moreover, the error correction and parameter estimation as well as the uncertainties about Eve's entropies lower the secret fraction. 


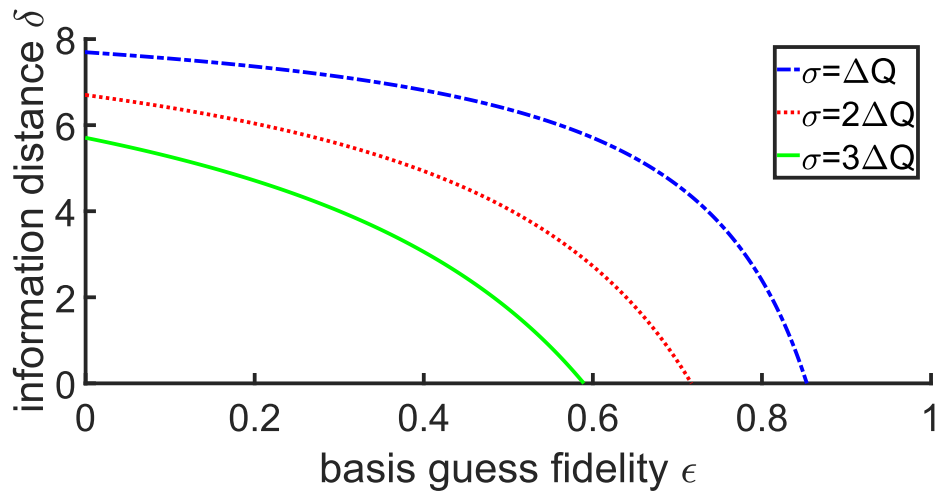

Figure 5.9 The minimum secret information distance $\delta$ against the basis guess fidelity $\epsilon$ for three different thresholds $\sigma$.

The information distance $\delta$ becomes monotonically larger with decreasing threshold $\sigma$, but becomes smaller with increasing $\epsilon$, as visible in Fig. 5.9. If Eve knows Alice's basis choice $(\epsilon=1)$, her measurements will no longer add noise, which allows her to intercept the quantum communication without being detected. In comparison to the finite-key-length secret fraction in the case of collective attacks, the values the minimum secret information $\delta$ is larger.

\subsubsection{Finite key bound}

For security arguments against collective attacks, we used finite key considerations given in [89-91]. In the case of a finite key length, $N<\infty$, failure probabilities in each step of postprocessing need to be considered. After sifting the key and removing the incompatible basis choices of Alice and Bob, the key length bisects. From this key length, half the symbols are used to check for the presence of an eavesdropper. The next step is error correction to achieve an error-free key. Due to the finite key length the error correction has a finite failure probability and not all errors can be removed. Assuming a two-way cascade code [80], this failure probability is $\epsilon_{\mathrm{EC}} \sim 10^{-5}[81,135]$ in case of a $8 \%$ bit error rate. To limit the maximum information of Eve, a privacy amplification step needs to be performed. With average bound privacy amplification [82, 136], the information of Eve can be bound to $3 \times 10^{-10}$ bit with a failure probability of $\epsilon_{\mathrm{PA}}=2^{-15}$. In this case, the lower bound for the secret key rate per photon of a $\epsilon=10^{-5}$ secure key is given by [89-91]

$$
r_{N}=\frac{n}{N}\left(I_{\mathrm{AB}}-I(\text { Eve })-\frac{1}{n} \log _{2}\left(\frac{2}{\epsilon_{\mathrm{EC}}}\right)-\frac{2}{n} \log _{2}\left(\frac{1}{\epsilon_{\mathrm{PA}}}\right)\right) .
$$

We neglect the failure probability introduced by smoothening the entropies. If both bases are used with the same probability, $n=0.25 \mathrm{~N}$ symbols can be used 
to create a key. $I_{\mathrm{AB}}$ is defined in equation (5.4) and is the mutual information between Alice and Bob and

$$
I(\text { Eve })=-(1-F) \log _{2}\left(\frac{1-F}{d-1}\right)-F \log _{2} F
$$

is Eve's information assuming all channel errors are attributed to the presence of an eavesdropper [44]. We assume the worst case values in parameter estimation and replace the fidelity $F$ by $F-\Delta F / \sqrt{n}$ to take the statistical fluctuations of the measured fidelity into account. The remaining terms in equation (5.17) are the influence of the failure probabilities on the secret key rate.

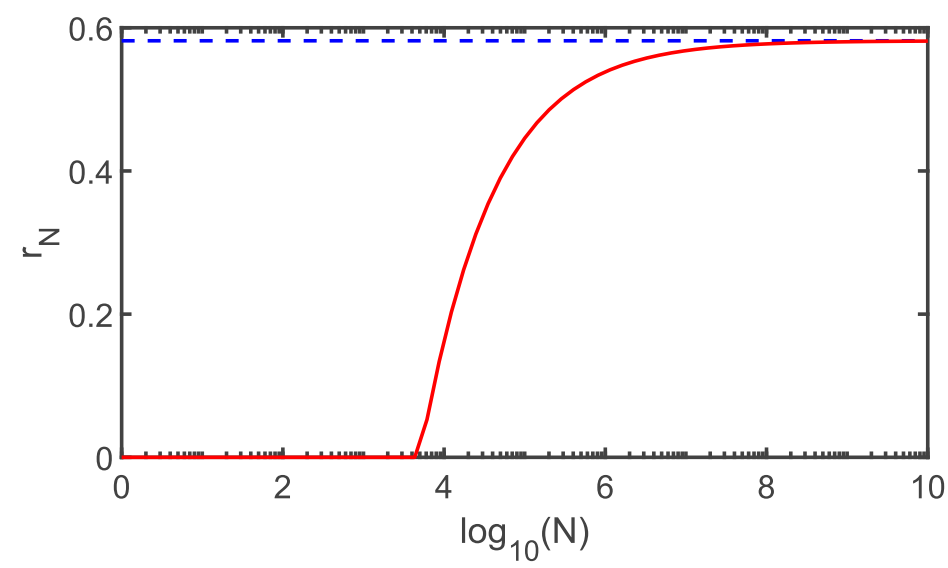

Figure 5.10 The lower bound of the secret key rate $r_{N}$ per detected photon as a function of the logarithm of the key length $N$ (red). The blue dashed line represents the asymptotic limit of infinite key length. The failure probabilities are $\epsilon_{\mathrm{EC}}=10^{-5}$ and $\epsilon_{\mathrm{PA}}=2^{-15}$. The quantum bit error rate is $Q=0.08$.

Figure 5.10 shows the minimum secret key rate as a function of the number of symbols. With increasing key length, the secret key rate approaches its asymptotic limit, which is the difference between the shared information between Alice and Bob and the information of Eve. As seen in the figure, we can establish a non-zero secret key rate starting from a key length $5 \cdot 10^{3}$ symbols. Assuming an SLM with a maximum frame rate of $60 \mathrm{fps}$, such a key can be generated in $\approx 3$ minutes. 


\subsection{Conclusion}

In this chapter, we experimentally demonstrate high-dimensional QKD using spatially encoded photons. We encode an alphabet of 1024 symbols and achieve a channel capacity of 7 bit per detected photon. We discuss a solution to hide Alice's basis choice from Eve. Taking error correction and privacy amplification into account for finite key length, we establish a secret key fraction of 0.5 bit per photon. For longer-distance communication, the combination of this work with multimode fibers [137] appears attractive. 


\section{Multimode Fiber Communication}

\subsection{Introduction}

Quantum communication aims to provide absolutely secure transmission of secret information. Existing methods encode symbols into single photons or coherent light with much less than one photon on average. Here we propose and demonstrate an original method that is fundamentally secure with weak coherent light even when several photons per symbol are used. Our method does not require a change of measurement basis and works with a high-dimensional alphabet in a simple practical setup. Moreover, it sends information in a deterministic way, allowing direct decoding at the receiver end. This feature makes the method suitable for Quantum Key Distribution (QKD) as well as Quantum Secure Direct Communication (QSDC). The key element of our method is the combination of wavefront shaping with a multimode fiber channel. Since it is based on an optical fiber, our method allows to naturally extend secure communication to larger distances.

The importance of secure communication is rapidly growing [138]. We use cryptography in everyday life often without noticing, for example, when we conduct financial transactions via the internet. The security of conventional cryptography is based on shared secret keys or on computational assumptions, such as the presumed hardness of factoring [9]. In practice, this means that it is vulnerable to unanticipated advances in hardware or algorithms. Quantum cryptography in theory provides unconditional secure communication, assuming only that an eavesdropper (Eve) is restricted by the laws of physics: the quantum no-cloning theorem forbids to replicate an unknown quantum state [16]. Indeed, the security of quantum cryptography requires quantum states of light [26]. For example, in the original and best-known Quantum Key Distribution method - BB84 proposed by Bennett and Brassard [13] - the security is based on the fact that the polarization of a single photon can be prepared and measured along well-defined directions. Key distribution methods do not by themselves communicate useful information. Such communication can follow only after the secure key is built up. Recently methods for Quantum Secure Direct Communication were proposed [139-144]. However, QSDC is more demanding on the security: the eavesdropper should not only be detected but also obtain blind results. It makes QSDC very complicated for practical realizations[145].

Nowadays, optical fibers are key elements of worldwide communication[146]. Single-mode fibers are widely used to transmit voice, television and internet data

The content of this chapter has been submitted for publication as: L. V. Amitonova, T. B. H. Tentrup, I. M. Vellekoop, and P. W. H. Pinkse, "Multimode-fiber-based high-dimensional quantum secure communication,". 
by encoding this information temporally into a single optical mode. In theory, multimode fibers could have a much higher bandwidth because they carry thousands or hundreds of thousands of orthogonal optical modes. Although short pieces of straight or slightly bent fiber are not truly random [147], in any realistic fiber, the modes are coupled, leading to an arbitrary mixing of the field amplitudes [148] and scrambling the information across the modes. Random mode mixing in multimode fibers can be utilized for secret key distribution that cannot be hacked a posteriori by analyzing stored tapped data [149]. On the other hand, this mixing can partially be undone by applying techniques from complex wavefront shaping, a method originally developed for precise light control through and in highly scattering materials [121, 150, 151]. Multimode optical fibers can now be used to transmit information in the spatial domain [152-154]. Recently methods have been proposed for high-speed[155], highresolution [156, 157] image transfer. Wavefront-shaping methods already have been employed for quantum-secure authentication [61].

Here, we introduce and experimentally demonstrate a new type of quantum communication method based on a high-dimensional spatial alphabet encoded into the guided modes of a multimode fiber. The multimode fiber combines features of a Physical Unclonable Function (PUF) [63, 64] with the inherent ability to generate secret keys by configuration changes. The no-cloning theorem forbids an attacker to fully characterize a light pulse containing fewer photons than the number of mixed fiber modes. The wavefront shaping technique allows Bob and only Bob to read the information. The multimode-fiber-based method doesn't require a quantum key sifting step and allows to decode the secure information instantaneously during the transmission, providing a unique platform for Quantum Secure Direct Communication between two parties. The fiber nature of the proposed method allows to extend our method to long distances. The experimental realization of the method provides security in a simple setup and real-life implementations, which makes commercial applications conceivable.

\subsection{Result}

Firstly, we briefly discuss the main idea of the method under ideal conditions assuming a perfect single-photon source and ideal wavefront shaping means (in phase and amplitude) since it is the simplest to understand conceptually. In the Methods section, we give a quantitative argument why the method is still secure with weak coherent light and imperfect wavefront shaping without any adaptation to the method or the setup. The experimental setup and illustration of the main principle are presented in Fig. 6.1. By controlling both the phase and amplitude patterns of the wavefront, it is possible to focus all light to a desired position behind the fiber [158, 159]. The calibration procedure to find this unique wavefront is described in details in the Methods section. To send 
a symbol, Alice prepares a single-photon quantum state with an appropriately phase-shaped and amplitude-shaped wavefront that leads to focusing light at the particular position on the fiber output facet. Throughout the fiber, the single photon will be present as a disordered superposition of almost all fiber modes. If a potential eavesdropper Eve would intercept the photon and determine its position, the photon collapses at a nearly random position on Eve's detector, which varies even between identical copies of the same symbol (see Fig. 6.1 'Eve'). Therefore, Eve will not be able to identify what symbol was sent: the information is scrambled. It is important to note here that even if Eve knows the exact optical transmission matrix of the fiber between Alice and Eve, she cannot identify what symbol was sent by observing a single photon on her camera since the symbols are not spatially localized (see Methods section for a derivation of the maximum entropy that Eve could possibly gain from a measurement). Only at Bob's end, the photon will be spatially localized at the desired target position, where each target position corresponds to a symbol from the alphabet (see Fig. 6.1 ' $\mathrm{Bob}^{\prime}$ ). Since here the photon is spatially localized, Bob can unambiguously identify what symbol was sent: the information is unlocked. The only way that Eve could recover the information sent by Alice would be to copy and physically reproduce all essential features of the fiber between Eve and Bob in some kind of passive optical system that would focus each symbol onto a different detector. We assume that the long multimode fiber is sufficiently complex and random to be physically unclonable, ruling out this type of attack. Moreover, we assume that Bob regularly reconfigures his part of the fiber in an unpredictable way. Without a physical copy of the fiber, Eve will always be limited to measurements that collapse the photon state to a random position, thereby destroying almost all encoded information (see section 6.3 for a detailed derivation).

\subsubsection{Experimental demonstration of secure communication}

We perform our experiments in imperfect 'real-life' conditions: with a weak coherent light source, in the presence of noise, and assuming only a moderate efficiency of wavefront shaping. First, the fiber is calibrated in its current configuration as described in detail in the Methods section. As a result, Alice and only Alice has information about the phase masks required for focusing light at the different positions on the fiber output facet and Bob and only Bob knows the positions corresponding to a particular symbol. The set of special superpositions of fiber modes that lead to light focused at desired points on Bob's side for a particular fiber configuration we will call basis. In our experiments, we use a fiber with an approximate number of 1500 modes $(N=1500$, see section 6.3) and the alphanumeric: 36-dimensional alphabet consisting of A-Z + 0-9 (case insensitive) symbols. The symbols are encoded into 36 different positions on the fiber output facet, as presented in faint green circles in Fig. 6.2(a). In the first set of experiments, we emulate the perfect single-photon source by taking 


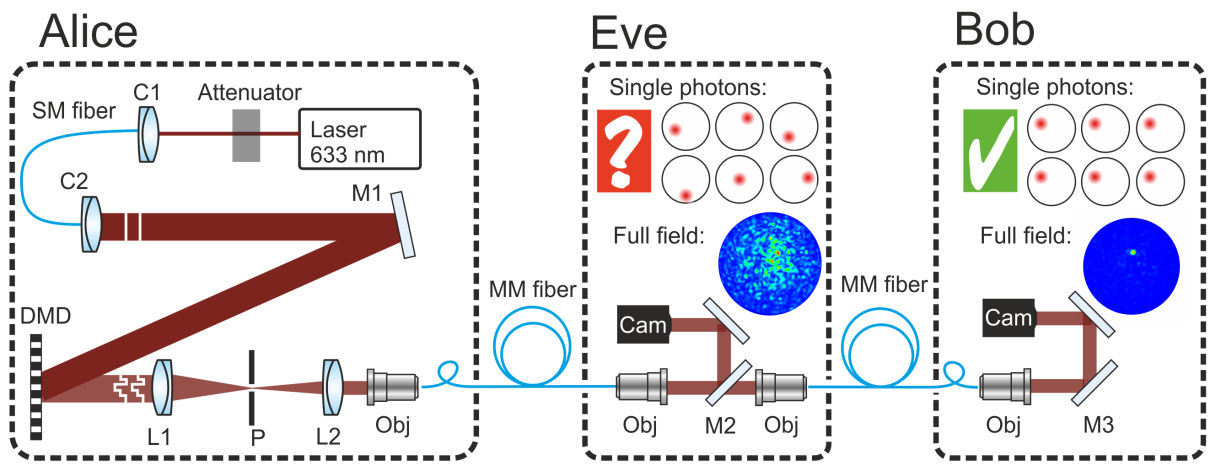

Figure 6.1 Schematic representation of the setup. Alice encodes a symbol by coupling light with an appropriate phase pattern to a multimode fiber. Bob receives a signal focused at a particular position on the fiber output facet (see 'Full field' on Bob's part). Bob can reconstruct the position even with a single detected photon (see 'Single photons' on Bob's part). With mirror M2 Eve can tap off the signal in the middle of the fiber. However, due to the mode-mixing nature of the multimode fiber, her intensity distribution is a complex interference pattern (see 'Full field' on Eve's part). The positions of her photon detections are nearly random and vary even for different realizations of the same symbol (see 'Single photons' on Eve's part). As a result, Eve cannot reconstruct the symbol that Alice sent. Abbreviations: MM fiber, multimode fiber; DMD, digital micromirror device; SM fiber, single mode fiber; $\mathrm{M}$, mirror; L, lens; Obj, objective; $\mathrm{P}$, pinhole; C, collimator; Cam, camera. 
into account only the frames with single-photon detection events. Snapshots of the spatial distributions of photons measured by Bob and by Eve are presented in Fig. 6.2(a) and 6.2(b), respectively. For clarity, we show frames which correspond to the fidelity of wavefront shaping $\alpha^{2}=0.6$ (for the definition of fidelity see section 6.3) and for only two different symbols sent by Alice by open and filled dots. Different colors represent 10 different repetitions of each of the two symbols. The dashed line shows the fiber core edge. In contrast to Eve, Bob clearly sees the correlation between different realizations of the same symbol. In case of a perfect single-photon light source, the probability for Bob to detect the photon in the correct position is equal to the fidelity of wavefront shaping that, as was shown before, can experimentally reach a theoretical maximum of $\pi / 4$ for phase-only wavefront shaping [152]. In contrast, different realizations of the same field pattern hardly correlate to each other on Eve's side (see Fig. 6.2(b)).

In the second set of experiments, we characterize the communication between Alice and Bob for low-fidelity wavefront shaping, $\alpha^{2}=0.1$ (see the Methods section), and weak coherent light source with an average number of photons per pulse $\bar{n}_{a}$ starting from 2. Alice sends each of the 36 symbols 200 times in random order. Each time Bob reads the signal on the fiber output and estimates what symbol was sent. In real life Bob cannot obtain all information that was sent because the fidelity of phase-only wavefront shaping is smaller than unity and because of other imperfections. He can use two main strategies. In the first strategy, Bob compares the number of photons in different predefined areas and chooses the one with the highest intensity. In the second strategy, Bob selects only those transmissions for which he is very sure what symbol was sent by accepting only the symbols with at least as many photons as a particular threshold. Whenever Bob has more information than Eve, the few-percent error rate can be corrected down to the standard $10^{-9}$ during the (classical) error correction step of the protocol [25].

We investigate the percentage of the correct reading and the rejection ratio for two different thresholds. Then we repeat the whole procedure for a different average number of photons per pulse. The results averaged over all 36 symbols are presented in Fig. 6.2(c), where the probability $p$ to detect the correct symbol versus the average number of photons in a pulse, $\bar{n}_{a}$, is plotted for different strategies of Bob: black, no threshold; red, two-photon threshold and blue, three-photon threshold. Dots represent the experimentally measured data. The black line shows the theoretical prediction from the following formula $p=e^{-\lambda_{1}} \sum_{k=0}^{\infty}[F(k-1)]^{S-1} \lambda_{1}^{k} / k$ !, where $F(k)=e^{-\lambda_{2}} \sum_{l=0}^{k} \lambda_{2}^{l} / l$ ! is the cumulative distribution function of the Poisson distribution, $\lambda_{1}$ is the average number of photons at the "correct" position, $\lambda_{2}$ is the average number of photons at the "wrong" position and $S$ is the total number of symbols. Values $\lambda_{1}=0.1 \bar{n}_{a}$ and $\lambda_{2}=0.005 \bar{n}_{a}$ were extracted from the experimental data. The blue and red lines represent the results of simulations of Bob's probability for a thresh- 

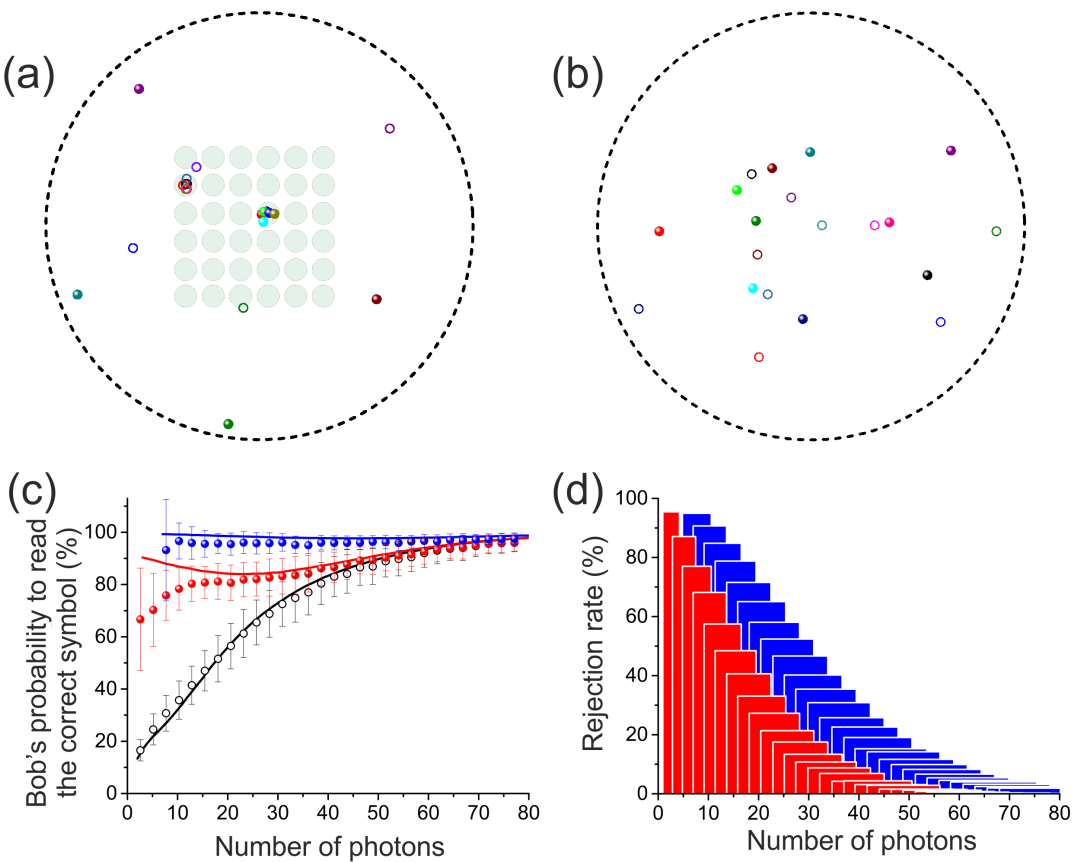

Figure 6.2 Experimental demonstration of secure communication. (a), (b). Snapshots of the spatial distributions of photons measured by Bob (a) and by Eve (b) in case of a perfect single-photon source and a fidelity of wavefront shaping $\alpha^{2}=0.6$. The open and filled dots correspond to two different symbols sent by Alice. Different colors represent 10 different repetitions of each of the symbols. The dashed line indicates the fiber core edge. Faint green circles on Bob's facet represent the symbol areas. In contrast to the photon distribution measured by Eve, Bob clearly sees the correlation between different realizations of the same symbol. (c). The measured probability of Bob to detect the correct symbol versus the average number of photons in a pulse for $\alpha^{2}=0.1$, weak coherent light source and different strategies: Bob uses no threshold (black); Bob accepts only symbols that have been triggered by two or more photons (red) and three or more photons (blue). Vertical error bars represent the standard deviation after averaging over 36 symbols. Solid lines show the theoretical calculations of the probabilities as described in the main text. (d). The rejection ratio for a threshold of 2 photons (red bars) and 3 photons (blue bars) versus the average number of detected photons in a pulse. The width of the bars corresponds to the standard deviations of the number of photons in a pulse and increases according to a Poisson distribution. 
old of 2 photons and 3 photons, respectively. Although even in the case of low fidelity of wavefront shaping $\left(\alpha^{2}=0.1\right)$ and no threshold, the probability of reading the correct answer is significant, it dramatically increases for strategies with a threshold. The downside is that Bob needs to reject all symbols below the threshold. Good agreement between experimental results and calculations allows us to use the theory to predict probabilities for different parameters of wavefront shaping. The rejection rates are also carefully analyzed and presented in Fig. 6.2 (d) for a threshold of 2 photons (red bars) and 3 photons (blue bars). The width of the bars corresponds to the standard deviation of the number of photons in a pulse and increases according to the Poisson distribution. As a result, Bob can read information with an error rate close to zero.

\subsubsection{Security with a single-photon light source}

Let us assume that the eavesdropper can tap off the signal somewhere in the middle of the fiber. In contrast to Bob, Eve does not have preliminary information about which field distributions correspond to what symbol. She needs to analyze the complicated field pattern created by the interference of numerous fiber-guided modes. Moreover, low-photon realizations provide insufficient information about the field structure, making it impossible to reconstruct the full propagating field. Single-photon measurements in a wrong basis provide information neither about the correct symbol nor about the correct basis. There are numerous possible bases and Alice and Bob do not need to switch basis. Remarkably, the method can remain secure even if we assume that Eve knows the basis, if we continue to assume that Eve cannot build a passive optical device that projects the $S$ wavefronts to $S$ single-photon detectors. In this scenario, where Eve is somehow able to fully characterize the fibers between Alice and Eve and Eve and Bob before the start of the transmission, she can only retrieve a fraction of the information that was sent by taking snapshots of the complex wavefronts in the fiber. In the Methods section, we derive a strict upper limit for the amount of information that Eve can gain in that case as $\left\langle H_{E}\right\rangle=(1-\gamma) / \ln 2 \approx 0.61$ bit per transmitted symbol regardless of the number of symbols or the number of modes, where $\gamma$ is the Euler constant. Under the same conditions, Bob at maximum can have $H_{B}(S)=\log _{2}(S) \approx 5.2$ bit of information per transmitted symbol for $S=36$ symbols. As a result, $\left\langle H_{E}\right\rangle \ll H_{B}$, guaranteeing the security of the method.

\subsubsection{Security with a weak coherent light source}

If rather than single photons, weak coherent light is used, phase measurements are possible, in principle. The fundamental limit of the best possible fidelity $\beta$ with which Eve can measure the wavefront with a low photon budget is determined by the following expression: $\beta=\left\{\bar{n}_{a} /\left(\bar{n}_{a}+2 N\right)\right\}^{0.5}$, where $\bar{n}_{a}$ is the 
average number of photons per pulse sent by Alice and $N$ the number of fiberguided modes (see the section 6.3). When Eve attempts to measure the field, estimates the symbol and re-sends the field to Bob, the intensity in the correct position on Bob's side will scale by $\beta^{2}$, according to the definition of the fidelity [160]. This intervention will, hence, be easily detected by Bob. We calculate Bob's probability to read the correct symbol after Eve's interruption by using the theory described in the previous section. The results are presented in Fig. 6.3(a), where solid lines show the probability without Eve's measurements and the dashed lines show the probability after Eve intercepts all photons and re-sends the measured field. Different colors correspond to different wavefront shaping efficiencies: blue, $\alpha^{2}=0.1$; green, $\alpha^{2}=0.3$ and red, $\alpha^{2}=0.5$. We see a huge drop in probability to detect the correct symbol in case of Eve's measurements, which can be clearly seen by Bob. This strategy hence won't allow Eve to intercept the complete signal without being detected immediately.
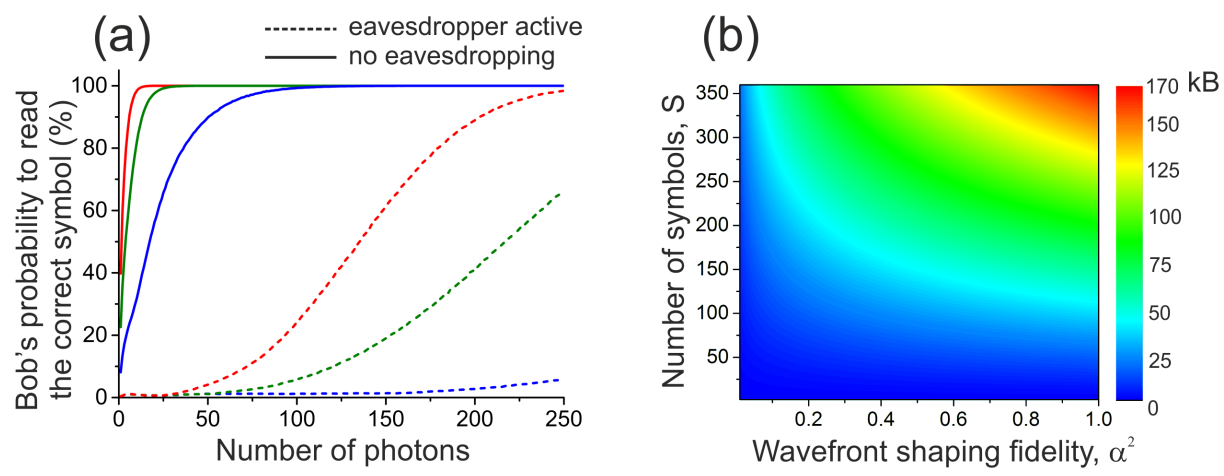

Figure 6.3 Security analysis for weak coherent light source if Eve does not know the optical transmission matrix of the fiber. (a). Bob's probability to read the correct symbol without Eve's intervention (solid lines) and after Eve intercepts all photons and re-sends the measured field (dashed lines) for different efficiency of wavefront shaping $\alpha^{2}=0.1$ (blue lines); $\alpha^{2}=0.3$ (green lines) and $\alpha^{2}=0.6$ (red lines). We see that this strategy does not allow Eve to intercept the complete signal without being detected immediately. (b). Length of the message (in kilobyte) that can be securely transferred from Alice to Bob before Eve can collect enough information, theoretically, to reconstruct the basis depending on the total number of symbols and the fidelity of wavefront shaping in case $\bar{n}_{a}$ of weak coherent light source is much less than the number of modes.

Eve can adopt another strategy in which she intercepts so little that it will be hard to detect. We assume that for low (relative to $\mathrm{N}$ ) photon number, as implied by $\beta^{2} \ll 1$, a deviation $\Delta n$ of more than one photon can be detected by Bob. In that case, we calculate (see section 6.3) the amount of information in bits 
I that can be safely transferred through the quantum channel with weak coherent light as a function of the quality of wavefront shaping $\left(\alpha^{2}\right)$ and the number of symbol used (S): $I=\left(1+2 \alpha^{2} N\right)^{0.5} S \log _{2} S$. The results are present in Fig. 6.3 (b). By increasing the fidelity of wavefront shaping and the number of symbols, we significantly increase the information which can be safely transferred in one single basis. It is important to highlight that we assumed the ideal case of Eve having infinite computational power to correctly distinguish all symbols. In practice, this is a complicated cluster-analysis task that may require significantly more information to reconstruct the basis correctly.

As long as Eve does not know the basis, the communication scheme for a low photon number $\left(\beta^{2} \ll 1\right)$ remains secure. It is amazing that - under some conditions - the method remains secure even if Eve gained the knowledge of the full field transmission matrix. Again, we assume that Eve cannot build a passive optical device that projects the $S$ different wavefronts onto $S$ detectors. We calculate the amount of information per transmitted symbol that Eve can retrieve at best for $N=1500$ fiber modes and $S=36$ symbols. The results are present in Fig. 6.4 (a) where the blue line represents the exact upper limit (evaluated through a numerical integration method) and the red line represents a simple closed-form analytical upper limit (see the Methods section for the details). We see that $H_{E}$ is below the maximum entropy achievable by Bob $\left(\log _{2} S\right)$ even when the average number of photons exceeds one. We analyzed the parameters (see the Methods section for the details) for which the amount of information per photon gained by Bob in imperfect conditions becomes more than the theoretically possible maximum of information gained by Eve even if she knows the basis (the green side in Fig. 6.4(b)). We see that even in the case where Eve has all possible information about the transmission channels and about the choice of symbols, our method is still secure provided that the number of symbols and wavefront shaping fidelity are high enough.

\subsection{Methods}

\subsubsection{Experimental setup}

Alice uses the continuous-wave linearly polarized output of a He-Ne laser with a wavelength of $633 \mathrm{~nm}$ (see Fig. 6.1 'Alice'). An attenuator based on neutral density filters in combination with a half-wave plate and a polarized beam splitter is used to reduce the power to the desired level. A single-mode fiber is used to clean the laser mode and to expand the laser beam in order to match the surface of a spatial light modulator. To control the spatial phase of the coupled light with high speed, Alice uses a $1920 \times 1200$ Vialux V4100 digital micromirror device (DMD). The lenses L1 and L2 are placed in a 4f-configuration to image the phase mask on the back focal plane of a coupling objective. A pinhole in 

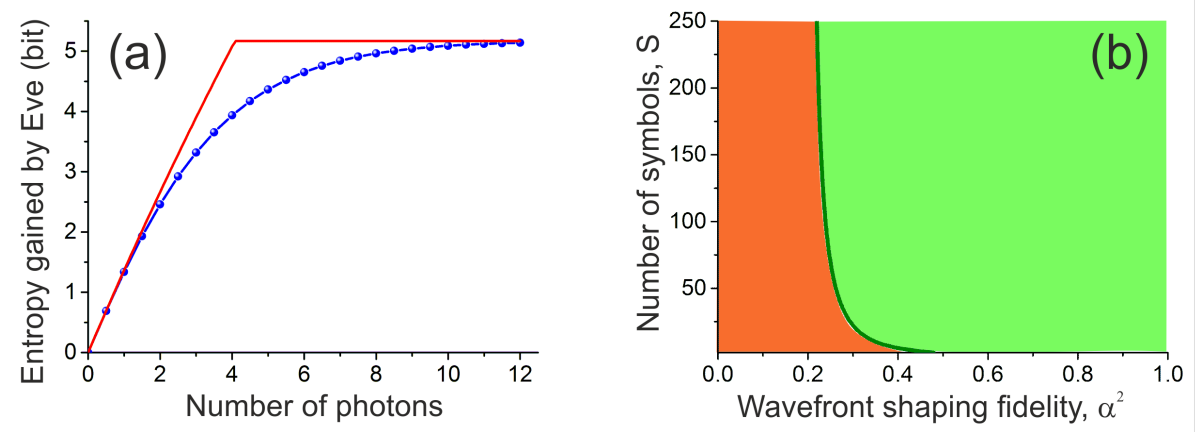

Figure 6.4 Security analysis for weak coherent light source in the scenario where Eve is assumed to know the exact optical transmission matrix of the fiber. (a). Entropy per transmitted symbol gained by Eve for $N=1500$ fiber modes and $S=36$ symbols. The connected blue dots represent the exact entropy function (evaluated through a numerical integration method) and the red line is a closed form expression that gives a simple upper limit (see Methods section). The result is independent of the wavefront shaping fidelity. (b). Parameter space (wavefront shaping fidelity and number of symbols) for which the amount of information gained by Bob per single photon under imperfect conditions is higher (green side) or lower (red side) than the theoretically possible maximum of information gained by Eve, assuming she knows the used basis. 
the Fourier plane blocks all the diffraction orders except the 1st, which encodes the desired spatial phase distribution. An objective with $N A=0.4$ (Olympus) is used to couple light into a conventional step-index multimode fiber (Thorlabs, FG050UGA) with a silica core of $d_{c}=50 \mu \mathrm{m}$ diameter and $N A=0.22$. Such a fiber has a normalized frequency $V=\pi d_{c} N A / \lambda \approx 55$ for our laser wavelength, $\lambda=633 \mathrm{~nm}$, and sustains approximately $N=1500$ guided modes [148]. The part of the experimental setup described above belongs to Alice and allows encoding the desired symbol with a speed of up to $10 \mathrm{kHz}$. The multimode fiber serves as a quantum channel to deliver symbols to Bob in such a way that anyone who wants to listen to the communication at any arbitrary position along the fiber fails. We mimic the situation when Eve has access to the fiber without Alice and Bob knowing so, dividing the quantum channel into two parts. Each piece of the multimode fiber is 3 meters long. Eve's part of the setup includes two objectives (Olympus, $N A=0.4$ ) served to close a gap between two pieces of fiber and to image fiber output on her camera (see Fig. 6.1 'Eve'). Bob's part of the setup includes one objective (Olympus, $N A=0.4$ ) to image the fiber output on the camera (see Fig. 6.1 'Bob'). Bob and Eve use a high-sensitive ICCD camera to detect the single-photon events (see 'Image processing' section). As a result, light can follow two potential pathways: first without mirror M2 through the both fibers (Bob's pathway) and second through mirror M2 through only the first fiber (Eve's pathway). During the calibration and characterization of Bob's part we use the first pathway and during the characterization of Eve's part we use the second pathway.

\subsubsection{Calibration and wavefront shaping procedure}

The complex wavefront-shaping algorithm to create a focused laser spot on the fiber output facet without information about the configuration of the fiber is as follows. We use the DMD to control the spatial phase profile of light at the fiber output facet. Each mirror of the DMD can be set to two different tilt angles. By controlling the tilt of every mirror, 2D binary blazed gratings can be created. With an appropriately tilted input light field, the diffracted light propagates along the normal of the DMD surface (see Fig. 6.1 'Alice'). An unoptimized plane wave that is incident on the fiber input gives rise to a speckle pattern on the output fiber facet. The Lee amplitude holography method is used to create the focused spot at the output. The DMD area of $510 \times 510$ pixels covered by an expanded laser beam is divided into $34 \times 34$ segments. Each segment consists of $15 \times 15$ micromirrors. Alice modulates the phase of each segment by shifting the grating pattern over $2 \pi$ in three steps in a random order. In total 3468 different phase masks were used. The security of the wavefront-shaping procedure is based on the same arguments as that of the security the quantum communication after completion of the wavefront shaping: the no-cloning theorem forbids an attacker to fully characterize the light pulse containing fewer photons than the 
number of fiber modes and scrambled by the multimode fiber. To guarantee the security of the wavefront shaping procedure, each pulse Alice sends contains only $80 \pm 30$ photons. In our experiments, 50 randomly-ordered repetitions of each phase profile are used. Bob measures the intensity in $S=36$ points on the fiber output facet for each phase pattern and sends this information back to Alice via a classical channel. In our experiments, a grid of $6 \times 6$ points with $3.2 \mu \mathrm{m}$ step size on the fiber output is used. However, the position of the symbols is not restricted and can be selected randomly. Alice recalculates the phase patterns leading to the highest intensity in each of 36 spots on the fiber output. The time required for the optimization procedure is 3 minutes and limited by the frame rate of the camera we used. We have characterized the fidelity (efficiency) of the wavefront shaping by the parameter $\alpha^{2}=P_{f} / P_{0}$, where $P_{f}$ is the power in the focus area with a center corresponding to that of the focal spot and a diameter equal to the FWHM of the Gaussian spot. $P_{0}$ is the total power on the fiber output, which is not changed by wavefront shaping. In the presented experiments, $\alpha^{2}$ is $10 \%$. In our experimental setup, the fidelity was limited by Eve's interception part in the transmission line (see Figure 6.1). In practice, an uninterrupted fiber should be used. For such fibers, a fidelity of $\pi / 4$ was reported experimentally for phase-only wavefront shaping [152].

\subsubsection{Image processing}

The signal was recorded by a HiCAM 5000 High-speed Intensified Camera (Lambert Instruments, the Netherlands) with $512 \times 512$ pixels and a speed of 5000 fps. To keep well-defined sensitivity, the incident photon flux is restricted to not exceed 5 detection events per frame on average. To investigate the parameters of secure communication with a higher number of photons, we summed up the required number of frames measured in the same conditions. As a result, an event with two or more photons at one point can be easily detected. The noise level was $3.3 \%$.

\subsubsection{Information gained by Bob}

We assume that Bob has $S$ detectors, where $S<N$. The maximum amount of information that Bob can read out per received photon is $H_{B}=\log _{2}(S)$. In case of $S=36$ symbols this maximum value is $H_{B} \approx 5.2$ bit of information. However, for a real-life situation, the fidelity of wavefront shaping will not be unity. The information Bob can get is in this scenario can be calculated as

$$
H_{B}=H(B)-H(B \mid S),
$$

where $H(B)$ is the entropy of the received alphabet, $H(B \mid S)$ the conditional entropy at the receiver side under the condition alphabet $S$ was sent. In further 
calculations we consider the single photon case. The probability of getting a click on detector $b$ in a case a random unknown symbol is sent is

$$
P(b)=\frac{\alpha^{2}}{S}+\frac{1-\alpha^{2}}{N}
$$

where $N$ is the number of modes, $S$ is the number of symbols, and $\alpha^{2}$ is the fidelity of the wavefront shaping. The total entropy, given that there was a click on one of the detectors is

$$
H(B)=-\sum_{b=1}^{S} P(b) \log _{2} P(b)=-S\left(\frac{\alpha^{2}}{S}+\frac{1-\alpha^{2}}{N}\right) \log _{2}\left(\frac{\alpha^{2}}{S}+\frac{1-\alpha^{2}}{N}\right) .
$$

Now we calculate the conditional entropy $H(B \mid S)$, which is the amount of information that is needed to describe measurement outcome $B$ given that the symbol $S=s$ is known. The probability of getting a click on detector $b$, given that symbol s was sent is

$$
P(b \mid S=s)=\alpha^{2} \delta_{b, s}+\frac{1-\alpha^{2}}{N} .
$$

As a result, the conditional entropy is

$$
H(B \mid S)=-(S-1)\left(\frac{1-\alpha^{2}}{N}\right) \log _{2}\left(\frac{1-\alpha^{2}}{N}\right)-\left(\alpha^{2} \frac{1-\alpha^{2}}{N}\right) \log _{2}\left(\alpha^{2} \frac{1-\alpha^{2}}{N}\right) .
$$

Using equations (6.1), (6.3) and (6.5) we find the information Bob can get per single photon detection in case of non-perfect wavefront shaping is

$$
\begin{aligned}
H_{B} & =-S\left(\frac{\alpha^{2}}{S}+\frac{1-\alpha^{2}}{N}\right) \log _{2}\left(\frac{\alpha^{2}}{S}+\frac{1-\alpha^{2}}{N}\right)+ \\
& +(S-1)\left(\frac{1-\alpha^{2}}{N}\right) \log _{2}\left(\frac{1-\alpha^{2}}{N}\right)+\left(\alpha^{2} \frac{1-\alpha^{2}}{N}\right) \log _{2}\left(\alpha^{2} \frac{1-\alpha^{2}}{N}\right) .
\end{aligned}
$$

This value was used to analyse the parameters for which the amount of information per photon gained by Bob in imperfect conditions becomes more than the theoretically possible maximum of information gained by Eve.

\subsubsection{Attacker model}

We aim at proving security of our multimode-fiber-based method of communication. In particular, we give security bounds in the case where Eve's attack on the quantum channel is not restricted. We consider the following classification of attacker models, according to the security review by Scarani et al. [26] 
We analyze individual attacks and their essential subfamily, the intercept-resend attacks. As the name indicates, in this class of attacks, Eve intercepts the signal somewhere on its way from Alice to Bob, performs a measurement on it, and prepares a new signal that she sends to Bob (see Fig. 6.1). The part of the fiber controlled by Bob is inaccessible to Eve.

Collective attacks, which imply that Eve keeps data in a quantum memory until the end of the classical post processing, are, fortunately, not applicable for our method. The great advantage of the multimode-fiber-based method is that it doesn't require a quantum key sifting step or any other classical post processing. General attacks, includes many possible variations and cannot be efficiently parametrized. Nevertheless, bounds for unconditional security have been found in many cases and in all these cases, it turns out that the bound is the same as for collective attacks [26].

Hacking attacks are related to the weaknesses of a practical implementation. The best-known example is the family of Trojan horse attacks, in which Eve probes the settings of Alice's and/or Bob's devices by sending some light into the system and register the reflected signal. However, in the setup where light goes only one way, the solution against Trojan horse attacks consists in using an optical isolator [26], which can be easily implemented in our setup.

We identify a new family of attacks based on the specific nature of our method: Making a functional physical copy of the MMF fiber, including the part controlled by Bob or -equivalently- a passive optical device that emulates the fiber. This requires precise replication of the combined effect of all bendings, inhomogeneities, temperature profile, etc. of a long multimode fiber. We assume that this is not possible with current technologies. To enhance the situation, the multimode fiber can be easily combined with a proven Physical Unclonable Function based on multiple-scattering media [161-163]. In the following security analysis the losses of the fiber are not considered. In the presence of loss an eavesdropper could exploit them by wiretapping the fiber immediately after leaving Alice's control. We expect that this case can be taken into account in a more refined analysis.

\subsubsection{Security analysis for a single-photon Fock state}

We assume that Alice uses a perfect single-photon source to send symbols and Eve can read the signal somewhere in the middle of the fiber, potentially close to Alice or close to Bob. We further assume that Eve cannot reproduce a real multimode fiber or build an ideal mode sorter (i.e., an optical system that completely reproduces the fiber between Eve and Bob without introducing significant losses). Additionally, since Alice sends a single photon, we assume that Eve performs intensity measurements. We now consider the most pessimistic scenario where Eve knows the basis: what field arrives at pixel $e$ of her detector when Alice sends a given symbol s. Let's call this the field transmission function 
$t_{e s}$. Neglecting losses and assuming Eve has a perfect detector (with $N$ pixels, where $N$ is the number of modes in the fiber), she will get exactly one click on one pixel of her detector for each symbol that is sent. We are now interested in calculating how much information Eve obtains by recording this single photon. The maximum amount of information that Eve can get is

$$
H_{B} \equiv H(E)-H(E \mid S)
$$

Here, the entropy $H(E)$ is the amount of information that is needed to describe a measurement outcome $\mathrm{E}$ and the conditional entropy $H(E \mid S)$ is the amount of information that is needed to describe this outcome when it is known which symbol $S$ was sent. By Bayes' rule for conditional entropy, the difference between the two entropies $H_{E}$ is the maximum amount of information that Eve can possibly gain. We are now left with the task of calculating the conditional entropy given that the symbol $S=s$ is known.

$$
H(E \mid S=s)=-\sum_{e}^{N} P(e \mid s) \log _{2} P(e \mid s),
$$

with $P(e \mid s) \equiv\left|t_{e s}\right|^{2}$ the probability for a photon sent as symbol s to arrive at pixel $e$. Since there are no losses, $\sum_{e}^{N}\left|t_{e s}\right|^{2}=1$. The exact value of $H(E \mid S=s)$ depends on the unknown, random transmission matrix elements and, therefore, is impossible to predict in advance. However, we can readily find the ensemble averaged value $\langle H(E \mid S=s)\rangle$, i.e., the conditional entropy averaged over all possible random transmission matrices of the fiber. To do so, we assume that $\left\langle\left|t_{e s}\right|^{2}\right\rangle=1 / N$ and that the elements $\left|t_{e s}\right|^{2}$ are drawn from independent exponential distributions. In the case of a large number of modes $N$, the distribution of $\left|t_{e s}\right|^{2}$ equals $P\left(\left|t_{e s}\right|^{2}\right)=N \exp \left(-\left|t_{e s}\right|^{2} N\right)$. To calculate the expected value for $H(E \mid S=s)$, we average over realizations of disorder. Substituting $y_{e s} \equiv\left|t_{e s}\right|^{2} \mathrm{~N}$ we can write

$$
\langle H(E \mid S=s)\rangle \equiv-\frac{1}{N} \sum_{e}^{N} \int_{0}^{\infty} \exp \left(-y_{e s}\right) y_{e s} \log _{2}\left(\frac{y_{e s}}{N}\right) d y_{e s} .
$$

Since the distribution of $y$ does not depend on $s$ or $e$, we can omit averaging over all $N$ symbols

$$
\langle H(E \mid S)\rangle=-\int_{0}^{\infty} \exp (-y) y\left(\log _{2} y-\log _{2} N\right) d y=\log _{2} N-\int_{0}^{\infty} \exp (-y) y \log _{2} y d y .
$$

This integral evaluates to

$$
\langle H(E \mid S)\rangle=\log _{2} N-\frac{1-\gamma}{\ln 2},
$$


with the Euler constant $\gamma \approx 0.577216$. Using equation (6.1) we finally find

$$
\left\langle H_{E}\right\rangle=\frac{1-\gamma}{\ln 2} \approx 0.61 \text { bit. }
$$

Hence, Eve gains only 0.61 bit of information per transmitted symbol at best, regardless of the number of symbols or modes even in the extreme case when she knows the exact basis. Under the same conditions, Bob will have at maximum $H_{B}(S)=\log _{2}(S) \approx 5.2$ bit of information per transmitted symbol for 36 symbols. As a result, $\left\langle H_{E}\right\rangle \ll H_{B}$, guaranteeing the security of the method.

\subsubsection{Security analysis for coherent state - intensity measure- ment}

From the derivation above it directly follows that Eve can gain a maximum of 0.61 bit of information if she records a single photon, regardless if this photon originated from a Fock state or a coherent state. Consequently, when Eve performs an intensity measurement on a coherent state, she can gain a maximum of 0.61 bit of information per recorded photon. Since there will be some degree of mutual information between these photons, the 0.61 bit per photon is a strict upper limit.

\subsubsection{Security analysis for coherent state - field measurement, unknown basis}

In the case that Alice sends a low-intensity coherent state, Eve may be able to set up a local oscillator reference and perform an interferometric measurement to measure the optical field at the interception point. Here we assume that Eve doesn't know the basis (see 'Calibration and wavefront shaping procedure' section.). The fundamental limit of the best possible fidelity $\beta$ with which Eve can measure the wavefront with low photon budget in case of weak coherent light source is determined by the following expression [160]: $\beta=\left\{\bar{n}_{a} /\left(\bar{n}_{a}+2 N\right)\right\}^{0.5}$, where $\bar{n}_{a}$ is the average number of photons per pulse sent by Alice and $N$ the number of fiber-guided modes. The factor 2 appears due to the complicated structure of the fiber-guided modes that mostly include two orthogonal polarizations and consequently requiring two measurements to be done. When Eve attempts to measure the field, estimates the symbol and re-sends the field to Bob, the intensity in the correct position on Bob's side will scale by $\beta^{2}$, according to the definition of the fidelity [160]. This intervention will, hence, be easily detected by Bob, as described in the main text.

Eve can adopt another strategy in which she intercepts so little that it will be hard to detect. The difference $\Delta n$ in the correct symbol photon number on Bob's side after Eve tapped off and re-sent $\bar{n}_{E}$ photons is $\Delta n=\alpha^{2} \bar{n}_{E}\left(1-\beta^{2}\right)$. We assume that for low photon number $\beta^{2} \ll 1$ and that a deviation $\Delta n$ of more 
than one photon can be detected by Bob. Consequently, to be undetected, Eve should use on average less than $1 / \alpha^{2}$ photons from each pulse. The fraction of the information that Eve receives from such a measurement is equal to the fidelity $\beta=\left(1+2 \alpha^{2} N\right)^{-0.5}$. The message length that Eve should accumulate before she can theoretically collect the full information about the current basis is $L=S / \beta$. As long as Eve does not know the basis, the communication scheme stays secure.

\subsubsection{Security analysis for coherent state - field measurement, known basis}

In the following, we again assume that Eve has somehow gained the knowledge of the full field transmission matrix $t_{e s}$ (which is an extremely pessimistic and unlikely scenario) to find a strict upper limit to the amount of information that Eve can retrieve. We assume that Eve is somehow able to record the field in each of the fiber modes independently, e.g., by a homodyning technique. The accuracy of these measurements will be limited by shot noise in the reference. When Alice sends a symbol $s$ with field amplitude $\mu$, at Eve's side the field in mode e equals $E_{e}=\mu t_{e s}$. However, due to shot noise, Eve will actually measure $\left(\boldsymbol{E}_{E v e}\right)_{e}=\mu t_{e s}+\xi_{e}$, with $\xi_{e}$ a noise term with $\bar{\zeta}_{e}=0$ and $\overline{\left|\xi_{e}\right|^{2}}=1$ photon, where $\div$ indicates averaging over measurements [160].

We now proceed to calculate the amount of information that Eve gains by doing this measurement. Eve can first project her measurement of the field at the fiber output $\boldsymbol{E}_{E v e}$ on the basis of symbols by simply multiplying with $t_{e s}^{-1}$. When all modes of the fiber are used $(S=N)$ and the fiber is lossless, $t_{e s}$ is unitary. After performing the transform, Eve will have found the vector $\boldsymbol{E} \equiv t_{e s}^{\dagger} \boldsymbol{E}_{E v e}$, where each element in $\boldsymbol{E}$ corresponds to a symbol. Averaged over measurements we find $\overline{\boldsymbol{E}}=\mu_{s}$, where $\mu_{s}$ is a vector a value of $\mu$ at index $s$, and zero everywhere else. Importantly, since the transform is unitary and the noise in different components of $\boldsymbol{E}_{E v e}$ is uncorrelated, after transformation the noise on each element of $\boldsymbol{E}$ will have the same statistics as before: i.e., the noise term has a complex Gaussian distribution with $\overline{\left|\xi_{e}\right|^{2}}=1$ photon. When $S<N$, it is still possible to define a unitary matrix $t_{e s}$ that maps all possible symbols to the first $S$ indices of $E$ and maps all unused state to the remaining indexes. Eve can simply discard the values at these remaining indices since they will never contain any information. Therefore, below we consider $\boldsymbol{E}$ to be a vector of length $S$. When Alice sends symbol $S=s$, the transformed vector $\boldsymbol{E}$ is drawn from the probability density function

$$
P(\boldsymbol{E} \mid S=s)=\frac{e^{-|| \boldsymbol{E}-\mu_{S} \|^{2}}}{\pi^{S}} .
$$

The differential entropy of this complex multivariate normal distribution is given by $H(E \mid S=s)=S \log _{2} \pi+S / \ln (2)$. We need to compare this value to the 
entropy for the case that it is not known what symbol was sent. In this case, the probability density equals

$$
P(\boldsymbol{E})=\frac{1}{S} \sum_{S=1}^{S} \frac{e^{-\| \boldsymbol{E}-\left.\mu_{S}\right|^{2}}}{\pi^{S}} .
$$

Unfortunately, there is no known closed form expression for the entropy of such a Gaussian mixture [163]. Therefore, we use a Monte Carlo approach to calculate the entropy of equation (6.8) numerically (see Figure 6.3(c)). Additionally, it is straightforward to derive an upper limit for the entropy. First, we realize that the real parts of the components of $\boldsymbol{E}$ all have a mean value of $\mu /$ Sand a variance of

$$
\frac{1}{2}+\frac{S-1}{S}\left(\frac{\mu}{S}\right)^{2}+\frac{1}{S}\left(\mu-\frac{\mu}{S}\right)^{2}=\frac{1}{2}+\frac{\mu^{2}(S-1)}{S^{2}}
$$

The imaginary parts have a mean of 0 and a variance of $1 / 2$. The maximum entropy distribution for a given variance is Gaussian. Therefore, our distribution (see equation (6.14)) must have a lower entropy than a Gaussian with the same variance. The differential entropy for this Gaussian is simply given by

$$
H(\boldsymbol{E})_{\text {upper }}=\frac{1}{2} \log _{2}(|2 \pi e \Sigma|)=S \log _{2} \pi+\frac{S}{\ln (2)}+\frac{S}{2} \log _{2}\left(\left(1+2 \mu^{2} \frac{(S-1)}{S^{2}}\right)\right.
$$

with $\Sigma$ the covariance matrix, which is diagonal in our case. Half of the diagonal elements are $1 / 2+\mu^{2}(S-1) / S^{2}$ (for the real parts), the rest (for the imaginary parts) is $1 / 2$, so $|2 \pi e \Sigma|=(\pi e)^{S}\left(\pi e\left(1+2 \mu^{2}(S-1) S^{-2}\right)\right)^{S}$. The upper limit for the entropy gained by Eve follows by subtracting $H(E \mid S=s)$, and also realizing that Eve cannot collect more information than what is being sent by Alice.

$$
H_{E}<\min \left[\frac{S}{2} \log _{2}\left(1+2 \mu^{2} \frac{S-1}{S^{2}}\right), \log _{2} S\right] .
$$

As long as, $H_{E}<H_{B}$ the method remains secure even in the extreme case of a weak coherent light source with more than 1 photon per pulse in a non-secret basis.

\subsection{Conclusion}

We demonstrated a new type of quantum communication method based on encoding secure information into photons decomposed over guided modes of a multimode fiber by using wavefront shaping. Unique properties of the method we introduced here allow data to be transferred in a deterministic manner and 
remove the necessity of a quantum key sifting step while simultaneously preventing Eve to access the secure information; Bob can decode the information instantaneously during the communication. As we have shown in the previous section, the method guarantees the security of the transferred information if a single-photon source is used. As a result, we can use the quantum channel for direct communication, realizing a completely new QSDC scheme.

We demonstrated that even for weak coherent light with a low (relative to the number of modes) photon number, our quantum communication scheme remains secure if the length of the message transmitted with the same fiber configuration doesn't allow Eve to gain the knowledge of the full field transmission matrix. We have shown that even in the extreme case when the exact optical transmission matrix of the fiber is known to Eve, the method remains secure if the average number of photons is low enough.

In our experiments, the bit rate was limited by the speed of the camera. With very fast cameras or APD arrays, the limitation of the proposed method might be the speed at which phase masks can be changed. Digital micromirror device together with the high-dimensionality of the used alphabet $\left(\log _{2} 36=\right.$ 5.2 bit per pulse) gives rise to a secure bitrate up to $118 \mathrm{kbps}$. The bit rate can be increased twofold without any changes in the setup and equipment by increasing the number of symbols [134]. Further improvements can be made by increasing the number of guided modes of the multimode fiber. Commercially available multimode fibers support up to $3 \cdot 10^{6}$ modes, which is 2000 times more than we used in the present experiments. Such a fiber correspondently allows to increase the dimensionality of the quantum channel and consequently the level of security.

To summarize, we propose and implement a new quantum communication method that is based on high-dimensional spatial encoding through a multimode fiber and that can be used both for Quantum Key Distribution and for Quantum Secure Direct Communication. The method is secure with coherent light even when several photons per symbol are used. Moreover, the method requires only a simple practical setup, which can be naturally extended to long distances. 



\section{Comparison of Quantum Communication Methods}

In this chapter we review several quantum cryptography and quantum authentication protocols. We compare them by their security assumptions, their requirements on the quantum channel and the public channel, as well as on the dimensionality of their Hilbert spaces.

\subsection{Introduction}

Quantum information theory has enriched computer science with new cryptographic concepts, quantum algorithms that achieve spectacular speedups and with alternative interpretations of known phenomena. Relevant principles are the quantum no-cloning theorem [16] and quantum entanglement [20]. Relatively recent is the combination of Physical Unclonable Functions (PUFs) [62] as a security primitive with Quantum Readout $(\mathrm{QR})[164]$ to achieve what is called Quantum-Secure Authentication (QSA) [61, 165, 166]. Another concept, which seems completely unrelated to the principles mentioned above is Quantum Data Locking (QDL), put forward by DiVincenzo et al. [111]. QDL is the notion that in quantum information it is possible to have a bipartite quantum state which contains a large locked classical correlation which gets unlocked by a disproportionately small amount of classical communication. In this chapter, we will review Quantum-Secure Authentication and Quantum Data Locking and related quantum security protocols such as Quantum Key Distribution (QKD), in particular the BB84 protocol [13], Quantum-Secure Direct Communication (QSDC) [141], the recently proposed PUK-Enabled Asymmetric Communication (PEAC) [167] as well as Multimode Fiber Communication (MMFC) [137] and compare them from the information-theoretical perspective.

\subsection{Quantum-Secure Authentication}

Quantum-Secure Authentication is a method to authenticate optical PUFs by Quantum Readout. The method is illustrated in Fig. 7.1. The optical properties of the PUF are allowed to be publicly known. Fig. 7.1 represents an idealized case in which losses are neglected. The wavefront of a single-photon planewave light pulse is phase-modulated with a complex two-dimensional challenge pattern by a spatial light modulator (SLM1). The challenge is randomly chosen from a high-dimensional vector space. It is sent to the PUF, which is a multiplescattering sample with a unique microstructure which is unfeasible to copy. The

Parts of this chapter are based on: T. B. H. Tentrup, R. Uppu, B. Škorić, A. P. Mosk, and P. W. H. Pinkse, "Quantum-Secure Authentication vs Quantum Data Locking," in preparation. 
response wavefront is analysed by sending it to SLM2, which is pre-programmed with a phase pattern that is the conjugate of the expected response, resulting in a plane wave that is focused on the single-spatial-mode optical detector. The single photon will lead to a detector click signaling the authenticity of the PUF. The wrong response, e.g., caused by a fake PUF, will not focus down to the detector and no photodetection click will occur.

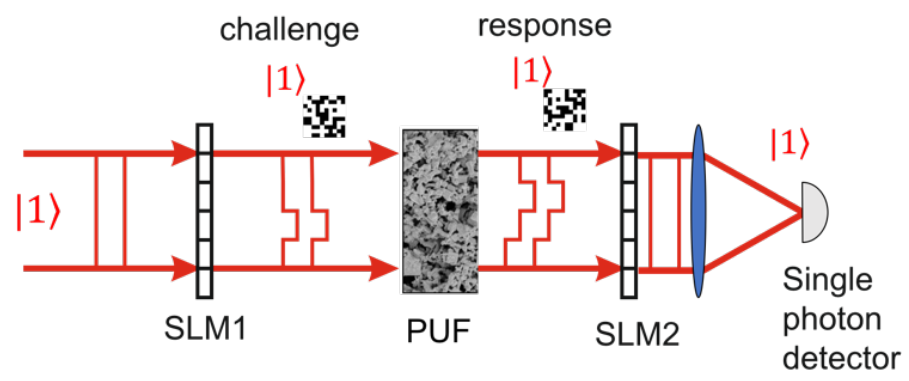

Figure 7.1 Schematic of Quantum-Secure Authentication [61] in the ideal case of a single photon and no losses. The challenge is formed by a plane-wave single-photon light pulse that is spatially shaped by SLM1, and sent to a Physical Unclonable Function (PUF) where it multiple scatters. The returned light is decoded by SLM2 and - in the case of the expected response - focused down on a single-mode single-photon detector.

The security of QSA depends on the technological infeasibility of cloning the PUF behavior. This "unclonability" is finding its way into optical security at an accelerating pace [168]. QSA is unconditionally secure against attempts to determine the challenge and then compute or look-up the corresponding response. The challenge space should be as large as possible and each challenge should be chosen randomly to minimize the chance of an attacker guessing the challenge. In a realistic scenario there are losses, but this can be compensated by using more light. It has been shown that QSA is secure if the number of photons in the challenge is smaller than the number of spatial channels in the wavefronts $[165,166]$. A real-life implementation should also contain detectors measuring the amount of light falling outside of the single-mode detector to prevent blinding attacks and should of course prevent an attacker from reading out the state of SLM1 and SLM2. QSA can also be used for the authentication of data in a protocol called QSA-d [169]. In this protocol, repeated verifications of the QSA type take place. In each cycle Bob, who wants to send a verified bit stream, presents one of two PUFs, representing logical " 0 " and " 1 ", which Alice then verifies. Bob may announce the bit stream beforehand over a classical channel, in which case Alice knows exactly which of Bob's PUFs to authenticate. On the other hand, QSA-d can also be implemented without such announcement; then Alice has to 
configure SLM2 such that two foci are created, one for each of Bob's PUFs. The security of QSA-d is equivalent to that of QSA. In the following we want to focus on the idealized situation. We analyse the information flow in QSA and other quantum security protocols concerning in particular the dimensionality of the used wavefronts and compare this to QDL.

\subsection{Quantum Data Locking}

The concept of quantum data locking was put forward in a paper by DiVincenzo et al. [111]. In their paper, they discuss the classical mutual information $I_{\mathcal{C}}\left(\rho_{A B}\right)$ of a quantum state $\rho_{A B}$, which is defined as the maximal classical mutual information $I(A: B) \equiv H\left(\rho_{A}\right)+H\left(\rho_{B}\right)-H\left(\rho_{A B}\right)$ that can be obtained by local measurements $M_{A} \otimes M_{B}$ on state $\rho_{A B}$. Here $H$ is the entropy function and $\rho_{A B}$, $\rho_{A}, \rho_{B}$ are the probability distributions of the joint and individual outcomes of performing the local measurement $M_{A} \otimes M_{B}$ on $\rho_{A B}$. Moreover, $I_{c}\left(\rho_{A B}\right)$ corresponds to the usual classical mutual information if $\rho_{A B}$ is completely classical. The classical mutual information has the following properties:

1. Monotonicity. Since $I_{\mathcal{C}}\left(\rho_{A B}\right)$ is a non-local property, it should not increase by local operations.

2. Total proportionality. Starting from an uncorrelated state and using $l$ qubits or $2 l$ bit of communication and local operations, one should not create more than $2 l$ bit of correlation.

3. Incremental proportionality. Transmission of $l$ qubits or $2 l$ bit should not increase the correlation of any initial state by more than $2 l$ bit.

The surprising discovery by DiVicenzo et al. was that (3) incremental proportionality can be violated for a quantum state. To illustrate QDL, DiVincenzo et al. considered the situation depicted in Fig. 7.2. Alice chooses $d=2^{K}$, picks a random $K$-bit string $|k\rangle$ and sends Bob $|k\rangle$ or $U_{C}|k\rangle$, where $U_{C}$ is a unitary transformation, depending on whether the random bit $t=0$ or 1 . Bob does not know in which basis to measure the state he received from Alice. Assuming the two possible basis choices are mutually unbiased, and assuming Bob randomly picks a basis, he will choose the correct basis with $50 \%$ probability and therefore, on average gains $K / 2$ bit of Shannon information. Of course, if he knows the right basis, he will gain $K$ bit of Shannon information. The single classical bit of information therefore unlocks $K / 2$ bit of information. The origin of this violation of incremental proportionality is connected to the quantum no-cloning theorem [16]: if the information sent by Alice would be classical, Bob can always make one or several copies of $|k\rangle$, measure the state in all possible bases and gain $K$ bit of information. Recently Lum et al. realized this idea experimentally 
in what they called the "Quantum enigma machine" [110] using a single-photon source and two SLMs.

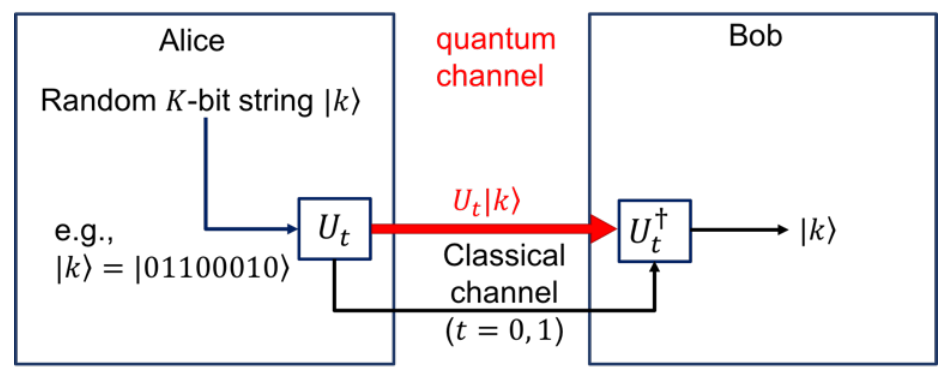

Figure 7.2 Quantum Data Locking. In the simplest example, exploited in the "Quantum Enigma" [110], the dimensionality of the classical information is 2, and $U_{0}=I$ and $U_{1}=H$. In this situation, a single bit of classical information unlocks $K / 2$ bit of information for Bob.

DiVincenzo et al. remarked that QDL is related to quantum key distribution (QKD), in particular, BB84 [13], in which Alice holds a basis bit for each of Bob's qubits. The transmission of the locked state limits the classical correlation between Alice and an eavesdropper (Eve) and forbids her from tampering without disturbance. The correlation is unlocked later by the classical communication between Alice and Bob revealing their basis choices. Eve is detected if test bits in the unlocked information are inconsistent. In other words, BB84 can be considered as a form of QDL in which one bit is sent for every bit to be unlocked, and there is no extreme unlocking behavior as shown by the example of Fig. 7.2. Equivalently, Quantum Key Recycling (QKR) which permits re-use of keys also possesses no extreme unlocking, as every key bit unlocks only one bit of information [170, 171]. Key-free schemes such as Quantum Secure Direct Communication (QSDC) usually rely on fragile quantum states (EPR pairs) where two bits of information are retrieved upon receiving both photons of the EPR pair by Bob [141]. However, loss of even one photon results in complete loss of information. QSDC schemes utilizing block coding of information over multiple single photons have also been proposed with $<1$ bit transmitted per 100 sent photons [144]. However, the security of the protocol is weaker than the conventional QSDC schemes. Therefore, we restrict ourselves here to EPR-based QSDC. In the following we compare the extreme data unlocking of QDL to QSA.

\subsection{QSA in terms of QDL}

The essence of QDL is incremental proportionality which can be violated in a quantum state. However, QDL also triggers a discussion on the dimensionality 
of quantum states and the information contained in them. As discussed above, DiVincenzo et al. looked at BB84 in this framework. Similar to QDL but in contrast to BB84, QSA uses a high-dimensional quantum state. In the following we analyse the analogy further.

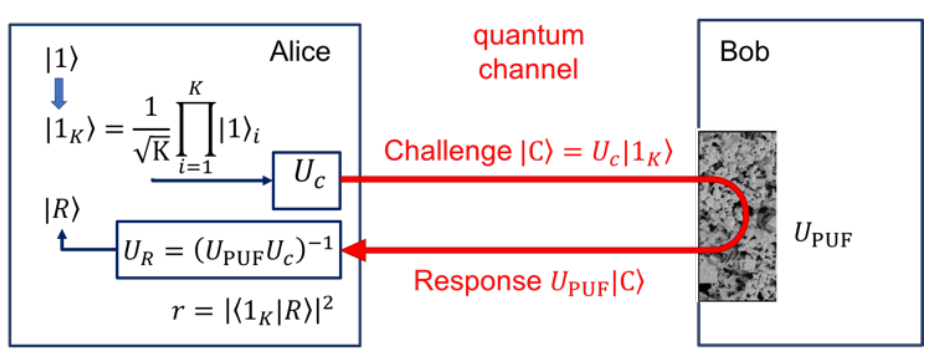

Figure 7.3 QSA in an abstract representation. The classical binary information, i.e., the question if Bob's key is authentic or not, is unlocked by the hidden information which transformation $U_{c}$ that Alice used to form the challenge wavefront.

Consider the idealized schematic representation of QSA in Fig. 7.3, where we ignore losses. Alice prepares a single photon in a high-dimensional state $|C\rangle=U_{C}\left|1_{K}\right\rangle$, where

$$
\left|1_{K}\right\rangle=\frac{1}{\sqrt{K}} \prod_{i=1}^{K}|1\rangle_{i}
$$

is the one-photon plain-wave input on the spatial modulator represented by $U_{C}$, which here is a randomly chosen unitary transformation. The resulting single-photon wavefront is sent to Bob's PUF where it multiply-scatters in a complex way that can be represented by the unitary transformation $U_{P U F}$. The response wavefront is transformed by Alice's second spatial light modulator represented by $U_{R}=\left(U_{P U F} U_{C}\right)^{-1}$. The overlap of the thus-transformed wavefront $|R\rangle$ with a single mode (corresponding to the plane wave focusing on the detector) is determined and constitutes the scalar signal $0<r<1$ that should be larger than a preset threshold for successful authenticity flagging.

In terms of QDL, one can say that in QSA the binary classical information ("is the physical key authentic") is unlocked by a $K$-dimensional quantum challengeresponse check, which will contain $\log _{2}(K)$ bit of information. Alice uses the randomly but secretly chosen $U_{C}$, which contains $K$ elements, as secret information in addition to the publicly known $U_{P U F}$, which is an $K \times K$ matrix. As discussed above, in contrast to the case with the most extreme unlocking behavior, BB84 has a 1:1 ratio of secret and unlocked bits, i.e., its unlock ratio is 1. In this sense QSA has an even lower unlocking ratio, as in QSA as a high- 
dimensional challenge-response is used to unlock only a single bit. Note that the "Quantum enigma machine" [110] can be used to build up a larger key from a pre-shared secret by subsequentially encoding the basis choice of the next photon in the previous photon. In contrast to QSA, where all information of the PUF is publicly known, in this protocol the unlocking classical bit must be kept secret.

An interesting question is what the information content of the few-photon challenge wavefront is. On the one hand, to describe it in detail, one needs to know the phases and amplitudes of a large number $\left(K\right.$, on the order of $\left.10^{3}\right)$ of spatial modes. On the other hand, it contains only a few photons. In the idealized case of a single photon, the information content will be given by $\log _{2}(K)$. It was recently demonstrated experimentally that with modern technology one can actually transfer more than 10 bit of information with a single photon [134].

\subsection{PEAC and MMFC}

PEAC is an asymmetric secure communication method using PUFs to add authentication of the two communicating parties to a BB84-type protocol. Alice sends few-photon wavefronts to focus light through a PUF onto one of two multimode fibers. The used fibers are chosen large enough to accept distinct foci originating from linearly independent incident wavefronts on their input facet. This makes it possible to have two subsets of wavefronts coupling to the two fibers. Hence, every superposition of a subset of these wavefronts will also couple to the fibers. The two multimode fibers are each connected to an avalanche photodiode representing one bit. The bit value is given by the detector with the larger count rate. The security of this communication method relies on the hardness to distinguish two subsets of few-photon complex wavefronts and the unclonability of PUFs. Only Bob can read the message with his PUF and no classical channel is needed.

MMFC is described in detail in chapter 6. A multimode fiber is used as the quantum channel between Alice and Bob. In multimode fibers the modes couple to each other, which results in a scrambling of input wavefronts and a speckle light image at the output facet. Wavefront shaping can undo this scrambling to encode information in the spatial domain by writing distinct foci. This wavefront shaping can be done with random wavefronts and only a few photons per wavefront to minimize the information a wiretapping eavesdropper could gain. Bob announces his photon number measurements at random positions of the output facet to allow Alice to generate the wavefronts to focus in these areas. After that, Alice and Bob share an alphabet with the focus positions on Bob's detector being the symbols. Now Alice can send the message with few-photon wavefronts. Since a wiretapping eavesdropper could sample the wavefronts over time, the transmission matrices need to be changed after a certain number of photons, 
which can be realized by randomly pertubating the multimode fiber at Bob's or Alice's end. The system needs to be characterized again by wavefront shaping. It can be seen as a form of QDL, where the transmission matrix of the fiber unlocks the information encoded in the wavefronts. Compared to PEAC, no PUF but a multimode fiber is employed. The fiber can be altered by Alice and BOB. Where in PEAC an attacker would have to distinguish two subsets of wavefronts, in MMFC the attacker needs to distinguish a set of wavefronts, which also changes before each wavefront shaping step.

\subsection{Wider Comparison}

\begin{tabular}{|c|c|c|c|c|c|c|c|}
\hline & $\begin{array}{c}1 \text { or } \\
2 \\
\text { way }\end{array}$ & $\begin{array}{l}\text { auth } \mathrm{q} \\
\text { channel }\end{array}$ & security & $\begin{array}{c}\text { data } \\
\text { transfer }\end{array}$ & $\begin{array}{l}\text { type } \\
\text { of key }\end{array}$ & $\begin{array}{l}\text { Hilbert } \\
\text { space di- } \\
\text { mention }\end{array}$ & $\begin{array}{c}\text { use of } \\
\text { classical channel }\end{array}$ \\
\hline QKD & 1 & no & uncond & yes & none & 2 & $\begin{array}{c}\text { auth; qubit selection; } \\
\text { noise testing }\end{array}$ \\
\hline QKR & 1 & no & uncond & yes & symm & 2 & masked message \\
\hline QDL & 1 & no & Not applicable & yes & symm & $>2^{2}$ & auth; unlocking \\
\hline QSDC & 2 & no & $\begin{array}{l}\text { Uncond } \\
\text { (no proof for } \\
\text { noisy channel) }\end{array}$ & yes & none & 2 or 4 & auth; noise testing \\
\hline QSA & 2 & yes & $\begin{array}{c}\text { PUF } \\
\text { unclonability }\end{array}$ & no & PUF & $K \approx 10^{3}$ & none \\
\hline QSA-d & 2 & yes & $\begin{array}{c}\text { PUF } \\
\text { unclonability }\end{array}$ & yes & PUF & $K \approx 10^{3}$ & full message \\
\hline PEAC & 1 & yes & $\begin{array}{c}\text { nonorthogonal } \\
\text { subspaces }\end{array}$ & yes & PUF & $K \approx 10^{3}$ & none \\
\hline MMFC & 1 & no & $\begin{array}{c}\text { indistinguishable } \\
\text { wavefronts }\end{array}$ & yes & $\begin{array}{c}\text { MMF } \\
\text { (non static) }\end{array}$ & $K \approx 10^{3}$ & auth; noise testing \\
\hline
\end{tabular}

Table 7.1 Comparison of Quantum Key Distribution (QKD), Quantum Key Recycling (QKR), Quantum Data Locking (QDL), Quantum Secure Direct Communication (QSDC), Quantum Secure Authentication (QSA), its data version (QSA-d), PUK-Enabled Asymmetric Communication (PEAC) and Multimode Fiber Communication (MMFC). We compare the directionality of the quantum channel in the protocol, whether the quantum channel is authenticated, the security, whether the protocol can be used for data transfer, the type of key, the dimensionality of the Hilbert space and finally the purpose/function of the classical channel. Note that in QSA, the PUF is the private key; the description of the PUF is the public key. For QKD we consider BB84, a 6-state or similar qubitbased protocol. QKD variants exist that have much less communication over the classical channel. On the other hand, also high-dimensional QKD variants exist $[48,49,52,55,173]$.

Let us end by drawing the comparison a little further. Table 7.1 lists the main properties of Quantum Key Distribution (QKD), Quantum Key Recycling (QKR), Quantum Data Locking (QDL), Quantum Secure Direct Communication (QSDC), 
Quantum Secure Authentication (QSA), its data version (QSA-d), PUK-Enabled Asymmetric Communication (PEAC) and Multimode Fiber Communication (MMFC). Concerning the directionality of the quantum channel, there is a clear distinction between QKD, QKR, QDL, PEAC and MMFC which all only use a one-way quantum channel, and QSA, QSA-d and those QSDC schemes that use an EPR pair which all use a two-way quantum channel. We remark that QKD, QKR, QDSC and MMFC require some way to authenticate the classical communication channel. Usually this is done by symmetric cryptography, i.e., an a priori shared secret is needed. On the other hand, QSA, QSA-d and PEAC provide an authenticated quantum channel and as such require no further means of authentication.

QSDC is the only protocol in the table that uses entanglement as a resource, a feature shared with some QKD schemes, in particular those employed for device-independent QKD [172]. The security of QKD, QKR and QSDC is unconditional, whereas QSA, (and QSA-d) is unconditionally secure against emulation attacks, but relies on PUF unclonability. Unclonability depends on an estimate of the attacker's technological capabilities and therefore cannot be proven rigorously. PEAC relies on the hardness to distinguish two nonorthogonal subsets of few-photon wavefronts, whereas it is the task to distinguish a periodically changing set of wavefronts which makes MMFC secure. For QDL as a theoretical concept to show incremental proportionality, security is not relevant. Since QSA is an authentication method, there is no data transfer, but in its offspring QSA-d there is. The table furthermore lists the dimensionality of the Hilbert space in the various schemes considered and the use of the classical channel.

\subsection{Conclusion}

We have compared several quantum-security protocols in terms of dimensionality of the Hilbert space, the security, type of key used, characteristic properties of the classical and quantum channels. In particular, we point out that in QSA a high-dimensional wavefront is used to securely unlock the information on the authenticity of the physical key. One should, however, be aware of important differences the various protocols: In QDL a receiver unlocks information using classical information obtained from a sender. In QSA there is a verifier (Alice) that authenticates a key in the possession of Bob. The large overhead in the dimensionality of the employed wavefronts to answer a binary question (is the PUF authentic?) here serves the purpose of making QSA secure against attacks without relying on any stored secrets. In this context PEAC and MMFC can be seen as a communication extension of the ideas of QSA. Finally, we also remark that QSA-d can be seen as a form of QSDC where the return channel is authenticated. 


\section{Summary and Outlook}

\subsection{Summary}

In this thesis, Quantum Key Distribution and quantum communication methods based on high-dimensional spatial coding of light are developed. Their security is based on the fundamental quantum nature of light and profits from the highdimensional Hilbert spaces offered intrinsically by imaging optics. An overview of classical information theory and quantum information is given to introduce the field of quantum cryptography. The advantages of larger dimensional alphabets and the security of the standard two-bases BB84 protocol is shown. The spatial states used for encoding are introduced. An analytic expression for the upper bound on the mutual information for these states is derived, including multiphoton states, detector noise and beam broadening. High-dimensional encoding of single photons is experimentally realized, reaching 10.5 bit per received photon. The dependence of the mutual information on the number of detector pixels is discussed and the experimental values are compared with the theoretical upper bound. It was shown that a standard error-correcting LDPC code is sufficient to achieve practically error-free communication. By adding a second mutually unbiased basis, a large-alphabet QKD system is experimentally realized and characterized. The security of this BB84-like protocol is analyzed in terms of intercept-resend and collective attacks. The key rate after postprocessing is analyzed under realistic circumstances, including finite key length. Finally, a new quantum communication method is demonstrated, which is based on encoding information into wavefronts decomposed over guided modes of a multimode fiber. At the end a step back is made and the similarities and differences of several quantum authentication and quantum cryptography schemes as well as Quantum Data Locking are discussed. This involves comparison of the characteristics of the classical channel, the quantum channel and the necessary dimension of the Hilbert space. 


\subsection{Outlook}

Finally, there are some recommendations and proposals for future experimental work on the subject of large-alphabet quantum communication. The first recommendation is on the technological optimizations which can be made. The bottleneck for the key generation rate in our BB84-based QKD experiment is the mechanical switching of the half-wave plates in the setup. This is needed to direct the light by a polarizing beam splitter to one of the two optical paths of the setup. This switching limits the basis switch rate to $1 \mathrm{~Hz}$ and could be done much faster with Pockels cells. The Pockels cell is an electro-optic device which allows switching speeds of $5 \mathrm{kHz}$. Another limiting factor is the speed of the liquid-crystal spatial light modulator. Its $60 \mathrm{~Hz}$ refresh rate can be increased up to $10 \mathrm{kHz}$ with modern Digital Micromirror Devices (DMDs). The main drawback of DMDs are higher losses of $\sim 95 \%$. Also a scanning galvo mirror can be considered, having a repetition rate of $\sim \mathrm{kHz}$, but no wavefront shaping capability. Finally, the key generation rate and communication rate for the Multimode Fiber Communication can be increased by using Superconducting Nanowire Single-Photon Detectors (SNSPDs). SNSPDs offer count rates of hundreds of $\mathrm{MHz}$ together with a quantum efficiency of $\sim 80 \%$ [174]. Unfortunately, they have a small active area due to the wiring and offer only around 100 pixels. Alternative detectors are Microwave Kinetic Inductance Detectors (MKIDs) [175]. This single-photon sensitive detector can be scaled to be used in large arrays.

A direct next step for the QKD experiment is to check the feasibility for large distance QKD. For line-of-sight free-space propagation the Spatial Light Modulator (SLM) can be used to investigate the influence of atmospheric turbulence on the channel capacity by using Kolmogorov turbulence phase screens [176]. The SLM could also be used to correct for turbulence and investigate the influence of this correction on the security of the QKD protocol. Another possibility is to use the SLM to encode information and change of basis by also considering a partially mutually unbiased basis.

For a practical realization of Multimode Fiber Communication (MMFC) the stability of the MMF over a few kilometer distance needs to be studied. That includes wavefront shaping and measuring the decorrelation time of the speckle pattern. Another step would be to add secure and multi-user authentication to MMFC by combining the MMF with a Physical Unclonable Function (PUF). 


\section{References}

[1] D. Kahn, The Codebreakers: The comprehensive history of secret communication from ancient times to the internet (Simon and Schuster, 1996).

[2] J. Trithemius, Polygraphiae libri sex (1518).

[3] A. Beutelspacher, Kryptologie (Vieweg, 1996)

[4] G. Vernam, "Secret signaling system," (1919). US Patent 1,310,719.

[5] C. E. Shannon, "Communication theory of secrecy systems," Bell Labs Tech. J. 28, 656-715 (1949).

[6] E. H. Hebern, "Electric coding machine," (1921). US Patent 1,510,441.

[7] A. Scherbius "Chiffrierapparat" (1918). DE Patent 416,219

[8] C. E. Shannon, "A mathematical theory of communication," Bell Syst. Tech. J. 27, 379-423 (1984).

[9] R. L. Rivest, A. Shamir, L. Adleman, "A method for obtaining digital signatures and public-key cryptosystems," Commun. ACM 21, 120-126 (1978).

[10] P. W. Shor, "Algorithms for quantum computation: Discrete logarithms and factoring," Proceedings 35th Annual Symposium on Foundations of Computer Science, Santa Fe, NM, 1994, pp. 124-134.

[11] M. Blum, "Coin flipping by telephone a protocol for solving impossible problems," ACM SIGACT News 15, 23-27 (1983).

[12] S. Wiesner, "Conjugate coding," Sigact News 15, 78-88 (1983).

[13] C. H. Bennett and G. Brassard, "Quantum cryptography: Public key distribution and coin tossing," in International Conference on Computers, Systems E Signal Processing, Bangalore, India, Dec 9-12, 1984, (1984), pp. 175-179.

[14] G. Brassard, "Brief history of quantum cryptography: A personal perspective," Proceedings of IEEE Information Theory Workshop on Theory and Practice in Information Theoretic Security, Awaji Island, Japan, October 2005 pp. 19 23.

[15] C. H. Bennett and G. Brassard, "Quantum cryptography and its application to provably secure key expansion, public-key distribution, and cointossing," Proceedings of IEEE International Symposium on Information Theory, St-Jovite, Canada, September 1983, pp. 91.

[16] W. K. Wootters and W. H. Zurek, "A single quantum cannot be cloned," Nature 299, 802-803 (1982).

[17] C. H. Bennett and G. Brassard, "The dawn of a new era for quantum cryptography: The experimental prototype is working," Sigact News 20, $78-82$ (1989).

[18] C. H. Bennett, F. Bessette, G. Brassard, L. Salvail, J. Smolin, “Experimental quantum cryptography," J. Cryptol. 5, 3-28 (1992). 
[19] A. K. Ekert, "Quantum cryptography based on Bell's theorem," Phys. Rev. Lett. 67, 661-663 (1991).

[20] A. Einstein, B. Podolsky, and N. Rosen, "Can quantum-mechanical description of physical reality be considered complete?," Phys. Rev. 47, 777780 (1935).

[21] J. S. Bell, “On the Einstein-Podolsky-Rosen paradox," Physics 1, 195-200 (1964).

[22] C. H. Bennett, G. Brassard, N. D. Mermin, "Quantum cryptography without Bell's theorem," Phys. Rev. Lett. 68, 557-559 (1992).

[23] C. H. Bennett, G. Brassard, and A. K. Ekert, "Quantum cryptography," Scientific American 267, 50-57 (1992).

[24] D. Mayers, A. Yao, "Quantum cryptography with imperfect apparatus," "Foundations of Computer Science, 1998. Proceedings.", 503-509 (1998).

[25] N. Gisin, G. Ribody, W. Tittel, and H. Zbinden, "Quantum cryptography," Rev. Mod. Phys. 74, 145-195 (2002).

[26] V. Scarani, H. Bechmann-Pasquinucci, N. J. Cerf, M. Dušek, N. Lütkenhaus, and M. Peev, "The security of practical quantum key distribution," Rev. Mod. Phys. 81, 1301-1350 (2009).

[27] M. A. Nielsen and I. L. Chuang, Quantum computation and quantum information (Cambridge University, 2010).

[28] H. Bechmann-Pasquinucci and N. Gisin, "Incoherent and coherent eavesdropping in the six-state protocol of quantum cryptography," Phys. Rev. A 59, 4238 (1999).

[29] G. Brassard, N. Lütkenhaus, T. Mor, B. C. Sanders, "Limitations on practical quantum cryptography," Phys. Rev. Lett. 85, 1330 (2000).

[30] N. Lütkenhaus, and M. Jahma, "Quantum key distribution with realistic states: photon-number statistics in the photon-number splitting attack," New J. Phys. 4, 44 (2002).

[31] V. Scarani, A. Acin, G. Ribordy, N. Gisin, “Quantum cryptography protocols robust against photon number splitting attacks for weak laser pulse implementations," Phys. Rev. Lett. 92, 057901 (2004).

[32] Y.-L. Tang, H.-L. Yin, S.-J. Chen, Y. Liu, W.-J. Zhang, X. Jiang, L. Zhang, J. Wang, L.-X. You, J.-Y. Guan, D.-X. Yang, Z. Wang, H. Liang, Z. Zhang, N. Zhou, X. Ma, T.-Y. Chen, Q. Zhang, and J.-W. Pan, "Measurement-deviceindependent quantum key distribution over 200 km," Phys. Rev. Lett. 113, 190501 (2014).

[33] H.-L. Yin, T.-Y. Chen, Z.-W. Yu, H. Liu, L.-X. You, Y.-H. Zhou, S.-J. Chen, Y. Mao, M.-Q. Huang, W.-J. Zhang, H. Chen, M. J. Li, D. Nolan, F. Zhou, X. Jiang, Z. Wang, Q. Zhang, X.-B. Wang, and J.-W. Pan, "Measurement- 
device-independent quantum key distribution over a $404 \mathrm{~km}$ optical fiber," Phys. Rev. Lett. 117 (2016).

[34] T. Schmitt-Manderbach, H. Weier, M. Fürst, R. Ursin, F. Tiefenbacher, T. Scheidl, J. Perdigues, Z. Sodnik, C. Kurtsiefer, J. G. Rarity, A. Zeilinger, and H. Weinfurter, "Experimental Demonstration of Free-Space DecoyState Quantum Key Distribution over 144 km," Phys. Rev. Lett. 98, 010504 (2007).

[35] G. Vallone, D. Bacco, D. Dequal, S. Gaiarin, V. Luceri, G. Bianco, and P. Villoresi,"Experimental satellite quantum communications", Phys. Rev. Lett. 115, 040502 (2015).

[36] J.-Y. Wang, B. Yang, S.-K. Liao, L. Zhang, Q. Shen, X.-F. Hu, J.-C. Wu, S.-J. Yang, H. Jiang, Y.-L. Tang, B. Zhong, H. Liang, W.-Y. Liu, Y.-H. Hu, Y.M. Huang, B. Qi, J.-G. Ren, G.-S. Pan, J. Y., J.-J. Jia, Y.-A. Chen, K. Chen, C.-Z. Peng, and J.-W. Pan "Direct and full-scale experimental verifications towards ground-satellite quantum key distribution," Nat. Photon. 7, 387393 (2013).

[37] S.-K. Liao, W.-Q. Cai, W.-Y. Liu, L. Zhang, Y. Li, J.-G. Ren, J. Yin, Q. Shen, Y. Cao, Z.-P. Li, F.-Z. Li, X.-W. Chen, L.-H. Sun, J.-J. Jia, J.-C. Wu, X.-J. Jiang, J.-F. Wang, Y.-M. Huang, Q. Wang, Y.-L. Zhou, L. Deng, T. Xi, L. Ma, T. Hu, Q. Zhang, Y.-A. Chen, N.-L. Liu, X.-B. Wang, Z.-C. Zhu, C.-Y. Lu, R. Shu, C.-Z. Peng, J.-Y. Wang, and J.-W. Pan, "Satellite-to-ground quantum key distribution," Nature 549, 7670 (2017).

[38] P. Eraerds, N. Walenta, M. Legré, N. Gisin, H. Zbinden, “Quantum key distribution and $1 \mathrm{Gbps}$ data encryption over a single fibre," New. J. Phys. 12, 063027 (2010).

[39] Y. Zhao, C.-H. F. Fung, B. Qi, C. Chen, and H.-K. Lo, "Quantum hacking: Experimental demonstration of time-shift attack against practical quantum-key-distribution systems," Phys. Rev. A 78, 042333 (2008).

[40] L. Lydersen, C. Wiechers, C. Wittmann, D. Elser, J. Skaar, V. Makarov, "Hacking commercial quantum cryptography systems by tailored bright illumination," Nat. Photon. 4, 686-689 (2010).

[41] Z. L. Yuan, B. Fröhlich, M. Lucamarini, G. L. Roberts, J. F. Dynes, and A. J. Shields, "Directly phase-modulated light source," Phys. Rev. X 6, 031044 (2016).

[42] K. A. Patel, J. F. Dynes, M. Lucamarini, I. Choi, A. W. Sharpe, Z. L. Yuan, R. V. Penty, and A. J. Shields, "Quantum key distribution for $10 \mathrm{~Gb} / \mathrm{s}$ dense wavelength division multiplexing networks," Appl. Phys. Lett. 104, 051123 (2014).

[43] T. Brougham, C. F. Wildfeuer, S. M. Barnett, and D. J. Gauthier, "The in- 
formation of high-dimensional time-bin encoded photons," Eur. Phys. J. D 70, 214 (2016).

[44] N. J. Cerf, M. Bourennane, A. Karlsson, and N. Gisin, "Security of quantum key distribution using d-level systems," Phys. Rev. Lett. 88, 127902 (2002).

[45] H. Bechmann-Pasquinucci and A. Peres, "Quantum cryptography with 3state systems," Phys. Rev. Lett. 85, 3313-3316 (2000).

[46] H. Bechmann-Pasquinucci and W. Tittel, "Quantum cryptography using larger alphabets," Phys. Rev. A 61, 062308 (2000).

[47] M. Bourennane, A. Karlsson, and G. Björk, "Quantum key distribution using multilevel encoding," Phys. Rev. A 64, 012306 (2001).

[48] M. Mirhosseini, O. S. Magaña-Loaiza, M. N. O'Sullivan, B. Rodenburg, M. Malik, M. P. J. Lavery, M. J. Padgett, D. J. Gauthier, and R. W. Boyd, “Highdimensional quantum cryptography with twisted light," New J. Phys. 17, 033033 (2015).

[49] M. Mafu, A. Dudley, S. Goyal, D. Giovannini, M. McLaren, M. J. Padgett, T. Konrad, F. Petruccione, N. Lütkenhaus, and A. Forbes, "Higherdimensional orbital-angular-momentum-based quantum key distribution with mutually unbiased bases," Phys. Rev. A 88, 032305 (2013).

[50] S. Gröblacher, T. Jennewein, A. Vaziri, G. Weihs, and A. Zeilinger, "Experimental quantum cryptography with qutrits," New J. Phys. 8, 75 (2006).

[51] A. Sit, F. Bouchard, R. Fickler, J. Gagnon-Bischoff, H. Larocque, K. Heshami, D. Elser, C. Peuntinger, K. Günthner, B. Heim, C. Marquardt, G. Leuchs, R. W. Boyd, and E. Karimi, "High-dimensional intracity quantum cryptography with structured photons," optica 4, 1006-1010 (2017).

[52] I. Ali-Khan, C. J. Broadbent, and J. C. Howell, "Large-alphabet quantum key distribution using energy-time entangled bipartite states," Phys. Rev. Lett. 98, 060503 (2007).

[53] T. Zhong, H. Zhou, R. D. Horansky, C. Lee, V. B. Verma, A. E. Lita, A. Restelli, J. C. Bienfang, R. P. Mirin, T. Gerrits, "Photon-efficient quantum key distribution using time-energy entanglement with high-dimensional encoding," New J. Phys. 17, 022002 (2015).

[54] N. T. Islam, C. C. W. Lim, C. Cahall, J. Kim, and D. J. Gauthier, "Provably secure and high-rate quantum key distribution with time-bin qudits," Science advances3, e1701491 (2017).

[55] S. P. Walborn, D. S. Lemelle, M. P. Almeida, and P. H. S. Ribeiro, "Quantum key distribution with higher-order alphabets using spatially encoded qudits," Phys. Rev. Lett. 96, 090501 (2006).

[56] S. P. Walborn, D. S. Lemelle, D. S. Tasca, and P. H. S. Ribeiro, "Schemes 
for quantum key distribution with higher-order alphabets using singlephoton fractional fourier optics," Phys. Rev. A 77, 062323 (2008).

[57] P. B. Dixon, G. A. Howland, J. Schneeloch, and J. C. Howell, "Quantum mutual information capacity for high-dimensional entangled states," Phys. Rev. Lett. 108, 143603 (2012).

[58] S. Etcheverry, G. Cañas, E. S. Gómez, W. A. T. Nogueira, C. Saavedra, G. B. Xavier, and G. Lima, "Quantum key distribution session with 16dimensional photonic states," Sci. Rep. 3, 2316 (2013).

[59] N. Zhao, X. Li, G. Li, and J. M. Kahn, "Capacity limits of spatially multiplexed free-space communication," Nat. Photon. 9, 822-826 (2015).

[60] J. M. Kahn, G. Li, X. Li, and N. Zhao, "To Twist or Not to Twist: Capacity Limits of Free-Space Channels," in "Advanced Photonics 2016 (IPR, NOMA, Sensors, Networks, SPPCom, SOF)" (2016), SpM4E.1

[61] S. A. Goorden, M. Horstnabb, A. P. Mosk, B. Škorić, P. W. H. Pinkse, "Quantum-secure authentication of a physical unclonable key," Optica 1, 421-424 (2014).

[62] R. Pappu, R. Recht, J. Tailor, N. Gershenfeld, "Physical one-way functions," Science 297, 2026-2030 (2002).

[63] R. Maes, Physically Unclonable Functions. (Springer, 2013).

[64] C. Herder, M.-D. Yu, F. Koushanfar, and S. Devadas, “Physical Unclonable Functions and Applications: A Tutorial," Proc. IEEE 102, 1126-1141 (2014).

[65] D. J. C. MacKay, Information theory, inference and learning algorithms (Cambridge University, 2003).

[66] A. Feito, J. S. Lundeen, H. Coldenstrodt-Ronge, J. Eisert, M. B. Plenio, and I. A. Walmsley, "Measuring measurement: theory and practice," New J. Phys. 11, 093038 (2009).

[67] I. D. Ivanovic, "How to differentiate between non-orthogonal states," Phys. Lett. A 123, 257-259 (1987).

[68] D. Dieks, "Overlap and distinguishability of quantum states," Phys. Lett. A 126, 303-306 (1988).

[69] A. Peres, "How to differentiate between non-orthogonal states," Phys. Lett. A 128, 19 (1988).

[70] G. Jaeger and A. Shimony, "Optimal distinction between two nonorthogonal quantum states," Phys. Lett. A 197, 83-87 (1995).

[71] A. Peres and D. R. Terno, "Optimal distinction between non-orthogonal quantum states," J. Phys. A 31, 7105 (1998).

[72] A. Chefles and S. M. Barnett, "Optimum unambiguous discrimination be- 
tween linearly independent symmetric states," Phys. Lett. A 250, 223-229 (1998).

[73] D. Bruß and C. Macchiavello, "Optimal state estimation for d-dimensional quantum systems," Phys. Lett. A 253, 249-251 (1999).

[74] A. S. Holevo, "Bounds for the quantity of information transmitted by a quantum communication channel," Problemy Peredachi Informatsii 9, 311 (1973).

[75] S. Bandyopadhyay, P. O. Boykin, V. Roychowdhury, and F. Vatan, "A new proof for the existence of mutually unbiased bases," Algorithmica 34, 512528 (2002).

[76] C. H. Bennett, G. Brassard, and J.-M. Robert, "Privacy amplification by public discussion," SIAM J. Comput. 17, 210-229 (1988).

[77] D. Deutsch, A. K. Ekert, R. Jozsa, C. Macchiavello, S. Popescu and A. Sanpera, "Quantum privacy amplification and the security of quantum cryptography over noisy channels," Phys. Rev. Lett. 77, 2818 - 2821, (1996). Erratum in ibid 80, 2022, (1998).

[78] T. M. Cover and J. A. Thomas, Elements of information theory 2nd edition, (Wiley-interscience, 2006).

[79] N. Lütkenhaus, "Security against eavesdropping in quantum cryptography," Phys. Rev. A 54, 97-111 (1996).

[80] G. Brassard and L. Salvail, "Lecture notes in computer science," Advances in Cryptology EUROCRYPT'93 765, 410-423 (1994).

[81] J. Martinez-Mateo, C. Pacher, M. Peev, A. Ciurana, and V. Martin,"Demystifying the information reconciliation protocol Cascade," Quantum Inform. Compu. 15, 453-477 (2015).

[82] C. H. Bennett, G. Brassard, C. Crépeau, U. M. Maurer, "Generalized privacy amplification," IEEE Trans. Inf. Theory 41, 1915-1923 (1995).

[83] R. Renner and R. König, "Universally composable privacy amplification against quantum adversaries," Theory of Cryptography Conference, 407425 (2005).

[84] J. L. Carter and M. N. Wegman, "Universal classes of hash functions," J. Comput. Syst. Sci. 18, 143-154 (1979).

[85] G. Lima, L. Neves, R. Guzmán, E. S. Gómez, W. A. T. Nogueira, A. Delgado, A. Vargas, and C. Saavedra, "Experimental quantum tomography of photonic qudits via mutually unbiased basis," Opt. Express 19, 3542-3552 (2011).

[86] I. Csiszár and J. Korner, "Broadcast channels with confidential messages," IEEE Trans. Inf. Theory 24, 339-348 (1978). 
[87] I. Devetak and A. Winter, "Distillation of secret key and entanglement from quantum states," Proc. R. Soc. A 461, 2053, 207-235 (2005).

[88] N. J. Cerf and C. Adami, "Negative entropy and information in quantum mechanics," Phys. Rev. Lett. 79, 5194 (1997).

[89] L. Sheridan, and V. Scarani, "Security proof for quantum key distribution using qudit systems," Phys. Rev. A 82, 030301 (2010).

[90] V. Scarani, and R. Renner, "Quantum cryptography with finite resources: Unconditional security bound for discrete-variable protocols with one-way postprocessing," Phys. Rev. Lett. 100, 200501 (2008).

[91] R. Y. Q. Cai, and V. Scarani, "Finite-key analysis for practical implementations of quantum key distribution," New. J. Phys. 11, 045024 (2009).

[92] H.-K. Lo, H. F. Chau, and M. Ardehali, "Efficient quantum key distribution scheme and a proof of its unconditional security," J. Cryptol. 18, 133-165 (2005).

[93] R. Renner and S. Wolf, "Smooth Rényi entropy and applications," International Symposium on Information Theory, 2004. ISIT 2004. Proceedings. (2004), pp. 233.

[94] V. Makarov and D. R. Hjelme "Faked states attack on quantum cryptosystems," J. Mod. Optic. 52, 691-705 (2005).

[95] W.-Y. Hwang, "Quantum key distribution with high loss: toward global secure communication," Phys. Rev. Lett. 91, 057901 (2003).

[96] W. Heisenberg, “Über den anschaulichen Inhalt der quantentheoretischen Kinematik und Mechanik," Z. Phys. 43, 172-198 (1927).

[97] E. H. Kennard, "Zur quantenmechanik einfacher bewegungstypen," Z. Phys. 44, 326-352 (1927).

[98] D. Korff, "Analysis of a method for obtaining near-diffraction-limited information in the presence of atmospheric turbulence," 2010 Int. Conf. Wirel. Commun. Sens. Comput. 63, 971-980 (1973).

[99] A. A. B. Raj, J. A. V. Selvi, and S. Raghavan, "Terrestrial free space line of sight optical communication (tfslsoc) using adaptive control steering system with laser beam tracking, aligning and positioning (atp)," 2010 Int. Conf. Wirel. Commun. Sens. Comput. 1-5 (2010).

[100] S. Arnon, "Optimization of urban optical wireless communication systems," IEEE Trans. Wirel. Commun. 2, 626-629 (2003).

[101] A. M. Oboukhov, "Some specific features of atmospheric tubulence," J. Fluid Mech. 13, 77-81 (1962).

[102] P. P. Rohde, J. F. Fitzsimons, and A. Gilchrist, “Information capacity of a single photon," Phys. Rev. A 88, 022310 (2013). 
[103] X.-L. Wang, L.-K. Chen, W. Li, H.-L. Huang, C. Liu, C. Chen, Y.-H. Luo, Z.-E. Su, D. Wu, Z.-D. Li, H. Lu, Y. Hu, X. Jiang, C.-Z. Peng, L. Li, N.-L. Liu, Y.-A. Chen, C.-Y. Lu, and J.-W. Pan, "Experimental ten-photon entanglement," Phys. Rev. Lett. 117, 210502 (2016).

[104] J. Wang, J-Y. Yang, I. M. Fazal, N. Ahmed, Y. Yan, H. Huang, Y. Ren, Y. Yue, S. Dolinar, M. Tur, and A. E. Willner, "Terabit free-space data transmission employing orbital angular momentum multiplexing," Nat. Photon. 6, 488496 (2012).

[105] D. J. Gauthier, C. F. Wildfeuer, H. Stipčević, B. Christensen, D. Kumor, P. Kwiat, K. McCusker, T. Brougham, and S. M. Barnett, "Quantum Key Distribution Using Hyperentangled Time-Bin States," in "Proceedings of The Tenth Rochester Conference on Coherence on Quantum Optics (CQO10)," (2014), pp. 234-239.

[106] R. Gallager, "Low-density parity-check codes," IRE Transactions on information theory 8, 21-28 (1962).

[107] F. Gray, "Pulse code communication," (1953). US Patent 2,632,058.

[108] T. Čižmár and K. Dholakia, "Shaping the light transmission through a multimode optical fibre: complex transformation analysis and applications in biophotonics," Opt. Express 19, 18871-18884 (2011).

[109] L. V. Amitonova, A. P. Mosk, and P. W. H. Pinkse, "Rotational memory effect of a multimode fiber," Opt. Express 23, 20569-20575 (2015).

[110] D. J. Lum, J. C. Howell, M. S. Allman, T. Gerrits, V. B. Verma, S. W. Nam, C. Lupo, and S. Lloyd, "Quantum enigma machine: Experimentally demonstrating quantum data locking," Phys. Rev. A 94, 022315 (2016).

[111] D. P. DiVincenzo, M. Horodecki, D. W. Leung, J. A. Smolin, and B. M. Terhal, "Locking classical correlations in quantum states," Phys. Rev. Lett. 92, 067902 (2004).

[112] T. A. W. Wolterink, R. Uppu, G. Ctistis, W. L. Vos, K.-J. Boller, and P. W. H. Pinkse, "Programmable two-photon quantum interference in $10^{3}$ channels in opaque scattering media," Phys. Rev. A 93, 053817 (2016).

[113] B. Jost, A. Sergienko, A. Abouraddy, B. Saleh, and M. Teich, "Spatial correlations of spontaneously down-converted photon pairs detected with a single-photon-sensitive ccd camera," Opt. Express 3, 81-88 (1998).

[114] A. F. Abouraddy, M. B. Nasr, B. E. A. Saleh, A. V. Sergienko, and M. C. Teich, "Demonstration of the complementarity of one-and two-photon interference," Phys. Rev. A 63, 063803 (2001).

[115] O. Jedrkiewicz, Y.-K. Jiang, E. Brambilla, A. Gatti, M. Bache, L. Lugiato, and P. Di Trapani, "Detection of sub-shot-noise spatial correlation in highgain parametric down conversion," Phys. Rev. Lett. 93, 243601 (2004). 
[116] O. Haderka, J. Peřina Jr, M. Hamar, and J. Peřina, “Direct measurement and reconstruction of nonclassical features of twin beams generated in spontaneous parametric down-conversion," Phys. Rev. A 71, 033815 (2005).

[117] H. D. L. Pires, C. H. Monken, and M. P. van Exter, "Direct measurement of transverse-mode entanglement in two-photon states," Phys. Rev. A 80, 022307 (2009).

[118] R. S. Aspden, D. S. Tasca, R. W. Boyd, and M. J. Padgett, "Epr-based ghost imaging using a single-photon-sensitive camera," New J. Phys. 15, 073032 (2013).

[119] R. Fickler, M. Krenn, R. Lapkiewicz, S. Ramelow, and A. Zeilinger, "Realtime imaging of quantum entanglement," Sci. Rep. 3, 1914 (2013).

[120] R. Chrapkiewicz, W. Wasilewski, and K. Banaszek, "High-fidelity resolved multiphoton counting for quantum imaging applications," Opt. Lett. 39, 5090-5093 (2014).

[121] I. M. Vellekoop and A. P. Mosk, “Focusing coherent light through opaque strongly scattering media," Opt. Lett. 32, 2309-2311 (2007).

[122] R. Barends, A. Shabani, L. Lamata, J. Kelly, A. Mezzacapo, U. Las Heras, R. Babbush, A. G. Fowler, B. Campbell, Y. Chen, Z. Chen, B. Chiaro, A. Dunsworth, E. Jeffrey, E. Lucero, A. Megrant, J. Y. Mutus, M. Neeley, C. Neill, P. J. J. O'Malley, C. Quintana, P. Roushan, D. Sank, A. Vainsencher, J. Wenner, T. C. White, E. Solano, H. Neven, and J. M. Martinis, "Digitized adiabatic quantum computing with a superconducting circuit," Nature 534, 222 (2016).

[123] T. Brecht, W. Pfaff, C. Wang, Y. Chu, L. Frunzio, M. H. Devoret, and R. J. Schoelkopf, "Multilayer microwave integrated quantum circuits for scalable quantum computing," npj Quantum Inf. 2, 16002 (2016).

[124] D. Aasen, M. Hell, R. V. Mishmash, A. Higginbotham, J. Danon, M. Leijnse, T. S. Jespersen, J. A. Folk, C. M. Marcus, K. Flensberg, and J. Alicea, "Milestones toward Majorana-based quantum computing," Phys. Rev. X 6, 031016 (2016).

[125] M. Saffman, "Quantum computing with atomic qubits and Rydberg interactions: progress and challenges," J. Phys. B 49, 202001 (2016).

[126] Y. Wang, X. Li, Z.-Q. Yin, and B. Zeng, "16-qubit IBM universal quantum computer can be fully entangled," preprint arXiv:1801.03782 (2018).

[127] A. J. Menezes, P. C. Van Oorschot, and S. A. Vanstone, Handbook of applied cryptography (CRC press, 1996).

[128] I. Ali-Khan, C. J. Broadbent, and J. C. Howell, "Large-alphabet quantum key distribution using energy-time entangled bipartite states," Phys. Rev. Lett. 98, 060503 (2007). 
[129] J. Nunn, L. J. Wright, C. Söller, L. Zhang, I. A. Walmsley, B. J. Smith, "Large-alphabet time-frequency entangled quantum key distribution by means of time-to-frequency conversion," Opt. Express 21, 15959-15973 (2013).

[130] M. Krenn, M. Huber, R. Fickler, R. Lapkiewicz, S. Ramelow, and A. Zeilinger, "Generation and confirmation of a $(100 \times 100)$-dimensional entangled quantum system," Proc. Natl. Acad. Sci. U.S.A. 111, 6243-6247 (2014).

[131] N. Zhao, X. Li, G. Li, J. M. Kahn, “Capacity limits of spatially multiplexed free-space communication," Nat. Photon. 9, 822-826 (2015).

[132] J. Kahn, G. Li, X. Li, and N. Zhao, "To Twist or Not to Twist: Capacity Limits for Free-Space Channels," in Advanced Photonics 2016 (IPR, NOMA, Sensors, Networks, SPPCom, SOF), OSA Technical Digest (online) (Optical Society of America, 2016), paper SpM4E.1.

[133] I. M. Vellekoop and A. P. Mosk, "Focusing coherent light through opaque strongly scattering media," Opt. Lett. 32, 2309-2311 (2007).

[134] T. B. H. Tentrup, T. Hummel, T. A. W. Wolterink, R. Uppu, A. P. Mosk, and P. W. H. Pinkse, "Transmitting more than 10 bit with a single photon," Opt. Express 25, 2826-2833 (2017).

[135] M. Tomamichel, J. Martinez-Mateo, C. Pacher, and D. Elkouss, "Fundamental finite key limits for information reconciliation in quantum key distribution," 2014 IEEE International Symposium on Information Theory, Honolulu, HI, 2014, pp. 1469-1473.

[136] G. Gilbert, M. Hamrick, and F. J. Thayer, "Privacy Amplification in Quantum Key Distribution: Pointwise Bound versus Average Bound," preprint quant-ph/0108013 (2001).

[137] L. V. Amitonova, T. B. H. Tentrup, I. M. Vellekoop, and P. W. H. Pinkse, "Multimode-fiber-based high-dimensional quantum secure communication," preprint arXiv:1801.07180 (2018).

[138] H.-K. Lo, M. Curty, and K. Tamaki, "Secure quantum key distribution," Nat. Photon. 8, 595--604 (2014).

[139] G.-L. Long and X.-S. Liu, "Theoretically efficient high-capacity quantumkey-distribution scheme," Phys. Rev. A 65, (2002).

[140] K. Boström and T. Felbinger, "Deterministic Secure Direct Communication Using Entanglement," Phys. Rev. Lett. 89, 187902 (2002).

[141] F-G. Deng, G. L. Long, and X-S. Liu, "Two-step quantum direct communication protocol using the Einstein-Podolsky-Rosen pair block," Phys. Rev. A 68, 042317 (2003).

[142] F.-G. Deng and G. L. Long, "Secure direct communication with a quantum one-time pad," Phys. Rev. A 69, 052319 (2004). 
[143] C. Wang, F.-G. Deng, Y.-S. Li, X.-S. Liu, and G. L. Long, “Quantum secure direct communication with high-dimension quantum superdense coding," Phys. Rev. A 71, 044305 (2005).

[144] J.-Y. Hu, B. Yu, M.Y. Jing, L.-T. Xiao, S.-T. Jia, G.-Q. Qin, G.-L Long, “Experimental quantum secure direct communication with single photons," Light Sci. Appl. 5, e16144 (2016).

[145] W. Zhang, D.-S Ding, Y.-B. Sheng, L. Zhou, B.-S. Shi, G.-C. Guo, “Quantum Secure Direct Communication with Quantum Memory," Phys. Rev. Lett. 118, 220501 (2017).

[146] J. G. Proakis, Wiley Encyclopedia of Telecommunications. (Wiley-Interscience, 2003).

[147] M. Plöschner, T. Tyc, and T. Čižmár, "Seeing through chaos in multimode fibres," Nat. Photon. 9, 529-535 (2015).

[148] A. W. Snyder and J. Love, Optical waveguide theory. (Springer Science \& Business Media, 1983).

[149] Y. Bromberg, B. Redding, S. M. Popoff, and H. Cao, "Remote key establishment by mode mixing in multimode fibres and optical reciprocity," preprint arXiv:1506.07892 (2015).

[150] I. M. Vellekoop, A. Lagendijk, and A. P. Mosk, "Exploiting disorder for perfect focusing," Nat. Photon. 4, 320 (2010).

[151] J. Jang, J. Lim, H. Yu, H. Choi, J. Ha, J.-H. Park, W.-Y. Oh, W. Jang, S. Lee, and Y. Park, "Complex wavefront shaping for optimal depth-selective focusing in optical coherence tomography," Opt. Express 21, 2890-2902 (2013).

[152] T. Čižmár and K. Dholakia, "Shaping the light transmission through a multimode optical fibre: complex transformation analysis and applications in biophotonics," Opt. Express 19, 18871-18884 (2011).

[153] T. Čižmár and K. Dholakia, "Exploiting multimode waveguides for pure fibre-based imaging," Nat. Commun. 3, 1027 (2012).

[154] R. Di Leonardo and S. Bianchi, "Hologram transmission through multimode optical fibers," Opt. Express 19, 247--254 (2011).

[155] L. V. Amitonova, A. P. Mosk, and P. W. H. Pinkse, "The rotational memory effect of a multimode fiber," Opt. Express 23, 20569 (2015).

[156] L. V. Amitonova, A. Descloux, J. Petschulat, M. H. Frosz, G. Ahmed, F. Babic, X. Jiang, A. P. Mosk, P. St J. Russell, and P. W. H. Pinkse, "Highresolution wavefront shaping with a photonic crystal fiber for multimode fiber imaging," Opt. Lett. 41, 497-500 (2016).

[157] A. Descloux, L. V. Amitonova, and P. W. H. Pinkse, "Aberrations of the 
point spread function of a multimode fiber due to partial mode excitation," Opt. Express 24, 18501-18512 (2016).

[158] I. M. Vellekoop, "Feedback-based wavefront shaping," Opt. Express 23, 12189-12206 (2015).

[159] I. M. Vellekoop, A. P. Mosk, “Universal Optimal Transmission of Light Through Disordered Materials," Phys. Rev. Lett. 101, 120601 (2008).

[160] M. Jang, C. Yang, and I. M. Vellekoop, "Optical Phase Conjugation with Less Than a Photon per Degree of Freedom," Phys. Rev. Lett. 118, 093902 (2017).

[161] I. N. Papadopoulos, S. Farahi, C. Moser, and D. Psaltis, "Increasing the imaging capabilities of multimode fibers by exploiting the properties of highly scattering media," Opt. Lett. 38, 2776--2778 (2013).

[162] Y. Choi, C. Yoon, M. Kim, J. Yang, and W. Choi, "Disorder-mediated enhancement of fiber numerical aperture," Opt. Lett. 38, 2253-2255 (2013).

[163] S. M. Kim, T. T. Do, T. J. Oechtering, and G. Peters, “On the Entropy Computation of Large Complex Gaussian Mixture Distributions," IEEE Trans. Signal Process. 63, 4710--4723 (2015).

[164] B. Škorić, "Quantum Readout of Physical Unclonable Functions," Int. J. Q. Inf. 10, 1250001 (2012).

[165] B. Škorić, A. P. Mosk, P. W. H. Pinkse, "Security of quantum-readout PUFs against quadrature-based challenge-estimation attacks," Int. J. Q. Inf. 11, 135401 (2013).

[166] B. Škorić, "Security analysis of Quantum-Readout PUFs in the case of challenge-estimation attacks," Quant. Inf. Comp. 16, 0050-0060 (2016).

[167] R. Uppu, T. W. A. Wolterink, S. A. Goorden, B. Chen, B. Škorić, A. P. Mosk, and P. W. H. Pinkse, "Asymmetric Cryptography with Physical Unclonable Keys," preprint arXiv:1802.07573 (2018).

[168] B. Javidi, et al. "Roadmap on optical security," J. Opt. 18, 083001 (2016).

[169] B. Škorić, P. W. H. Pinkse, and A. P. Mosk, "Authenticated communication from Quantum Readout of PUFs," Quantum Information Processing 16, 200 (2017).

[170] C. H. Bennett, G. Brassard, and S. Breidbart, "Quantum cryptography II: How to re-use a one-time pad safely even if $\mathrm{P}=\mathrm{NP}$," Nat. Comput. 13, 453-458 (2014).

[171] B. Škorić and M. de Vries, "Quantum key recycling with 8-state encoding (The quantum one-time pad is more interesting than we thought)," Int. J. Q. Inf. 15, 1750016 (2017).

[172] U. Vazirani and T. Vidick, "Fully Device-Independent Quantum Key Dis- 
tribution," Phys. Rev. Lett. 113, 140501 (2014); Erratum Phys. Rev. Lett. 116, 089901 (2016).

[173] T. Sasaki, Y. Yamamoto, and M. Koashi, "Practical quantum key distribution protocol without monitoring signal disturbance," Nature 509, 475-478 (2014).

[174] M. S. Allman, V. B. Verma, M. Stevens, T. Gerrits, R. D. Horansky, A. E. Lita, F. Marsili, A. Beyer, M. D. Shaw, D. Kumor, R. Mirin, and S. W. Nam, "A near-infrared 64-pixel superconducting nanowire single photon detector array with integrated multiplexed readout," Appl. Phys. Lett. 106, 192601 (2015).

[175] P. K. Day, H. G. LeDuc, B. A. Mazin, A. Vayonakis, and J. Zmuidzinas, "A broadband superconducting detector suitable for use in large arrays," Nature 425, 6960 (2003).

[176] M. Malik, M. O'Sullivan, B. Rodenburg, M. Mirhosseini, J. Leach, M. P. J. Lavery, M. J. Padgett, Miles, and R. J. Boyd, "Influence of atmospheric turbulence on optical communications using orbital angular momentum for encoding," Opt. express 20, 13195-13200 (2012). 



\section{Samenvatting}

In dit proefschrift worden methoden voor kwantumsleuteldistributie (Engelse afkorting: QKD) en kwantumcommunicatie ontwikkeld, die gebaseerd zijn op het hoog-dimensionaal, ruimtelijk coderen van licht. Deze methoden danken hun veiligheid aan de fundamentele kwantumeigenschappen van licht. De veiligheid wordt versterkt door de hoog-dimensionale Hilbertruimtes die verbonden zijn aan afbeeldingsoptiek. Het proefschrift begint met een overzicht van klassieke informatietheorie en kwantuminformatie. Dit overzicht dient als inleiding tot de kwantumcryptografie. Vervolgens worden de voordelen van hogerdimensionale alfabetten, en de veiligheid van het standaard BB84 protocol met twee bases, aangetoond. De ruimtelijke toestanden die gebruikt worden voor codering worden geïntroduceerd. Er wordt een analytische uitdrukking afgeleid voor de bovengrens voor de wederzijdse informatie (mutual information). Hierbij wordt rekening gehouden met multi-foton toestanden, detectorruis en bundelverbreding. Het hoog-dimensionaal coderen van enkele fotonen wordt experimenteel aangetoond. Hierbij is 10.5 bit per ontvangen foton behaald. Er wordt besproken hoe de wederzijdse informatie afhangt van het aantal pixels van de detector. Daarnaast worden de experimenteel behaalde waardes vergeleken met de verwachte bovengrens op basis van theorie. Er is aangetoond dat een standaard LDPC code voor foutcorrectie volstaat om vrijwel foutloze communicatie te behalen. Er is, door een tweede basis toe te voegen, een groot-alfabet QKD systeem gerealiseerd en gekarakteriseerd. Er wordt geanalyseerd hoe veilig dit BB84-achtige protocol is tegen intercept-resend- en globale aanvallen. De sleutelgeneratie-frequentie na nabewerking wordt ook geanalyseerd. Hierbij wordt uitgegaan van een realistische situatie, met sleutels van eindige lengte. Ten slotte wordt er een nieuwe methode voor kwantumcommunicatie gedemonstreerd, waarbij informatie gecodeerd wordt in golffronten die verdeeld zijn over de modes van een multimode glasvezel. Aan het einde van het proefschrift worden verschillende methoden voor kwantumversleuteling, kwantumcryptografie en Quantum Data Locking met elkaar vergeleken. Hierbij wordt gekeken naar de karakteristieken van het klassieke kanaal, het kwantumkanaal en de dimensie van de Hilbertruimte. 



\section{Acknowledgements}

This thesis as the culmination of my PhD could not have been written without the help and support of many persons I am proud and thankful to honor in this section.

First, I would like to thank my mentor and supervisor Pepijn Pinkse for his help, support and training during the last four years. He did not hesitate to let an avowed theorist in his lab. I appreciate his patience with my timid questions in the beginning of the $\mathrm{PhD}$, e.g., if there are special courses on experimental lab work I could follow to find out what all the shiny equipment is for. His enthusiasm in research pushed me further and I am appreciative for his training in experimental physics and scientific writing. I admire his capability to remain calm in difficult situations and it was a pleasure to have had him as a guest at my wedding.

I am grateful towards Marc Bienert and Giovanna Morigi for their excellent training in theoretical physics. They laid the foundation for my work as a scientist and sparked my passion for quantum optics.

I thank Dirk Bouwmeester, Hans Hilgenkamp, Ad Lagendijk and Christoph Marquardt for carefully reading my thesis and for being committee members in my defense.

I experienced the COPS group as an inspiring and motivating environment for research. This environment is mainly shaped by the seniors Willem Vos, Pepijn Pinkse, Allard Mosk and Ad Lagendijk. Willem never failed to provide pointed feedback on my presentations, which helped me to improve. I thank Allard for the useful discussions and sharing his immense knowledge about several topics. Ad, thank you for always pointing out good scientific behavior and ensuring high scientific standards. I am grateful for meeting Rebecca Saive and Jan Klärs. Although our time at COPS only overlapped for a few months, they gave inspiring new stimuli. My special thanks also goes to the secretaries I met over the years who kept the group together through their hard work. These are Nilda, Jacqueline, Carla, Nienke and Nicole. Especially Nicole for always having time for any small question and for all her help in organizing my defense. I thank Cock Harteveld for managing a great lab and Marlon Gomperts for his help in ICTS matters. Many thanks to all the COPS colleagues I worked with: I appreciate having worked with the great colleagues Tom Wolterink, Sergei Sokolov, Devashish, Andreas Schulz, Hasan Yilmaz, Bas Goorden, Jin Lian, Femi Ojambai, Diana Grishina, Maryna Meretska, Sjoerd Hack, Manashee Adhikary, Reinier van der Meer and Evangelos Marakis. I thank the postdocs Emre Yüce, Georgios Ctistis, Henri Nielsen, Lyuba Amitonova, Shakeeb Bin Hasam, Ravitej Uppu, Mehdi Aas and Jelmer Renema for the fruitful discussions and their kind help. I praise Tom for all the help in the lab. I am deeply impressed by his skill to let every experimental problem disappear. My greatest respect goes to Ravi for being a walking encyclopedia and for his optimism to always fit another optical element in. I thank Sjoerd for his advice concerning pool safety. Diana and 
Maryna earned my appreciation for initiating all the fun group activities and shaping the group spirit. Manashee, bless your kind soul and twisted humor. Devashish remains in a special position for being a great office buddy.

I had the pleasure to make acquaintance with the students Matthijs, Jeroen, Klaas-Jan, Maarten, Frank, Roland, Chris and the exchange students Jorge, Julio and Vincent, who I gratefully remember for the vivid conversations during the coffee breaks. I thank Peter Hooijschuur and Reinier van der Meer for starting off the project on Large-alphabet QKD in their Bachelor project. The collaboration with Ivo Vellekoop and Lyuba Amitonova resulted in chapter 6. I appreciated the discussions about security proofs and multimode fibers.

My time at COPS also was the start of many close friendships. Here, I want to highlight Ravitej Uppu and Evangelos Marakis, who I shared the longest time in the AQO group with and who kindly agreed on being my paranymphs. Thank you for your enthusiasm towards Mango Chicken and Skepasti, for long talks or short jokes, coffee and smoking breaks.

During my PhD, I had the pleasure to work closely with several Bachelor and Master students. Mentoring these students allowed me to learn a lot myself. The first to name here is Thomas Hummel, my first student. The discussions and work with him resulted in chapter 3 of this thesis. He impressed us with his "Dutch" humor and his fearless work with high voltages. I thank my second bachelor student Willemijn Luiten for the work which helped to design the experimental setup in chapter 5. Last but not least, I thank Chris Toebes and Ben Kassenberg for all the fruitful (and humorous) discussions during their Bachelor projects.

I applaud Jesse Mak for waking my passion in tennis again. I thank him for being my double partner, finally leading to winning the campus tennis competition. I very much appreciate his help in translating the summary of this thesis.

The members of our neighboring group LPNO also gave a helping hand: I thank the group leader Klaus Boller for his suggestions, and Peter van der Slot and Bert Bastiaens for help with the lab equipment. I have greatly benefited from the fruitful discussions with Jörn Epping, Caterina Taballione, Jesse Mak, Youwen Fan, Marco Garcia Porcel and Yin Tao. A special thanks goes to Yin Tao and Tom Wolterink for their help with my cover image.

I would also like to express my thanks to the MESA+ Institute for Nanotechnology for offering a great environment for research. The ANP group meetings provided an excellent atmosphere for scientific exchange, I feel blessed to have been a part of them. My deepest respect and gratefulness is directed to the EU for their ongoing work in overcoming borders, of utmost importance in supporting research collaborations across Europe.

I thank my wife Judith Hoth for her support and help. She showed great patience with my carried-home research questions. Without her, this thesis would have been impossible. Thank you for always believing in me and for celebrating 
our wonderful wedding in the final year of my PhD. I also thank her parents Sebastian Hoth and Margit Hoth for welcoming me in this family. They were always ready to challenge me with questions about quantum phenomena.

I am indebted to my parents Ilka Eisenbeis-Tentrup and Thomas Tentrup for all their support not only during my PhD, but also during and before my entire student carrier. My brother Leander Tentrup and my aunt Anke Eisenbeis never failed to express their support or to provide a welcome side plot. My warmest thanks are reserved for my grandparents Ellen Kurz, Mechthild und Horst Eisenbeis. Especially Mechthild and Horst, who cannot be thanked enough for always having been there for me and my brother. 